Portland State University

PDXScholar

Spring 10-1-2013

\title{
Bringing Functional Family Probation Services to the Community: A Qualitative Case Study
}

Denise Lynmarie Austin

Portland State University

Follow this and additional works at: https://pdxscholar.library.pdx.edu/open_access_etds

Part of the Counseling Commons, Criminology and Criminal Justice Commons, and the Marriage and Family Therapy and Counseling Commons

Let us know how access to this document benefits you.

\section{Recommended Citation}

Austin, Denise Lynmarie, "Bringing Functional Family Probation Services to the Community: A Qualitative Case Study" (2013). Dissertations and Theses. Paper 1436.

https://doi.org/10.15760/etd.1435

This Thesis is brought to you for free and open access. It has been accepted for inclusion in Dissertations and Theses by an authorized administrator of PDXScholar. Please contact us if we can make this document more accessible: pdxscholar@pdx.edu. 
Bringing Functional Family Probation Services to the Community:

A Qualitative Case Study

by

Denise Lynmarie Austin

A thesis submitted in partial fulfillment of the requirements for the degree of

Master of Science

in

Criminology and Criminal Justice

Thesis Committee:

William Feyerherm, Chair

Danielle McGurrin

Brian Renauer

Charlene Rhyne

Portland State University

2013 
C 2013 Denise Lynmarie Austin 


\begin{abstract}
In March 2011, Multnomah County's Juvenile Services Division (JSD) in Portland, Oregon implemented a new program model called Functional Family Probation Services, a case management model based on the principles of Functional Family Therapy. Under this model JSD Juvenile Court Counselors deliver Functional Family Probation Services to medium and high-risk youth on probation; both to the youth and their family in their home. This qualitative case study examined the extent to which the Juvenile Court Counselors and Community Justice Managers implemented Functional Family Probation Service's components and recorded their opinions regarding Functional Family Probation Services as a case management model. The study included observations of consultation meetings and interviews of 17 Juvenile Court Counselors, Community Justice Managers, and Senior Managers. The primary results of the study revealed that the Functional Family Probation Services intervention is well received by the county's youth and families and that Functional Family Probation Services increases family functioning. However, both observations and self-reports from the Juvenile Court Counselors and Community Justice Managers indicated that Functional Family Probation Services requires individual and organizational changes critical for its successful implementation and sustainability.
\end{abstract}




\section{Dedication}

This thesis is dedicated

to Multnomah County's Youth and Families 


\section{Acknowledgments}

This thesis resulted from the support of Multnomah County Department of Community Justice Juvenile Services Division. I want to thank Dr. Charlene Rhyne and Lailah Hamblin, M.S. of the Quality Control, Research \& Evaluation Department of Multnomah County Department of Community Justice for their constant professional and emotional support. In addition, I want to acknowledge the members of my committee: Dr. William Feyerherm, my Chair, Dr. Brian Renauer, Dr. Danielle McGurrin, and Dr. Charlene Rhyne. I would also like to thank Dr. David Morgan and Dr. Peter Collier for providing guidance and feedback on qualitative research methods and design during this study. In addition I'd like to thank Karen Cellarius, M.S. and Dr. Debbie Elliot of Portland State University's Regional Research Institute on their ongoing advice and support on the realities of program evaluation. I also like to thank Dr. Daveena Tauber for reading portions of this thesis and providing me feedback on the format and content.

Further, I am indebted to Christina McMahan, the Assistant Director of Juvenile Services Division, in allowing me to conduct my research at Multnomah County Juvenile Services Division. Finally, I would like to thank all the Juvenile Court Counselors, the Community Justice Managers, and the Senior Managers who participated in this study. Without their support and kindness this study would not have been achieved. 


\section{Table of Contents}

$\begin{array}{lr}\text { Abstract } & \text { i } \\ \text { Dedication } & \text { ii } \\ \text { Acknowledgments } & \text { iii } \\ \text { Chapter One } & 1 \\ \text { Chapter Two } & 40 \\ \text { Chapter Three } & 76 \\ \text { Chapter Four } & 93 \\ \text { Chapter Five } & 131 \\ \text { References } & 145 \\ \text { Appendices } & \end{array}$

A. Juvenile Court Counselor Interview Questions 164

B. Community Justice Manager Interview Questions 166

C. Senior Management Interview Questions 167

D. FFPS Consultation Meeting Observation Checklist 168

E. Human Subjects Review Letter 170 


\section{CHAPTER ONE}

\section{Introduction}

With increasing interest in introducing more effective community supervision programs Multnomah County Juvenile Services Division (JSD) in March 2011, implemented Functional Family Probation Services (FFPS), a case management model based on the evidence treatment Functional Family Therapy (FFT). Under this model JSD Juvenile Court Counselors deliver FFPS to medium and high-risk youth on probation to the youth and family in their home. JSD decision makers elected to implement FFPS after the State of Washington's Juvenile Rehabilitation Administration evaluation indicated that parole officers can be trained to deliver FFPS and that FFPS is cost effective in reducing recidivism (Juvenile Rehabilitation Administration [JRA], 2008; 2012). To date, other juvenile justice agencies that have implemented FFPS and FFT include Los Angeles and Yolo County, California; Atlanta, Georgia; Idaho; and the State of Washington (Casey Family Programs, 2010; Gordon \& Arbuthnot, 1988; Juvenile Rehabilitation Administration [JRA], 2008; Multnomah County Department of Community Justice, 2010). However, only the state of Washington has rigorously evaluated its juvenile parole officer model adherence and competence to determine Functional Family Probation Service's efficacy in reducing recidivism. Those evaluation results suggest that as long as quality assurance protocols and monitoring maintain a parole officer's model adherence and competence, FFPS is cost-effective in reducing 
recidivism (Barnoski, 2004; JRA, 2008, 2012; Rowland, 2007; Sexton, 2011).

In 2003, the Oregon State Legislature passed Senate Bill 267. Senate Bill 267 requires Oregon's agencies to use interventions based on evidence-based practices throughout the Oregon Department of Corrections, Oregon Youth Authority, Oregon Commission on Children and Families, Department of Human Services, and the Oregon Criminal Justice Commission (Latessa, 2004). Because Oregon was moving toward evidence-based practices, JSD leadership began searching for rehabilitative models based on evidence-based principles that would reduce recidivism and better serve the county"s youth and families. Many evidence-based rehabilitative programs designed to serve juvenile delinquents and their families are family-based interventions, such as FFT and Multisystemic Therapy (MST) (Alexander \& Parsons, 1973; Gordon, Graves, \& Arbuthnot, 1995; Huey, Henggeler, Brondino, \& Pickrel, 2000; Sexton \& Alexander, 2002; 2003, Sexton, 2011).

Selected Juvenile Court Counselors and Senior Managers met with juvenile parole leadership in the State of Washington and in the State of Idaho to investigate those agencies' experiences with FFPS and FFT as an intervention used by juvenile parole and probation officers. After a series of meetings with both agencies, JSD leadership decided that FFPS, a case management model designed for community corrections professionals based on FFT, would fit JSD's mission in applying rehabilitative approaches to the 
county's youth and families (D. Corso; Hamblin, L. personal communication, August 12, 2012; July 10, 2012).

Research suggests that implementation of family community-based models involve multi-year training, frequent consultations, and monitoring of corrections professionals to ensure a counselors model adherence and competence (Alexander, et al., 1998, Barnoski, 2002b; 2004, 2005; Henggeler, Melton, Brondino, Scherer, D., \& Hanley, 1997; Henggeler, Pickrel, \& Brondino, 1999). In fact, a growing body of literature on family-based interventions, such as FFT and MST suggest that a counselor's competence moderates increased program outcomes and family functioning (Henggeler, et al., 1997; Huey, et al., 2000). However, evaluation studies frequently conclude that most agencies attracted to community-based programs lack the organizational culture and will to maintain effective monitoring and quality assurance protocols designed to sustain the intervention's effectiveness (Latessa, 2004).

Translating evidence-based community models are challenging to implement; thus, it is crucial that the intervention matches the mission and culture of the organization to ensure successful implementation and sustainability of the intervention (Rhine, Mawhorr, \& Parks, 2006; Zazzalli et al., 2008). Maintaining effective monitoring and quality assurance requires strong organizational support and resources at all levels of an organization to ensure successful implementation of all program components (Latessa, 2004; Mihalec, Irwin, Fagan, Ballard, \& Elliott, 2004). Although program fidelity is 
rarely perfect, Backer (2002) warns that agencies not ready for a community-based intervention tend to adjust the model by making deletions and additions of program components, which include changing the manner and intensity, as well as the dosage (amount of time per session). Moreover, Schoenwald and Hoagwood (2001) and Rhine, Mawhorr, and Parks (2006) state that if there is a lack of fit, organizations may need to change aspects of their organization to provide more resources or alter or adapt aspects of the organization to foster better delivery of the model. Thus, it is important that frequent evaluations and researchers and practitioners work together to confront the myriad of inevitable organizational barriers and hurdles to successfully implement programs without compromising program integrity (Latessa \& Holsinger, 1998; Rhine, et al., 2006).

An agency must examine its organizational readiness and context on its ability to maintain effective monitoring and quality assurance protocols in order to maintain program fidelity (Latessa, 2004). Only recently studies have attributed organizational and individual-level factors that play a critical role in effective implementation of community-based interventions (Fagan \& Mihalic, 2003; Glisson, 2002; Schoenwald \& Hoagwood, 2001). Organizational features and individual-level factors that may preclude fidelity during implementation include resistance, lack of technology, the lack of culture and climate, and the lack of belief (buy-in) among staff (Zazalli et al., 2008; Hemmelgarn, Glisson, \& James, 2006; Joplin et a., 2004; Petersilia, 1990). On the other 
hand, agencies that possess strong leaders, have the technology, and support an evidenced-based culture has the ability to ensure effective delivery and monitoring of core components (Joplin et al., 2004; Latessa, 2004; Latessa \& Holsinger, 1998). Nevertheless, correctional agencies have had limited success because of an agencies failure to change or in part of the many political obstacles inherent to corrections agencies (Latessa, 2004, Rhine et al., 2006).

Frequent assessments of organizational and individual factors that may preclude program fidelity provides critical information for agencies to inform change during implementation (Fixsen, Naoom, Blasé, Friedman, \& Wallace, 2005; Latessa \& Holsinger, 1998; Lowenkam \& Latessa, 2005; Lowenkamp, Latessa, \& Smith, 2006; Mihalic et al., 2004; Zazzali et al., 2008). Unfortunately, most evaluators have focused only on outcome studies, and as a result, have not provided a discussion on how to implement programs and the organizational changes critical during implementation (Fagan, 1990; Latessa, 2004). Practitioners know what to implement, but know very little on how to implement (Mihalic \& Irwin, 2003). Practitioners frequently struggle with implementation and to increase staff buy-in they frequently succumb to changing the components, delivery modes, and dosages of a model (Elliott \& Mihalic, 2004).

Elliot and Mihalic (2004) stress that changing components to increase buy-in is likely counterproductive, for relatively few prevention programs have conducted studies determining the mediating effects of program outcomes to verify which components can 
be deleted or modified. Furthermore, agency leadership must keep in mind that success with a community-based model in one jurisdiction may not have similar successes in another jurisdiction, for programs can be difficult to replicate (Mihalec \& Irwin, 2003; Zazalli et al., 2008). For instance, the study conducted in the State of Washington on the juvenile parole officers delivering FFPS suggest positive outcomes from highly adherent officers may not have similar successes at another agency.

To date only two studies have examined community corrections professionals' model adherence and competence delivering FFT and FFPS. The first study examined the model adherence and competence of juvenile parole officers in the State of Washington who delivered FFPS (Rowland, 2007) and the second study examined Juvenile Probation Officers during their training and delivery of FFT in Atlanta, Georgia (Gordon \& Arbuthnot, 1988). Gordon \& Arbuthnot's (1988) study in Atlanta, Georgia supported the notion that Juvenile Probation Officers could be trained to deliver FFT and the authors identified the organizational and individual-level factors that assist and preclude program delivery. Factors that facilitated the implementation of FFT included appropriate selection of trainees, detailed training methodology, and frequent consultations and booster workshops Factors that the Juvenile Probation Officers struggled with in Atlanta included the lack of detailed training sessions and the inability to prioritize the workload and resources dedicated to the frequent delivery and monitoring of FFT. Frequently, FFT requirements were set aside because of other Juvenile Probation Officers' duties. 
Moreover, Juvenile Probation Officer managers did not believe in the model and did not allow the Juvenile Probation Officers to dedicate the required amount of time and dosage recommended by the FFT manual. Because of those limitations, FFT in Atlanta was terminated (Gordon \& Arbuthnot, 1988).

The second study examined juvenile parole officers' model adherence and competence of FFPS and youth recidivism in all 14 counties in the State of Washington (Rowland, 2007). Rowland (2007) evaluated the recidivism of youth who received the intervention $(\mathrm{n}=621)$ and compared them to a matched control group who received traditional parole services $(\mathrm{n}=621)$. Model adherence and competence of juvenile parole officers delivering FFPS was recorded in the Global Rating Measure (GRM) created by FFT, Inc. The Global Rating Measure is a 34-item observational instrument for quantifying a counselor's level of adherence to the fundamental clinical components of the FFPS model developed by Alexander, Kopp, \& Sexton (2002) (as cited in Rowland, 2007). Supervisors use the GRM to rate the performance of juvenile parole officers and studies suggest that supervisor ratings of therapists' adherence to the FFT model have been linked to behavioral outcomes (Barnoski, 2002; 2004). The GRM ratings in Rowland's (2007) study showed that juvenile parole officers adhered to the principles and skills of each of the phases of the model in the "fairly well" to "well" ranges, meaning that the parole counselors were adherent to the phase-specific skills $25-75 \%$ of the time. Rowland's (2007) results suggested that the combination of juvenile parole officers' 
adherence to the model and a measure of the youth's risk to re-offense (crime severity index) explained a significant portion of the variance in post-parole criminal severity among the treatment group. Logistic regression results, although not statistically significant, demonstrated a $14.6 \%$ reduction in 12 -month felony recidivism rates for those youth who received the FFPS intervention from highly-adherent parole counselors in comparison with those youth receiving traditional parole services. Overall, the implications of this study revealed that juvenile parole officers could be trained to deliver the model and that as the parole counselor's adherence to the FFPS model increased, there was a significant decrease in a youth's post-parole criminal severity (Rowland, 2007). Moreover, Rowland's (2007) findings, similar to findings found in studies examining family therapists delivering FFT and MST, monitoring and quality assurance protocols are critical for FFPS's effectiveness in reducing recidivism and increasing family functioning (Barnoski, 2002, 2004; Henggeler et al., 1997; Rowland, 2007). However, despite reports and studies that suggest FFPS's promise in Washington, politics and budgetary reductions pose challenges to program fidelity. A report to the state legislature from the state of Washington's Juvenile Rehabilitation Administration (2008) stated that reductions in staff caused increased caseloads that affected FFPS fidelity. In the report, Dr. Tom Sexton, cautioned that caseload sizes must range between twelve to fifteen high-risk youth per parole officer, and that quality assurance measures must continue to be in place to maintain program fidelity (Barnoski, 2002, 2004; Rowland, 
2007; Sexton, 2011). Thirty years earlier, Gordon \& Arbuthnot's (1988) study also suggested that large caseloads and workloads diminished the probation officer's ability to adhere to the FFT as required.

This is the first study that examines the implementation of FFPS among juvenile probation officers and examines their views on the advantages and disadvantages of FFPS as a case management model. Since the implementation of FFPS, the Juvenile Court Counselors and Community Justice Managers are required to deliver and monitor FFPS in addition to the range of court and community corrections responsibilities which existed prior to the implementation of FFPS. FFPS requires a broader set of skills and requirements that the Juvenile Probation Officers and Community Justice Managers are required to learn and to deliver, monitor and maintain. It is expected that despite the fact that the organizational context at Juvenile Services Division is conducive to adopt innovative evidenced-based principles, incorporating the Juvenile Court Counselors and Community Justice Managers responsibilities in addition to learning and performing FFPS requires individual and organizational changes that would better foster FFPS implementation.

In addition this qualitative case study serves to describe and explore how closely the Juvenile Court Counselors and the Community Justice Managers were implementing FFPS components and attempts to explain and examine the factors that assist and challenge FFPS program fidelity. In addition, this study recorded the Juvenile Court 
Counselors', Community Justice Managers', and Senior Managers' perspectives, based on their professional expertise, the pros and cons of FFPS as a community-based case management model. The study included observations of consultation meetings and semistructured interviews of 11 Juvenile Court Counselors, four Community Justice Managers, and two Senior Managers.

The primary results of the study suggest that Juvenile Court Counselors and Community Justice Managers perceived observed increases in family functioning and that FFPS is well received by the county's youth and families. Moreover, all staff indicated that FFPS fits the county's philosophy that youth and families should be receiving rehabilitative services while on community supervision. However, Juvenile Court Counselors, Community Justice Managers, and Senior Managers' self-reports indicate that they continue to address remaining individual and organizational challenges (e.g. time management, monitoring, lack of buy-in) during the second year of FFPS implementation.

As a backdrop for understanding the design and implementation of FFPS Multnomah County Juvenile Services Division it is useful to quickly review the national perspective on best practices related to juvenile probation services. In doing so, we need to examine the theoretical reasoning behind implementing family-based interventions, explain Functional Family Therapy, describe the core components and skill sets needed for FFPS, describe the elements which define FFPS program fidelity, and explain 
organizational and individual level factors that assist and challenge program implementation.

\section{Residential Placements and Detention is Costly}

Too often youth are placed in detention facilities or costly residential treatment centers, burdening state and counties with correctional costs and separating families for months at a time (Greenwood, 2008; McGuire, 2010). According to Puzzanchera, Stahl, Finnegan, Tierney, and Sunder (2004) and Puzzanchera and Kang (2010), the number of adjudicated delinquency cases that resulted in residential placement increased $49 \%$ from 1985 to 2000 . As a result of the increasingly high number of youth commitments, $40 \%$ of public and private juvenile residential facilities reported overcrowding in October 2002 with approximately 92,000 delinquents (Synder \& Sickmund, 2006). What is more troubling, Henggeler and Schoenwald (2011) report that $20 \%$ of youth who are committed to residential or therapeutic foster homes were placed because of probation violations, such as not attending school, and missing curfew, but not for more serious crimes. A youth placed in residential or community placement can be separated from their family members up to four months or more prior to returning home (Synder \& Sickmund, 2006). Moreover, critics of residential and corrections commitments argue that aggregating antisocial youth in these locked-down residential facilities can perpetuate criminogenic thinking and iatrogenic affects (Dodge, Dishion, \& Lansford, 2006; Rhule, 2005). Equally important is that residential treatment modalities do not always address 
the youth and family's dynamic risk factors, nor does the residential therapist provide family therapy or understand the environment that the youth will be returning (Henggeler \& Schoewald, 2011). The goals of family-based interventions is to reduce the number of residential placements and detention by keeping youth in the home with their families that gives counselors the opportunity to address the problems in the home that are correlated with juvenile delinquency.

\section{Theoretical Reasoning behind Implementing Family-based Interventions}

A review of the current literature suggests that while there are numerous domains of risk and protective factors that may contribute to youth delinquency (e.g. poor school performance, alcohol and drug abuse, anti-social peers), many of these risk factors for atrisk adolescents are either directly or indirectly family-based or have origins in the home (Crosnoe, Erickson, \& Dornbusch, 2002; Sampson \& Laub, 1993). In fact, many studies suggest that promising treatment approaches for juvenile delinquency are family therapeutic approaches (Gordon, Graves, \& Arbuthnot, 1995). In an in-depth review of the family processes involved in adolescent delinquency and antisocial behavior, Smith and Stern (1997) suggest that lack of parental supervision and monitoring of adolescent behavior is most consistently linked to juvenile delinquent behavior. In addition, the lack of productive communication among family members and the lack of problem solving skills which are associated with delinquency originates within the family (Smith \& Stern, 1997). 
The seminal study of the differences between delinquent and non-delinquent boys conducted by Glueck \& Glueck in the 1950s (cited in Sampson \& Laub, 1993) discovered multiple pathways a child becomes a juvenile delinquent that include a child's personality, temperament, intellect, and family issues to name a few. One of the most important forces found was an unhealthy family environment in the home which include household crowding, large family size, low socioeconomic status, family disruption and parental criminality/drinking/substance abuse. These factors influence the lack of social control of the youth which results in delinquent behavior (Sampson \& Laub, 1993). Sampson \& Laub's own studies on juvenile delinquency suggest that $73 \%$ of the total effects of contextual background factors are mediated by family factors that include ineffective parental discipline, the lack of parental supervision, and the lack of parent/child attachment. In addition, the study found that parental rejection, indifference or hostility toward the child, the use of harsh and erratic punishment, and the lack of follow-through of punishment were shown to be significant risk factors correlated with juvenile delinquency (Kogan, 1980; Kazdin, 2001b).

According to Pollard and Hawkins (1999), interventions should be designed to raise the family's protective factors while decreasing the youth's risk factors. Pollard and Hawkins (1999) analyzed the effects of the risk and protective factors present in the lives of adolescents across five states on substance use and antisocial behaviors, the authors found that increased levels of risk factors were related to higher rates of substance use, 
school problems and delinquency, while higher levels of protective factors were related to lower levels of the same behaviors. Jessor and colleagues (1995) investigated the relationship between psychosocial protective factors utilizing a self-report instrument to measure and compare a youth and family's protective factors versus risk factors. The results showed that higher levels of protective factors relative to risk factors lead to fewer youth behavioral problems. Therefore, programs that address these risk and protective factors aid in reducing recidivism and increasing family functioning (Alexander et al., 1998; Sexton, 2011). To address and decrease family relational factors that are related to juvenile delinquency Alexander and Parsons (1973) developed Functional Family Therapy (FFT).

\section{Functional Family Therapy}

Alexander and Parsons (1973) developed FFT to target populations that range from at-risk adolescents to youth with very serious problems such as conduct disorder, violent acting-out, and substance abuse. FFT is conducted as a phase-driven, behaviorally oriented family program that targets medium to high-risk youth on probation ages 10 to 18 with severe behavior problems and chronic delinquency. The program's success is attributed to its focus on working with the family and youth holistically rather than concentrating solely on the youth. FFT has been applied to a wide range of problem youth and their families in various multi-ethnic, multicultural contexts in the home or in clinical settings as an outpatient therapy (Sexton, 2011). While FFT targets youth aged 
$10-18$ in the criminal justice system, a offender's younger siblings participate in FFT with the intention of keeping younger siblings from becoming delinquent as well.

According to Alexander et al. (1998) FFT's success depends upon specific characteristics of the FFT therapists and resources dedicated to the training and supervision of therapists. Therapists have a caseload of 10 to 12 families and sessions range from eight to 12 one-hour sessions for mild cases and up to 30 sessions for more difficult situations. The characteristics of successful FFT therapists include nonjudgmentalness, persistence, non-blamingness, humor, empathy, multicultural sensitivity, and warmth, as well as a clear understanding of the model and its goals. As for the resources needed for FFT, video or tape equipment must be available for FFT therapists to record family sessions and for managers to watch. These recorded family sessions allow FFT supervisors to provide feedback on the FFT therapist's model adherence. It is recommended that FFT supervisors who monitor FFT therapists should manage no more than five therapists and they must have weekly supervision meetings with their FFT therapists.

FFT's effectiveness has been demonstrated among a wide range of problem youth in numerous trials for over the past 25 years among multiethnic and multicultural youth, using different types of therapists in a variety of social work and counseling professions (Greenwood, 2006; Sexton, Ostrom, Bonomo, \& Alexander, 2000). Sexton, Ostrom, Bonomo, and Alexander (2000) found that FFT -treated youth from diverse communities 
(multi-ethnic and cultural) who had committed drug offenses, violence, and property crimes had between $25 \%$ and $40 \%$ lower levels of recidivism one year later than those who participated in residential treatment. Moreover, FFT cost $\$ 2,325$ per youth and family, providing a criminal justice savings of $\$ 14,600$ (Greenwood, 2008). The latest statistics from Washington State Institute for Public Policy indicated that FFT achieved a total benefit of $\$ 37,739$ per youth. In sum, the benefits of FFT yielded $\$ 8,536$ saved to taxpayers, and gave a total return on investment at $641 \%$ (Aos et al. 2011). Moreover since 1998, after reviewing 900 programs, the Center for the Study and Prevention of Violence published its "Blueprints" research identifying 11 programs that met the rigorous criteria to be named a "best practice." FFT is listed as one of the top three model programs that are cost-effective in reducing recidivism (Center for the Study and Prevention of Violence, 2012).

Functional Family Probation Services Skill Sets and Core Components Using the same skills sets and principals of FFT, Dr. Jason Alexander and Douglas Kopp, CEO (2002) modified FFT into a case-management system called Functional Family Probation/Parole Services (Functional Family Probation Services Manual, 2011). According to the FFPS manual and one of the trainers of FFPS, community corrections professionals receive three-four day training sessions learning FFPS skill sets and principals as well as its quality assurance protocols to ensure program fidelity (Medina, J., personal communication, July 2012). At Multnomah County Juvenile Service 
Division, the Juvenile Court Counselors and Community Justice Managers were trained to deliver FFPS to probationary medium and high-risk youth for up to one year in the youth and family's home. In the county, FFPS services are not mandatory; nor required by court order.

All youth that are placed on probation in Multnomah County receive FFPS services by their assigned Juvenile Court Counselor (JCC). Prior to delivering FFPS, the JCC completes the Juvenile Crime Prevention (JCP) Assessment tool to determine the youth and family's risk level. The JCP is a computer-based initial assessment screening tool that measures the dynamic and static risk factors of youth based on the models developed by Hawkins, Pollard, and Catalano. Questions in the JCP assessment tool include the domains: schools, friends, extracurricular activities, substance abuse, family relationships, and attitudes, values, and beliefs. The JCP Assessment tool is updated every 90 days and youth who receive a medium to high-risk score receive FFPS services. During FFPS sessions community corrections professionals are required to facilitate communication among family members and discuss the factors correlated with juvenile delinquency (e.g. parenting skills, problem-solving skills). The skills and principles of FFPS are listed in the most recent FFPS Training Manual (2011) provided to this investigator during FFPS training includes:

1. Working alliance - The JCC works to maintain a balanced alliance between the youth and parents. The JCC is neutral and does not take sides. 
2. Relational Family focus - The JCC focuses on relationship-building among the youth and his or her family members, community, and peer groups. This requires the JCC to facilitate communication among the family and to teach them how to problem solve and reduce conflict.

3. Strength-based - Instead of focusing on the negative issues within the youth and the families' lives, the JCC emphasizes the protective factors or positive aspects of the family relationship by identifying the strengths of the family. The goal is to reduce blame, attribution, and fighting among the family members. Additionally the objective is to look to the "noble intent" of the family and understand the origin of the conflicts.

4. Respect - The JCC works to respect family dynamics and the ethnic and cultural diversity within families. Youth and families should feel valued and safe in conversations and should be acknowledged for their participation efforts. This is a client-centered approach in that the family brings what they need to the session, as opposed to the JCC bringing what he or she thinks the youth and family needs.

5. Matching - The JCC tailors an approach that matches the family's language, norms, and values that are unique to the family.

Other skills included are the "Change Focus Techniques" that are employed to reduce resistance/blaming and negativity, as well as to decrease hopelessness and 
increase healthy communication (FFPS Probation Model Manual (2011). The examples of "Change Focus Techniques" include:

1. Strength-based relational statements - statements of nobility "noble intents" about one of the family member's efforts toward another person in the family. These are statements that help tie family members together by changing the focus of the conversation away from problems and faults toward the strengths of family bonds. This skill set also increases the balanced alliance with both the youth and family.

2. Interrupt and Divert - do something to interrupt a possible escalation when a family member begins blaming others and is increasing the negativity and hopelessness in the session.

3. Pointing - describe the negativity and meet it in a positive strength-based way and tell and question them what underlies that comment and how that behavior impacts all of the people in the room.

4. Sequencing - describe the negative interaction that occurred outside of the meeting in a strength-based way.

Another technique which is critical for the community corrections professional to master is recognizing the themes that families need to work on and to use FFPS/FFT skills, such as reframing to keep the youth and family focused. Reframe involves the counselor taking the lead in suggesting that a problem behavior may not necessarily have 
a malevolent motive; instead the behavior could be based on a more positive intent. A behavior that is motivated by positive intent is called the "noble intent," which may be used during the reframe technique. Essentially, the problem behavior is a theme and to help families change the meaning of the problem behaviors, the following are the suggested things that counselors can say to families as stated in the FFPS Manual (2011, p. 12):

- Anger implies hurt. Almost every time we see/feel anger, it reflects an underlying hurt.

- Anger implies loss. Anger may reflect a fear of hurt or loss of love, control, sense of trust, sense of family, etc.

○ Defensive behavior implies emotional links. Your mate (child, parent, etc.) acts defensively when he lies because it is difficult for him/her to lie to you. That implies a relationship and sense of caring that s/he can't express directly. That they are defensive about implies that they care and suggests emotional links

- Nagging equals importance. S/he nags because you are so important and because s/he does want you to be close, available, and nondestructive to the relationship, etc. Unfortunately, people tend to drop out/forget the underlying positive reason for the nagging, so that all one hears is the criticism. 
- Pain interferes with listening. When someone is insensitive or selfish, it may reflect the fact they are in too much pain to be able to consider others.

$\circ$ Frightened by differences. Some individuals are afraid of differences between them and other family members because they fear those differences will lead to lack of commitment, loss of control, an unwillingness to continue the relationship, etc.

The skills and principles are delivered in three flexible phases described in the FFPS Probation Model Manual (2011):

1. Engagement and motivation: The JCC delivers FFPS in the first 10 days, 2 to 3 times, the youth is placed on the JCC's caseload. The JCC fills out the FFPS Family Service Plan Part I, II, and III which is a record of the JCCs assessment of the family relationship and the family's criminogenic domains. The service plan has up to eight criminogenic domains the family and JCC must work on during sessions (See Appendices). Afterward, during this stage, the JCC meets with the family one to two times a week depending on their needs.

2. Support and Monitor: The JCC delivers FFPS once a month and continues to facilitate communication among the family and helps them resolve conflict and problem solve on their own. The JCC also monitors the youth and families participation in community services and treatment (e.g., alcohol and drug treatment, counseling, and employment services). 
3. Generalization: The JCC delivers FFPS more frequently in order to keep the family continuing in outside services and to prepare them to maintain positive changes once the youth completes their probation requirements and the FFPS sessions are terminated. 


\section{Functional Family Probation Services Quality Assurance Protocols}

To ensure the Juvenile Court Counselors and Community Justice Managers are implementing FFPS core components effectively and the Juvenile Court Counselors are utilizing FFPS phases and skills sets with program fidelity, FFPS has multiple components to ensure quality assurance: Juvenile Court Counselors and Community Justice Managers attend weekly FFPS consultation sessions; Juvenile Court Counselors complete FFPS paperwork (e.g. service plans, case plans, case notes); with permission Juvenile Court Counselors occasionally record family sessions; Community Justice Managers listen to recorded family sessions; Community Justice Managers observe family sessions of each unit JCC; Juvenile Court Counselors receive ongoing, monitoring and coaching from Community Justice Managers; Community Justice Managers complete the Global Rating Measure for each Juvenile Court Counselor in the Community Justice Manager's unit, and the youth and family complete the Balanced Alliance Questionnaire after the first 30 days of FFPS delivery.

During the FFPS consultation sessions the Juvenile Court Counselors are asked to discuss the family's particular needs and characteristics with the team. Each JCC shares with the team the FFPS techniques that they are using to increase family communication and how these techniques reduce negativity, conflict and hopelessness. During the meetings Community Justice Managers provide coaching and feedback to the Juvenile 
Court Counselors on how to improve their FFPS competence. In addition, each quarter, the unit Community Justice Manager rates each Juvenile Court Counselor's competence by completing the Multnomah County Department of Community Justice Global Rating Measure (see Appendices) created by Alexander, Kopp, \& Sexton (2002) (as cited in Rowland, 2007, p. 80). The Global Rating Measure is a 30-item record of each Juvenile Court Counselor's model adherence and competence with a 4-point Likert scale for each item, which ranges from 0 (low adherence) to 4 (high adherence).

\section{Program Fidelity of Core Components}

Maintaining fidelity of the above FFPS components is critical to keep costs down and to reduce recidivism (Barnoski, 2002, 2004; Sexton, 2011). For example, the results of parole officers model adherence and competence delivering FFPS in the State of Washington revealed that parole officers who demonstrated model adherence and competence reduced recidivism while non-adherent officers raised recidivism (Barnoski, 2004). According to Mihalic et al. (2004) program fidelity during implementation of programs consists of four components:

1. Model Adherence: The program is to be delivered using the skills sets and principles of the model to the correct target population as designed. For example, FFPS is delivered to medium and high-risk families using FFPS skills sets and principles within each phase. Furthermore, Juvenile Court Counselors are required to deliver FFPS in three 
phases and at each phase there is a recommended number of times the Juvenile Court Counselors deliver FFPS to youth and family in their homes.

2. Exposure (referred to as dosage): A model must be delivered to clients with the required amount of exposure. For example, FFPS requires a length of time and a range of a number of family sessions within each phase, depending on the needs of the family. The Juvenile Court Counselors are trained on program content and are provided the recommended amount of time (length for each family session) for each phase of FFPS. For example, FFPS requires that in the first phase, the engagement and motivation phase, Juvenile Court Counselors are to meet for 20 to 45 minutes with the youth and his or her family members two to three times within the first 10 days the youth is assigned to a Juvenile Court Counselor.

3. Quality of Program delivery: Is the manner in how a counselor delivers a program (e.g. the person's skills in using techniques or methods prescribed by the program,their enthusiasm, preparedness, and attitude). The Juvenile Court Counselor attends consultation sessions and shares with each unit member the specific FFPS skills sets they apply to families and what manner they use with families. Case plans require the Juvenile Court Counselors to report the family's specific themes and what skills are applied in each FFPS phase to help adhere high quality and effective deliver. 
4. Participant Responsiveness: Is the extent to which participants are engaged by and involved in the activities and content of the program. The family members are required to make efforts to be available and willing to try the intervention.

For programs to be effective all key components must be implemented with fidelity (Mihalic, et al., 2004). Meta-analysis and evaluations of numerous programs demonstrate that better implemented programs produce better outcomes and that poor delivery of evidence-based community interventions may increase recidivism (Latessa, \& Holsinger, 1998; Latessa, 2004; Lipsey, Howell, Kelly, Chapman, \& Carver, 2010).

\section{Statement of the Problem}

\section{The Importance of Implementation Research}

Evidence reveals that there is a relationship between program integrity and effectiveness (Mihalec, 2002; Petersilia,1990; Rhine et al., 2006). Too often negative findings are often attributed to the failure of the model; however, there is ample evidence that innovations fail due to implementation errors involving such mundane matters as logistical issues, space and equipment, staffing resources, management support, or lack of specific organizational factors required for the interventions successful monitoring and delivery (e.g. culture and climate) (Glisson, 2002; Glisson \& James, 2002; Hemmelgarn, Glisson, \& James, 2006; Mears, Kelly, \& Durden, 2001; Rhine et al., 2006). Widespread implementation of effective programs is unlikely to affect the incidence of violent crime unless there is careful attention given to the quality of implementation (Mihalec, 2002). Unfortu- 
nately, few studies monitor the quality or integrity of program implementation, nor monitor program effectiveness after full implementation (Rhine et al., 2006). Most evaluations are outcome studies, which according to Latessa \& Holsinger (1998), provide much of our knowledge about the effectiveness of programs, but provide little if any useful information about why a program is or is not effective. Moreover, when programs fail the reasons for failure is often ambiguous and even evaluators must question how much of the program was implemented (Petersilia,1990) or whether the program was implemented at all. It may be unnecessary to conduct an outcome evaluation until either the program design or implementation is strengthened (Lowenkamp et al., 2006).

There is a growing body of publications and recently a few case studies that examined program implementation and the organizational and individual-level features (such as leadership, staff, buy-in, climate, structure, and culture) in the criminal justice and human services fields that affect successful implementation and sustainability of programs (Aarons \& Sawitzky, 2006; Farrell, Young, \& Taxman, 2011; Gendreau, Goggin, \& Smith, 1999; 2001; Glisson, 2007; Hemmelgarn et al., 2006; Mihalec, 2002; Petersilia, 1990). Gendreau, Goggin, and Smith (1999; 2001) examined the implementation of several programs in correctional treatment and came up with 32 guiding principles for implementing programs effectively. Some of the authors' conclusions include that an organization has a history of adopting new programs and is innovative, as well as has a decentralized bureaucratic structure. Other recommendations 
include that an agency has few staff turnover, the agency provides the staff the time to run the program efficiently and effectively, and its staff are provided with the necessary resources to perform the program that saves time and reduces effort (Gendreau et al., 1999; 2001). Further all stakeholders must perceive that the adopted program has realistic goals and is addressing a "real" problem and that the program maintains and supports professional autonomy, which is particularly crucial for community corrections professionals.

Case studies examining implementation of family-based interventions applied to delinquents exist mostly in child and mental health services, and these studies frequently point that organizational and individual-level features affect service outcomes (Glisson, 2002; Glisson \& James, 2002; Hemmelgarn et al., 2006). The organizational and individual-level factors that need to be examined during and prior to implementation included IT resources, buy-in, leadership, culture and climate (Zazelli et al., 2008; Glisson \& James, 2002). Nonetheless, statistics on organizations that have attempted to change indicate mostly failure for any change that threatens the public safety and does not guarantee the rehabilitation of offenders is likely to face opposition from stakeholders and resistance (Latessa, 2004) from parole and probation officers.

Human resistance to changes in work habits is common especially among probation and parole officers who tend to gravitate toward strategies supported by their personal experience and who do not easily trust research to inform what they deem works 
(Lynch, 1998; White, 2005). Community corrections professionals are accustomed to working independently and employing their own techniques with little oversight (Szabla, 2007). Although according to Joplin et al. (2004), if there is resistance it may stem from the organization's failure to consider and eliminate barriers that would help community corrections professionals perform their responsibilities.

Additional obstacles to a model's successful implementation and sustainability may originate from community corrections professionals who struggle with the dual role afforded to them or who may suffer from burnout, cynicism and overload (Glisson \& James, 2002). The dual role afforded to community corrections professionals, the both combined law enforcement and social work approaches applied by parole and probation work can be conflicting for some (Clear \& Latessa, 1993; Lynch, 1998). In fact, community corrections professionals who believe their primary objective is to increase public safety, may be resistant to a role that requires they apply techniques readily used by social workers. However, Clear and Latessa (1993) state that there is little evidence that role conflict affects job performance. Research conducted by Clear and Latessa (1993) demonstrates that despite clear role preferences, officers are able to perform both tasks of assistance and control and there was little evidence of role incompatibilities. In addition, child service workers and human service workers who are cynical, burnout, or perceive they are suffering from work overload may not be supportive of a program that feel increases their workload, which in turn affects service outcomes (Glisson \& James, 
2002; Glisson, 2007; Simon, 1993). Fortunately, Glisson (2007) states that there is evidence that an organization can move toward a positive work culture and climate with planned change prior to and during implementation.

Background and Need

\section{Organizational and Individual-level Factors during Implementation}

Implementation of a new model and the organizational changes required to ensure program fidelity can take from two to four years (Fixsen et al., 2005). Implementation is difficult and organizational change that fosters successful implementation is even harder especially for corrections agencies (Latessa, 2004; Lerch, Viglione, Eley, James-Andrews \& Taxman, 2011). It is difficult for criminal justice organizations to change because criminal justice agencies have many stakeholders, special interest groups (e.g. victim advocacy groups), and state legislators (Lynch 1998, Mintzberg, 1993) that have varying opinions on what is most effective in keeping the public safe. Any change that stakeholders feel that will jeopardize public safety and does not guarantee timely rehabilitation or incapacitation of criminal offenders is likely to face opposition (Latessa, 2004; Marion \& Oliver, 2006 ; White, 2005).

Although small changes in practices can be made with little concern for the culture of the organization, any changes that do not conform to existing culture and climate may likely meet with unnecessary difficulties or failure (Farrell et al., 2011; Hemmelgarn et al., 2006; Schein, 2004). Organizational culture refers to the shared 
norms, beliefs, and behavioral expectations that drive behavior and communicate what is valued in an organization (Cooke \& Szumal, 2000; Glisson, 2002; 2007; Hemmelgarn et al., 2006). Organizational climate exists when psychological climate perceptions are shared among workers within a particular work unit (Glisson, 2007). Human services research on climate has created quantitative measures that measure an organization's negative climate, which includes role conflict, work overload, burnout, resistance, and exhaustion (Glisson, 2007; Glisson \& James, 2002). Thus, a negative climate with a highly burnt out workers and a culture not conducive towards evidence-based rehabilitative practices may be doomed for failure.

In fact, Lynch (1998) states that "research indicates that organizational policy set by management ... does not get implemented without some reshaping by workers who are responsible for carrying out policy-related tasks" (p. 843). Essentially, the frontline workers, the parole and probation officers who do not deem the policy or program effectual will subvert or downplay the program or policy that they consider problematic or unimportant. This is why the culture of parole and probation officers and the climate of the organization is critical to address while implementing new policies and programs. Therefore, it is useful for organization leadership to address the individual-level and organizational-level factors such as culture and climate that may influence the failure or the success of program implementation. 
Clear and Latessa (1993) found that the organizational philosophy plays an important role in determining officers' attitudes and task preferences. Specifically, an organizational philosophy of treatment is more instrumental in producing support tasks than an officer's personal preferences and most importantly the organizational culture that supports rehabilitation may be the only way to ensure the probation officer adheres to the organization's mission. Nonetheless, organizational culture scholars emphasize that staff resistance in all private, public and non-profit industries is common and is the most significant factor that impedes new programs success or causes excessive adaptation that degrades fidelity (Elliott \& Mihalic, 2004; Szabla, 2007). Officers may be resistant to new ways to work with youth if they do not have faith that a program will be effective or increase public safety (White, 2005). Thus, steps must be taken and the practitioner must be convinced that the benefits of a new program will outweigh the costs (Petersilia, 1990).

Fixsen et al. (2005) states that frequent training and coaching are the principal ways to reduce resistance and to assist in changing work routines and changing work behaviors. Carefully selected staff in the beginning stages of implementation and throughout the life of evidence-based programs can assist in coaching and training. Still, to accomplish this employees must have faith in the organization's ability to reform and provide the resources for staff. Employees must have faith in its leadership, and they must receive frequent training and coaching. Studies conducted by Petersilia (1990, p. 
130) have provided implementation guidelines to reduce resistance at the organization and individual-levels to ensure program fidelity:

1. Sincere motivation at initiation. Adoption of programs must be related to the goals and mission of the agency. Agency administrators that have used innovation as a mean to obtain funds that are then not used for the programs purposes to gain local political support, will not receive support from stakeholders who are delivering the model.

2. Support from top leadership is crucial and resources from all levels of the organization must be devoted to the new mode. An organization must have a leader who is committed to the objectives of the program who can motivate and effect change, for good leaders must pull together those who believe in the program to reduce resistance.

3. Staff must be willing and competent. Resources must be dedicated to constant training and education and also the capacity and willingness to participate in the trainings.

4. A benefit/cost surplus - for all the stakeholders, the benefits of implementing the innovation must outweigh its costs relative to the option to returning to the status quo. There must be incentives given for the new model, particularly if the new model increases workload. This will reduce resistance and staff will grow accustomed to the intervention. 
5. Continual clarity of goals and procedures. Continual articulation of goals and procedures are necessary to ensure fidelity. Additionally, it is important that goals are not set too high in order to gain full support. If goals are set too high and are not met, resistance and loss of faith will ensue. Moreover, the goals must take into account the needs of the client (youth and family) and how it will help them.

6. Clear lines of authority. Champions and leaders for the new intervention must be given powers at resolving differences and problem solving.

7. There must be a close fit between a program and the culture in which it is to be enacted. The closer the fit, the better a programs chances of successful implementation. This notion of fit applies to the structure of the program, the alignment of it is authority structure with the larger system, commitment from all staff and faith in the program, believe in the theory that drives the program and that it will work. Moreover, small changes including small changes in organizational structure and culture are more likely to gain acceptance rather than broad changes.

8. There is low turnover among staff, the technology, and practitioners are trained and competent. Insofar as the deliverer makes the program their own rather than being coerced into, they are not resistance and are bought into the program by being given incentives to maintain program fidelity. 
9. The program addresses an existing "real" problem. The motivation for change and commitment must be strong enough to balance the pressures that change imposes. Projects should be implemented in small, simple steps and the organization should not try to make several changes simultaneously. If staff is pulled in too many competing directions and are unable to devote the necessary time and energy into making any one change really work, it gives the message of mixed goals, thus decreasing the likelihood of the program producing successful outcomes.

In addition to the above implementation recommendations from Petersilia (1990, p. 130), a series of authors have conducted studies and performed meta-analyses that revealed that implementation is key to successful outcomes. Studies conducted by Byrne, Lurigio, and Baird (1989) on the Intensive Supervision probation and parole programs in Georgia, New Jersey, and Massachusetts underscore the value of assessing the extent of program implementation in order to ensure program fidelity. Another evaluation by Byrne and Kelly (1989) created an implementation scale and used it to examine variations in programs for the Intensive Supervision Program operated in 13 sites throughout the state of Massachusetts. They found an inverse relationship between program implementation and offender recidivism. In other words, the more fully the program was implemented the more likely recidivism decreased significantly. Durlak and Dupre (2008) conducted a meta-analysis to assess the impact of implementation and factors that affected program 
outcomes. Their results from over 500 quantitative studies offered strong empirical support that the level of implementation affects program outcomes. In addition Durlak and Dupre's (2008) findings revealed that there are 23 contextual variables that influence implementation, of which the main influence being individual and organizational functioning.

Other notable findings by Lipsey (1998) and Lipsey and Wilson (1998) indicated that expecting near-perfect implementation is unrealistic. In his meta-analyses on juvenile programs, Lipsey and colleagues (2010) found that the most outstanding programs reduced recidivism rates by $40 \%$. In fact, Lipsey noted in his 2009 publication that a well implemented program of an inherently less efficacious type can outperform a more efficacious program that is poorly implemented. Moreover, recidivism reduction was associated with the duration of the service and the total contact hours of service and programs with smaller effects had high drop out rates, staff turnover, poorly trained personnel, and incomplete service delivery (Lipsey et al., 2010). Therefore, Lipsey et al. (2010) concluded that practitioners and researchers would do well to pay attention to how programs are implemented, for quality of implementation are key to larger effect sizes.

To date, only two studies have examined community corrections officers delivering family-based interventions: model adherence and competence of juvenile parole officers in the State of Washington delivering FFPS (Rowland, 2007) and Juvenile Probation Officers delivering FFT in Atlanta Georgia (Gordon \& Arbuthnot, 1988). The 
State of Washington rigorously examined FFPS parole officers' program fidelity since Functional Family Probation Service's implementation in 2002. The results showed that the combination of parole officers' adherence and a measurement of the youth's risk to re-offend explained a significant portion of the variance in post-parole criminal severity (Rowland, 2007). However, the other studies conducted for the State of Washington indicated that poor delivery of FFPS and FFT did not significantly reduce recidivism (Barnoski, 2002b; 2004) In fact, poor delivery and incompetence raised recidivism (Barnoski, 2004). To improve model adherence, reports from Washington indicated that structural changes were necessary for FFPS program fidelity, such as instituting more effective monitoring by hiring trained and experienced FFT staff to more effectively monitor and rate counselors. However, there was no examination of parole officer's experiences in delivering FFPS or an examination of the organizational and individuallevel factors that assisted or created barriers to program fidelity. Nor do these studies include a record of the parole officers' experiences and opinions of FFPS as a case management model.

The second study conducted among Juvenile Probation Officers in Atlanta, Georgia delivering FFT services examined the initial training and implementation (Gordon \& Arbuthnot, 1988). Gordon \& Arbuthnot (1988) found that the Atlanta probation officers could be trained to deliver FFT services and those probation officers with positive attitudes and who were less cynical were better than probation officers who 
were cynical and who lacked empathy towards the youth and family. Other findings indicated that the probation officers struggled adhering to the model because they did not receive support from the bench and their chief probation officers. Moreover, their workload prevented them from delivering FFT with the required dosage and frequency. Overall, the lack of support from the bench and superiors and the increased workload of FFT was associated with the eventual elimination of the program.

\section{Purpose of the Study}

This was a case study conducted during the second year of FFPS's implementation at Multnomah County Juvenile Services Division, in Portland, Oregon. The goals of this study were threefold:

1. First, this project recorded the Juvenile Court Counselors' and the Community Justice Managers' perspectives and experiences on the use and implementation of Functional Family Probation Services.

2. Second, this study examined how closely the Community Justice Managers and the Juvenile Court Counselors self-reported implementing FFPS program components to better inform implementation.

3. Third, this study recorded the Juvenile Court Counselors', Community Justice Managers', and Senior Managers' opinions on what additional individual and organizational changes were critical to improve FFPS core components. 
Observations of FFPS consultation sessions were included to corroborate the Juvenile Court Counselors' and Community Justice Managers' opinions, understandings, and experiences.

\section{Research Questions}

1. What are the Juvenile Court Counselors', Community Justice Managers', and Senior Managers' opinions and experiences with the implementation of FFPS?

2. What are the Juvenile Court Counselors', the Community Justice Managers', and the Senior Managers' experiences on what individual and organizational factors assist or preclude the implementation of FFPS?

3. What are the Juvenile Court Counselors', Community Justice Managers', and Senior Managers' opinions on the advantages and disadvantages of FFPS as a case management model?

\section{Ethical Considerations}

Portland State University’s Human Subjects Review Board approved this study. The ethical issue that could have arisen is that this researcher has collected data and performed evaluations on other programs for JSD senior managers and for the Department of Community Justice. Therefore, some participants could have feared that their opinions may not be confidential.

To allay the fears of participants, this researcher obtained informed consent and stated that the participant's opinions, experiences, and statements were confidential and 
that their participation in the study will remain anonymous. In addition, it is common for quotes to be used in qualitative results to reflect the richness of the results. This investigator did not use quotes that would be recognizable to participants. It was stated to JSD managers and participants that the confidentiality and anonymity of all interviewed staff is of prime importance and that confidentiality is ethically required in any research project. 


\title{
CHAPTER TWO
}

\author{
Literature Review
}

\section{Introduction}

Bringing family-based models into the community requires individual and organizational changes expedient for a models successful implementation and sustainability (Zazzali et al., 2008). Frequent process evaluations during implementation can determine what organizational phenomena assist or preclude program fidelity in the delivery, monitoring and maintenance of program components (Mihalec et al., 2004; Fixsen et al., 2005). Individual and organizational phenomena and factors such as staff resistance, lack of technology, and a culture and climate not conducive for evidence-based practices frequently can preclude program fidelity (Hemmelgarn et al., 2006; Joplin, 2004). On the other hand, innovative organizations with supportive leadership, resources, and a dedicated trained staff model are one of the many factors that can assist successful implementation and sustainability of a program (Fixsen et al., 2005; Joplin et al., 2004). Until recently little emphasis has been given to implementing programs and because of this there is a lack of understanding which components of programs are responsible for reductions in recidivism (Mihalec, 2002). However, what we do know is that research on family-based interventions such as MST and FFT suggest that frequent coaching and consultations sessions as well as the frequency of sessions and dosage moderate family-based 
interventions cost-effectiveness in reducing recidivism (Barnoski, 2002; 2004, Henggeler et al., 1997; Henggeler et al., 1999; Rowland, 2007; Sexton, 2011). In fact, Barnoski’s work (2004) suggests that counselors and community corrections professionals that do not adhere to the model can actually raise recidivism. Studies further indicated that for the model's success staff must be willing to perform the program model and the model must be perceived by the staff that the program will be beneficial (Petersilia, 1990). Moreover, evidence shows that counselors that believe in the intervention and who demonstrate warmth and empathy toward their youth and family clients are successful in reducing recidivism (Alexander et al., 1998; Gordon \& Arbuthnot, 1988). Other studies conducted by Glisson (2002; 2007) and Hemmelgarn et al. (2006) suggest that a positive culture and climate are expedient for the successful implementation of evidenced-based practices.

To date, two studies examined juvenile community corrections professionals delivering family-based interventions: the model adherence and competence of juvenile parole officers in all 14 counties in the State of Washington (Rowland, 2007) delivering FFPS and another study examining juvenile probation officers delivering FFT in Atlanta, Georgia (Gordon \& Arbuthnot, 1988). There are no studies that examined the organizational and individual-level factors among juvenile probation/parole officers that assist or preclude FFPS implementation and program fidelity. Although there are studies that examined the individual and organizational factors during implementation of FFT 
among functional family therapists and social workers at human services organizations (Duncan, Davey, \& Davey, 2011; Zazalli et al., 2008). The evidence from these studies identified a multitude of factors including the individual-level and the organizational contexts that facilitate and preclude program fidelity of FFT that were similarly identified and described in this present study examining Multnomah County Juvenile Court Counselors, Community Justice Managers, and Senior Managers.

This literature review examines six studies that address three areas of research that surround therapists and community corrections professionals delivering family community-based interventions toward juvenile delinquents and their families. The first section presents two studies on juvenile probation officers delivering FFT in Atlanta, Georgia (Gordon \& Arbuthnot, 1988) and juvenile parole officers delivering FFPS in the State of Washington (Rowland, 2007). The second section examines two studies that address the individual and organizational factors that assist the adoption and implementation of FFT as well as the advantages and disadvantages of FFT as an intervention among counselors in human service organizations (Duncan, Davey, \& Davey, 2011; Zazelli et al., 2008). These studies were chosen for this literature review because FFPS is based on the principles and skills sets of FFT. The third section presents two studies on how individual and organizational phenomena affect the adoption and implementation of evidence-based programs in criminal justice and human service organizations that serve juveniles and their families (Aarons \& Sawitzky, 2006; Farrell, 
Young, \& Taxman, 2011).

Juvenile Probation/Parole Officers delivering Family-based Interventions

Gordon and Arbuthnot (1988) conducted FFT trainings of juvenile probation officers in Atlanta, Georgia. The probation officers that voluntarily chose to be trained in FFT participated in two-day workshops and attended weekly supervision meetings and booster workshops. The trainees were predominantly black women, married with children, and who had no formal mental health training. The training required juvenile probation officers to complete a handout for each session that acted as both a summary of their activities during family sessions and as a guide on the development of their FFT counseling skills. In addition, FFT required trainees to mail audiotapes of each family session along with self-report forms to their supervisors. Together with the audiotapes and the summary forms that were sent to a supervisor, the supervisor would give feedback and consult with the FFT juvenile probation officer. The feedback is intended to shape the trainee's behavior primarily by reinforcement and the supervisor role-playing appropriate ways of dealing with what occurred during the session.

Gordon and Arbuthnot (1988) indicated that most of the juvenile probation officers who voluntarily participated to be trained in FFT in Atlanta, Georgia "fared well." During the training, the authors noted areas that assisted the juvenile probation officers' learning and delivery of FFT to youth and families. The authors found that probation officers that had specific characteristics and qualities were effective in delivering FFT. 
These specific characteristics included buy-in and enthusiasm for the techniques and skill sets required of FFT and included those who exuded warmth and empathy towards the youth and family. Those who were curious about FFT and demonstrated the desire to learn during the training sessions demonstrated their grasp of FFT skills. Other noted areas that increased the juvenile probation officers' FFT skills included the high quality and frequency of management supervision and monitoring.

However, probation officers that were cynical and did not believe in FFT did not "fare well." Often cynicism was seen among juvenile probation officers who had held their positions for a long time among low-income serious delinquents. Cynicism and lack of belief in FFT as an effective intervention for the low-income serious delinquent population was demonstrated when trainees frequently provided excuses about why they had to delay starting with a family. It appeared that the longer a trainee worked with serious juvenile delinquents, the more likely the probation officer would not be effective in delivering FFT.

Gordon and Arbuthnot (1988) stated that despite the juvenile probation officers who performed "fairly well" in delivering FFT, there were individual and organizational-level barriers that precluded delivery. The authors noted that the juvenile probation officers did not receive support from the bench or from their supervisors. It was observed that the juvenile judges and the Chief Probation Officer did not provide regular release time for probation officers to conduct family sessions. Then, when caseloads increased 
the juvenile probation officers' motivation to donate the time to the families diminished. Moreover, other court duties and responsibilities kept juvenile probation officers from seeing the youth and families on a regular basis.

To allay some of the above problems among the juvenile probation officers in Atlanta, Gordon and Arbuthnot (1988) recommended some changes to improve FFT delivery. The first suggestion to prevent pessimism was that the weekly consultation sessions were designated as mandatory. The authors noted that the weekly consultation sessions served to improve the juvenile probation officers' quality of delivery, but also the meetings provided a place for the juvenile probation officers to give each other emotional support and feedback on coping with tough family cases. Other suggestions included that there be continual evaluations that can serve to troubleshoot staff needs. For instance, the reliance on home-based treatment delivery can change when juvenile probation officers feel vulnerable entering particular neighborhoods at night. Some of the family sessions can be scheduled at a safer location (school, church, courthouse) until day sessions can be arranged. Overall, Gordon and Arbuthnot's (1988) experience in Atlanta showed that juvenile probation officers can be trained to deliver FFT. However, after the training and because of workload conflict and the lack of support from management and the bench, the FFT program was eliminated (Gordon \& Arbuthnot, 1988).

Gordon \& Arbuthnot's (1988) article was a narrative on their experiences in training juvenile probation officers delivering FFT. Their discussion included the individual 
and organizational factors that precluded and assisted program fidelity of FFT. However, this study did not include how many juvenile probation officers were trained and their opinions on the advantages and disadvantages of FFT.

In 2002, FFPS was implemented among juvenile parole officers in all 14 counties in the State of Washington (Rowland, 2007). Rowland's (2007) study examined the efficacy of FFPS as a case management model among Washington's juvenile parole officers as compared to traditional parole services. This was the first study to investigate the effectiveness of a treatment program at the juvenile parole level, for few studies examined juvenile community corrections interventions at the community after-care level. Rowland's (2007) literature review showed that previous research suggests that the competency of the counselor or therapist is a moderator of behavioral outcomes (Rowland, 2007). Moreover, treatments developed in the field of psychology suggest that interventionists must become skilled at executing the model as prescribed in the manual (Corrigan, Steiner, McCracken, Blaser \& Barr, 2001 as cited in Rowland, 2007). Rowland's (2007) study was an attempt to determine that counselor competency moderates outcomes and that strict adherence to a model's manual is imperative for reducing recidivism. Rowland's (2007) dissertation listed two of its study's goals: (1) examine the model adherence and competence of the juvenile parole officers, and (2) examine the role of parole officer model adherence as a predictor of post-parole juvenile behavior and family functioning to determine FFPS's efficacy. 
The data for Rowland's study was provided by the Washington State Juvenile Rehabilitation Administration (JRA) that provided information on 60 (originally 81) juvenile parole officers and provided crime information (crime severity index, parole revocations, recidivism) on 621youth. The parole officers in the study had completed the FFPS two-day training provided by FFT Inc. Subsequent to the training the juvenile parole officers attended weekly consultation sessions and completed FFPS case notes. The case notes are monitored by the counselors' supervisor. Typically the juvenile parole officer is required to meet with the youth family three to four times in the first six weeks the youth is paroled to his or her home. Afterward, the juvenile parole officer is required to meet with the family once per month until the last month of parole. In the last month, they are required to see the family twice. For high risk youth, the state law requires that the juvenile parole officer make contact with the youth at least once per week throughout all phases of the FFPS model.

The data on the FFPS paroled youth were collected on 621 youth for at least 30 days. Another 621 non-FFPs youth on parole were matched to the FFPS youth as the comparison/control group. All youth and the matched comparison/control group according to the Initial Security Classification Assessment Score (ISCA), were all high-risk. Rowland (2007) explains that the comparison group was a historical one, comprised of youth who previously received either intensive parole service, regular parole services, basic training camp, or were on sex offender parole conditions. These youth were randomly 
selected based on a stratified sampling procedure. The historical sampling procedure was necessary because FFPS is provided to all youth once they are on parole. Researchers at Washington State Institute of Public Policy (WSIPP) matched youth using a computerized algorithm in order to obtain matched historical youth which were then forwarded to Rowland. The 621 historical non-FFPS comparison/control youth were matched on age, gender, race/ethnicity. In addition, WSIPP provided information for both the FFPS group and the non-FFPS comparison/control group that encompassed the youths' prior history of offenses and the youths' offenses during parole. The information regarding the offenses included crime history, date and the type of crime, and the charges before and after parole services. The primary independent variable was the treatment received (FFPS or nonFFPS youth) and the dependent variables consisted of measures of recidivism, crime severity, and parole revocations. Although recidivism (repeat criminal behavior) often results in revocation, recidivism and revocation variables were analyzed separately as recidivism data was collected after the completion of parole, while revocation data was collected during a youth's time on parole. Parole officer model adherence and competence were recorded in the Functional Family Parole Global Rating Measure (GRM) by FFPS program managers, FFPS region leads, and from the FFPS state-wide consultants to identify the role of treatment adherence in outcomes.

The GRM created by Alexander, Kopp, \& Sexton (2002) (as cited in Rowland, 2007, p. 80) "is an observational instrument for quantifying parole counselor behavior 
with regard to their level of adherence to the fundamental clinical components of the FFPS model.” The FFPS GRM is a 34 item assessment with a 4-point Likert scale for each item, which ranges from 0 (low adherence) to 4 (high adherence). The measure is divided into five sections. The first four sections asks the observer to rate the parole counselor adherence to the fundamental goals and skills of each of three phases of the FFPS model. The final section of the instrument requires the observer to give an overall rating of the parole counselor's adherence to the model. An administrative assistant uninvolved in the present study was responsible for removing identifying information from the assessment measures and assigned each parole counselor a unique identifier in order to maintain confidentiality.

Several statistical tests were employed to determine statistical significance of the outcome measures and to determine if juvenile parole officer model adherence and competence was a moderator of behavioral and family functioning outcomes. To determine if parole counselor adherence to the FFPS model moderates the outcomes of youth and families, group means were generated of parole counselor model adherence for three times periods (first year of adoption, second year of adoption, and third year of adoption) using ANOVA for analysis. Differences between the parole counselor adherence to the model (dependent variable) were compared between the three time periods, which was the independent variable. To determine if FFPS is a more effective intervention compared to traditional parole services, Chi-square analysis was employed to compare the observed 
frequencies of youth who recidivated in each category (misdemeanor or felony recidivism) between the two types of treatment (FFP and non-FFPS youth). To determine if FFPS would reduce crime severity from pre- to post-treatment when compared to those youth in the comparison parole group, repeated-measure analysis of variance (ANOVA) was employed. To determine the number of parole revocations multivariate analysis of variance (MANOVA) was employed. The use of MANOVA was needed because the number of revocation days were believed to be related to the number of times a parolee had his or her parole revoked. Further, to adequately answer this research question, the type of parole standards (e.g. sex-offender parole) also had to be considered, as different levels of supervision and required parole officer contacts may have impacted opportunities for parole requirements to be violated. To determine post-parole criminal severity score and recidivism outcomes, simple linear and logistical regression were employed. Linear regression was conducted using parole counselor adherence to determine the predictive value of parole counselor adherence to the FFPS model in explaining subsequent behavior. The second analysis used logistical regressions to address the role of treatment adherence. Logistical regression was used to identify potential differences in misdemeanor and felony recidivism rates (dichotomous variables) of youth receiving FFPS from one of four groups of FFPS parole counselors (not adherent, borderline adherent, adherent, and highly adherent) as compared to the comparison/control non-FFPS group.

To determine adolescent and family functioning across all domains, the study util- 
ized the Client Outcomes Measure (COM) and the Parole Outcomes Measure (POM) forms. The COM and POM are completed by the youth and family to measure general family functioning. The COM are self-reports of the youth and families perceived family functioning. The POM measures general perceived family functioning from the perspective of the parole counselor. The limitation in the design is that to compare family functioning changes between those youth who receive FFP S and those youth who receive traditional parole services (non-FFPS youth) was not possible. Measures of central tendency (mean and standard deviation) were employed. The within treatment group family functioning outcomes as determined by client and parole officer's self-reports are described by average reported changes in the domain of family functioning.

The results of Rowland's (2007) study indicated that highly adherent juvenile probation officers increased family functioning and reduced a youth's post parole criminal severity index. However, Chi-square analysis suggested that FFPS and the matched compared groups (non-FFPS youth) did not differ in overall recidivism. To determine if FFPS is an effective intervention as compared to traditional parole services, as measured by the juvenile crime severity index (crime type and frequency of crime), repeated measure ANOVA was conducted, with the two levels of crime severity index scores (pre- and posttreatments) as the within participants variable, and treatment group as the between participants variable. Results indicated a significant main effect for crime severity, showing that the severity of criminal behaviors for youth for both treatment groups involved in the 
study decreased from pre- to post-treatment $(p<.001)$. However, no significant differences were found between treatment groups in the changes in severity of criminal behavior before and after treatment. To determine differences in the number of revocations and the number of days parole was revoked between the treatment groups, a 2 (treatment and control) x 2 (types of parole standards: basic training, intensive parole, regular parole, and sex-offender parole) MANOVA was conducted. The MANOVA results suggest a significant main effect of treatment group on the number of revocations a youth received. Results also suggest a significant main effect of parole type on the number of revocations a youth receives. However, no significant interaction was found between treatment group and parole type for either the number of revocations a youth receives $(p<.05)$ or the number of days parole was revoked. Therefore, results suggest that both the type of treatment an individual parolee received and the type of parole an individual was on both had independent effects on the number of parole revocations. Overall, those who received FFPS had significantly fewer parole revocations than those youth receiving traditional parole services.

To determine family functioning outcomes (a within group measure), descriptive statistics from each perspective (adolescent, parent, and parole counselor) on each of the items addressing the major risk factors for adolescent delinquent behavior were used.

Overall, parents receiving FFPS reported in the COM that they had observed changes in their family that they described as between "somewhat better" and "a lot better" prior to 
the FFPS intervention $(\mathrm{N}=133, \mathrm{M}=3.44, \mathrm{SD}=1.19)$. Adolescents receiving FFPS reported in the COM that they observed changes in their family and the youth described as "some better" $(\mathrm{N}=170, \mathrm{M}=3.27, \mathrm{SD}=1.18)$. Using the scale provided to the adolescents, this rating demonstrates that adolescents experience success in some of the things they tried to change within their family during parole. To determine if parole officer model adherence was a moderator for behavioral outcomes across the three times, a one-way ANOVA was used. The adherence scores were divided into three groups: first year of FFPS adoption, second year of FFPS adoption, and third year of FFPS adoption. The results indicated a significant main effect over time since the first year of FFPS services $(\mathrm{F}(2,1091)=12.61, p<.001)$. At each subsequent time period the study suggests that there was an increase in parole counselor adherence to the FFPS model after the first year of its adoption.

To determine if parole counselor model adherence explains the variance in posttreatment criminal behavior of those youth who received FFPS, two separate analysis were conducted. The first analysis employed linear regression which sought to understand the predictive value of parole counselor model adherence and competence to the FFPS model in explaining post-parole criminal severity. The second analysis employed logistical regression, which attempts to illustrate the differences in adjusted felony recidivism rates of youth receiving FFPS from one of four groups of FFPS parole counselors (not competent, borderline competent, competent and highly competent) compared 
to the matched comparison group. The results showed that the combination of parole officers' adherence to the model and a measure of the youth's risk for re-offense explained a significant portion of the variance in post-parole criminal severity among the treatment group (Rowland, 2007). The results of logistic regression analysis, although not statistically significant, demonstrate a $14.6 \%$ reduction in 12 -month felony recidivism rates for those youth who received FFPS from a highly-adherent parole counselor in comparison with those youth receiving traditional parole services (JRA, 2008; JRA, 2012; Rowland, 2007).

Rowland's (2007) overall results revealed family functioning increases and those youth who received FFPS services from a highly adherent juvenile probation officer had significantly fewer parole revocations than youth receiving traditional parole services, suggesting FFPS's potential for reducing costs. This study also showed that parole officers can be trained to deliver the model and that as the parole counselor's adherence to FFPS increased, there was a correspondent and significant decrease in the post-parole criminal severity of the youth (Rowland, 2007). Rowland's (2007) findings suggest that model adherence and competence moderates a youth's post-parole criminal severity. Furthermore, comprehensive quality assurance protocols are needed to monitor ongoing adherence to increase the likelihood of achieving the expected results of FFPS.

Rowland's (2007) study, although extensive and ground-breaking, has several limitations. For many parole counselors the GRM form was not completed for Rowland to 
receive an overall GRM rating for each parole counselor in the study. Most of the ratings on the parole counselor were in the Engagement and Motivation Stage. There was few ratings on counselors in the Support and Monitor and Generalization stages. Moreover, Rowland's (2007) analysis of the inter-rater reliability of the raters were significantly different in the Engagement and Motivation Stages. Further, many parole officers were never rated at all and had to be dropped from the analysis. Finally, youth were referred to other services during later stages of the model and Rowland did not have that information, for the author could not assess the impact of the different services the families received.

The literature has pointed that evidenced-based programs should fit the organizational contexts and philosophy of a criminal justice organization (Fulton et al., 1997; Lowenkamp \& Latessa, 2005). The study by Rowland (2007) examined model adherence and competence, but did not examine the organizational phenomena that may assist and preclude of Functional Family Probation Services components at the organizational and individual-level among probation officers. Implementation studies indicate that organizational factors such as culture, climate, structure, as well as reduced resistance and buy-in assist in successful implementation of program components (Glisson, 2002; Joplin et al., 2005).

The Adoption and Implementation of Functional Family Therapy 
The following two studies examine the importance of program implementation and the individual and organizational-level contexts that affect implementation of community-based family models in human service organizations (Duncan et al., 2011; Zazzali et al., 2008). The two studies were chosen for this literature review because many of the individual and organizational-level barriers and facilitators in implementing FFT in human service organizations were similar to the barriers and facilitators experienced by the juvenile court counselors and the community justice managers during the the second year of FFPS's implementation at Multnomah County Juvenile Services Division in Portland, Oregon.

Duncan et al. (2011) state that evidence-based practices are appealing to agencies; however, a major limitation in implementing evidence-based programs is the ability to translate these models designed in the lab and university into community-based settings. To have a better understanding of the organizational contexts that affect program fidelity of a family-based intervention, Duncan et al. (2011) examined the advantages and disadvantages of FFT, as well as the facilitators and barriers in implementing FFT at five community mental health agencies located in the northeastern part of the United States. The study consisted of two research questions: (1) What are the experiences of transporting an evidence-based program into community-based settings for administrators, supervisors, and clinicians? (2) What are the facilitators and barriers to implementing FFT in community-based settings for administrators, supervisors, and 
clinicians?

The Duncan et al. (2010) study was conducted in two steps. The 18 participants consisted of three levels of staff (administrators, supervisors, and clinicians) who participated in a web-based survey using a modified Delphi method. The Delphi method is a widely used mixed method of gathering group consensus from a panel of experts (Tersine \& Riggs, 1976). The original Delphi method entails three phases of data collection, but to reduce fatigue and participant attrition, the authors modified the method to include two survey rounds. The first phase was an open-ended phase in where participants generate innovative responses to questions. The phase one questions consisted of demographic information, a brief description of the participant's agency, and the following six open ended questions: (1) From your experience, either within this program or through other similar programs, have you experienced any advantages of implementing FFT into your agency? (2) From your experience, either within this program or through other similar programs, have you experienced any disadvantages of implementing FFT into your agency? (3) From your experience, either within this program or through other similar programs, in your opinion, are there ways in which FFT is (was) an appropriate fit for your agency? (4) From your experience, either within this program or through other similar programs, in your opinion, are there ways in which FFT is (was) not an appropriate fit for your agency? (5) From your experience, either within this program or through other similar programs, in your opinion, are there ways in which 
the implementation of FFT has been helpful to your clientele? (6) From your experience, either within this program or through other similar programs, in your opinion are there ways in which the implementation of FFT has not been helpful to your clientele? If so, in what ways? Then the researchers categorized the open-ended responses. Phase Two was a second follow-up survey and asked the participants to rate items using a Likert-type scale to identify areas of consensus.

Duncan et al.'s (2011) results revealed the advantages and disadvantages of FFT and what facilitator and barriers occurred during FFT implementation. Some of the overarching disadvantages for therapists: (1) FFT has too many guidelines and involves too much paperwork. There is too much paperwork that requires therapists to fill out forms after each session to ensure model adherence and to constantly reflect on the goals of each session; (2) many families need to be at a higher level of functioning so they are able to understand and commit to behavior change. In other words, if a family's protective factors are too low and there is too much conflict or crises in the family, the family may not be able to work at changing their behavior or be ready for any change at all; (3) many families have severe drug and alcohol, and mental health problems, which hinders the delivery and effectiveness of FFT. These problems hinder effective communication and the level of help they need is much higher than FFT can provide; (4) therapists cannot get the whole family together required by the model because of work schedules or because of family instability or lack of any togetherness; (5) FFT is not 
helpful for families who don't want the service (involuntary clients). Families will purposely cancel appointments or not be at home when therapists arrive, and finally, (6) therapists report that because of the complexity of the model, counselors ask for more frequent training, consultations, and practice in delivery FFT. The advantages of implementing FFT: (1) families communicate better and fight less and are able to learn how to problem solve and reduce conflict, (2) funding sources want evidence-based practices that are cost-effective, (3) FFT helps families with a broad range of backgrounds and a range of problems, (4) FFT gives clients who are involved in the legal system services that they would not otherwise receive, and finally (5) FFT requires a client-centered and respectful approach.

In sum, participants for phase two of the study reached a consensus that they were able to remedy conflicts and issues in implementing FFT because these organizations already had the structure and philosophy in place for FFT, but they all still struggled with FFT's additional paperwork and workload. The consensus on the ways in which FFT was an appropriate fit for their agencies: (1) being able to help families increase their communication is beneficial not only for the youth but also for the entire family, (2) the family-focused model fit all the agencies' philosophies, (3) FFT is suitable for the agencies because they already work with children who are experiencing a variety of problems because of being removed from their home, being on probation, or from having psychiatric problems, (4) like FFT, the agencies are focused on community-oriented 
services, and (6) the strength-based approach fits the agencies' philosophies.

A noteworthy limitation to the Duncan and colleague's (2011) study was the small and unbalanced sample size across all levels of FFT staff. Additionally, the participants did not have enough expertise based on the average length of time implementing FFT (1 year), although the individual characteristics of the family therapists were typical of individuals implementing FFT. Another limitation was that the families and youth who received FFT services were not included in this study. The families and youth are key contributors in the implementation process and are important stakeholders in transporting evidence-based practices into community settings. Also, due to the nature of the small sample size, the findings of the study are not generalizable; however, it can be reasonably said that other mental health and human services organizations may have similar issues with the adoption and implementation of FFT.

The next study by Zazzali et al. (2008) points to the individual and organization factors associated with the adoption of FFT in mental health organizations and what particular organizational factors (e.g. resources, IT support, and organizational culture) are critical for FFT's successful implementation among therapists in a New York State Child and Family Mental Health Services Organization. Zazalli et al. (2008) conducted semi-structured voluntary interviews at 13 agencies that recently adopted FFT from September 2003 to March 2004. The respondents were all administrators in their organizations. The team conducted 14 interviews with 15 administrators with one of the 
interviews that consisted of two administrators at one time.

The interview questions pertained to the drivers of adoption (e.g. reasons behind adoption of FFT), and the organizational barriers and facilitators (e.g. champions and leaders, technology, resources) during FFT implementation. All interviews were taperecorded and then transcribed. The qualitative data analysis software package N6 was used to analyze the transcribed data. A priori coding schemes were developed and used to code the 14 interviews as well as other coding themes that were not a priori were developed and used as they became apparent from reading the transcripts. A total of five major coding headings were used (drivers of adoption, barriers and facilitators to implementation, perceived success of FFT, and continuance of the FFT program).

The results of the Zazzali et al. (2008) study were separated into reasons of FFT adoption and the organizational barriers and the facilitators during FFT implementation. For the adoption of FFT, the authors decided there were internal and external reasons for the adoption of FFT. The internal explanation for the adoption of FFT included that FFT fit the goals and the philosophy of the organization. The external explanation for adopting FFT included the "institutional effect," meaning the mental health organization adopted FFT because other organizations were offering FFT services. Organizations that were adopting FFT felt pressure to conform to other agencies to ensure their legitimacy. Also, when county mental health organizations offered funding there was evidence that organizations that adopted FFT received funding. 
Many organizations adopted FFT because it fit the organization's goals and philosophy. However, these organizations also identified the many barriers to implementing FFT as well as other reasons for adopting FFT. The sites that had fairly well IT infrastructures facilitated FFT's implementation given the heavy documentation requirements of FFT. In addition, those organizations that said FFT "fit" were organizations that preferred change and were innovative making FFT adoption easy. Those organizations that did not have IT resources or staff skilled at using computers prior to implementation fell behind in documentation. Other barriers that were most often cited included staff turnover. Staff turnover required additional training and indicated that the lost revenue during training was a serious issue for the organization. Also, most respondents indicated that FFT documentation requirements of the model and the expected fidelity to the model were burdensome, particularly when the documentation combined with existing clinic and/or county paperwork.

Zazalli et al. (2008) results also revealed that clinicians were discouraged with some features of the model. For instance, clinicians wanted to refer those with drug and mental health issues to other services, even though it deviated from the FFT model. Clinicians believed that it was clinically called for to refer youth to mental health therapists to treat youth for depression and anxiety, yet it was discouraged by FFT because it is conceived as not necessary within the model. Other clinicians took issue with the requirement that all family members attend the family sessions and were not allowed to meet 
with the youth alone. Other respondents indicated that FFT was perceived to be different from what they thought it to be prior to implementation. FFT required much more time and financial commitment than expected. Two respondents indicated that the external stakeholders, such as the family court had unrealistic expectations of what the model could accomplish in a limited period of time.

The limitations of the Zazalli et al. (2008) study included a small sample size of administrators and the study did not include the therapists delivering FFT and the familie receiving FFT. Also, the findings were based on 15 interviews at 13 different organizations. Therefore, the ability to generalize beyond the sample was limited.

The above studies examined the individual and organizational issues that assist and preclude program adoption and fidelity of FFT in human services organizations. Next, this literature review provides studies on the organizational and individual-level factors that assist and preclude program fidelity of evidence-based programs in juvenile justice organizations. The following studies in juvenile justice programs reveal similar organizational issues similarly found among counselors and therapists in human service organizations in implementing family-based models such as FFT.

Implementation of Evidence-based Practices in Juvenile Community Corrections 
and in Mental Health Services

With the quest for outcomes studies to determine what does work in the treatment and prevention of crime and delinquency, program evaluation studies in the human services field have examined the individual and organizational-level factors that assist the ease of implementation of programs (Glisson, 2002; 2007; Hemelgarn et al., 2006). What is missing in the literature are discussions that examine these same individual and organizational-level factors found in juvenile community corrections. To date few studies have examined individual and organizational-level phenomena that affect program fidelity and sustainability at the juvenile community corrections level (Farrell et al., 2011; Mihalic, \& Irwin, 2003; Mihalec et al., 2004).

To fill this gap a study conducted by Farrell et al. (2011) examined the relationships between the organizational and individual-level factors that facilitate the use of current best practices in a juvenile correctional agency as well as create barriers. Four primary organizational characteristics were examined — organizational climate, supervisory leadership, staff cynicism, and interagency coordination in one eastern state in 33 field offices. A survey was administered in all 33 field offices to 393 probation officers, social workers, resource specialists, addictions specialists, investigators, court liaisons, and supervisors to assess treatment planning, referrals, and all the offices' monitoring practices. Research staff described the project, handed out the paper-and-pencil surveys to field office staff, and remained available during the survey session to answer any ques- 
tions. Overall, 554 staff members were eligible to complete the survey; of these, 445 (82\%) completed the surveys, including 353 who finished it during the office visit and 92 who mailed in a completed survey. The response rate was the same across 32 offices; in 1 office, only a $50 \%$ response rate was achieved. The lower response rate was in an office that was substantially larger than the typical field office. The overall response rate for the state excluding the one office with only a $59 \%$ response was $89 \%$ (396 of 446 eligible staff members).

For the analysis, organizational measures and responses were analyzed at the individual level and office-level (aggregated at the unit) as the independent variables. Specifically, at the individual level, the authors measured staff cynicism for change, organizational climate, and perceptions of their supervisor's leadership. At the office level they measured integration with the courts, the state mental health agency, and integration with community-based providers. Staff cynicism for change was measured using a 5-item scale that indicated the extent to which the staff member is pessimistic about the organization's ability to change procedures or improve. Participants were asked to respond on a 5-point Likert-type scale ranging from 1 (strongly disagree) to 5 (strongly agree) to items such as "efforts to make improvements in this agency usually fail." Office climate reflects the degree to which individuals view their organization as open to change and supportive of new ideas. This variable was assessed using 4 sub scales, Management Focus on Performance, Support for Development, Support for Innovation, and 
Communication. The Management Focus on Performance sub scale indicates the extent to which staff is provided with performance measures and the use of performance measures to improve officers' practices. The Support for Staff Development sub scale measures the extent to which staff is provided with information or opportunities to learn new skills related to their practices. The support for Innovation sub scale reflects the extent to which management promotes and encourages staff to be innovative. Finally, the Communication sub scale assessed the effectiveness of both formal and informal communication channels. Each of these sub scales were strongly correlated with one another; thus they were combined to create a single measure indicating a favorable organizational climate. Leadership was assessed using a scale that consisted of 8 items that indicated the extent to which a staff member feels his or her supervisor "leads by example" and "provides well-defined performance goals and objectives."

The control variables were controls for personal factors: education level, age, and training. The level of education and attitude toward change should affect whether individuals adopt innovative practices. Research in addiction treatment suggests that staff qualifications significantly affect the intent to adopt different type of evidence-based practices; for example, staff with graduate degrees are more likely to adopt innovations (Aarons, 2004). At the staff level, the study controlled for gender, educational level, job tenure, and whether he or she works in a special unit that supervises specific types of youth, such as females or sex offenders. Also the study controlled for geographic location 
of the field office. Location was operationalized as urban, suburban, large town, or rural on the basis of its population size, population density, and proximity to metropolitan areas.

Included in the analysis was the dependent variable, the Juvenile Service-Oriented Practice Scale (JSOP). The JSOP is a summated scale of 10 practice measures that exemplify core components of effective youth supervision. Each item in the JSOP represents a practice that probation and aftercare officers may use with their caseloads. This variable includes the use of screening and assessment tools by probation officers, program referral considerations (consider at least 5 of 7 factors when making referral decisions), treatment plan components (incorporates at least 3 of 4 procedures with most of the youth), youth involvement (includes youth in treatment planning most of the time,) family involvement in the treatment and planning process (at least 4 of 6 procedures with most of the youth), improve treatment compliance (uses at least 6 of 9 procedures with most of the youth).

The analysis examined the responses of $332(84.5 \%)$ probation officers and $61(15.5 \%)$ supervisors. To examine the relationships between the individual staff characteristics, and organizational measures, Pearson correlation analysis were conducted to examine zero-order correlations between all staff-related measures, organizational measures, and the JSOP. Then, regression analysis were conducted to examine the associations of individual and organizational level predictor variables with the JSOP. 
Because staff members were nested within offices, hierarchical linear modeling analysis were conducted to control for potential correlations between responses within offices. When analyzed as independent observations, hierarchically nested data violate important assumptions of multiple regression models, typically resulting in downwardly biased standard errors and inflated Type I error rates.

Several variables are significantly related to the dependent variable, the JSOP scores. In terms of staff-level characteristics, working in a special unit $(r=.171, p<.01)$, organizational climate $(r=.106, p=.01)$, and supervisory leadership $(r=.162, p<.01)$ are significantly and positively related to the use of service-oriented practices. On the other hand, the relationship between staff cynicism for change and the JSOP is negative and significant $(r=-.195), p<.001)$. Gender, age, a graduate degree, and job tenure are not correlated with use of these supervision practices. At the office level, all three measures of agency integration are positively associated with JSOP scores (courts, $r=$. 177, $p<.01$ SMHA, $r=.168, \mathrm{p}<.01$; CBSPs, $r=.288, \mathrm{p}<.001)$. For organizational measures, cynicism for change has a moderate to high negative relationship with office climate $(r=-.598)$. Also, office climate has a moderate to high positive relationship with supervisory leadership $(r=.505)$.

Results from the hierarchical linear modeling indicated that several staff and office-level factors exert direct effects on the JSOP. At the office-level location was significantly related to the JSOP, where staff in urban offices had significantly higher 
JSOP scores relative those in suburban areas. In addition, staff in offices that have greater integration with community-based services providers were more likely to use serviceoriented practices. At the staff-level of analysis those who worked in a special unit and reported higher ratings of their supervisor's leadership had significantly higher scores on the JSOP. And higher reports of cynicism for change were significantly related to lower scores on the JSOP. Staff gender, education, and job tenure were not related to the JSOP scores. Finally, perceptions of organizational climate were not significantly related to JSOP scores. Overall, the results revealed that lower levels of staff cynicism for change, more favorable perceptions of supervisory leadership, and greater integration with community-based service providers were significantly related to greater use of serviceoriented practices among supervision staff.

According to Farrell et al. (2011) the study's limitations is that the JSOP measure is limited in that it did not encompass some important supervision components and principles, such as motivational interviewing and a strength-based focus with youth and their families. Furthermore, this measure was based on staff-reported practices, and more objective measurements techniques may yield lower (or higher) levels of implementation. This study was also not able to address several factors at the organizational and individual levels that have been shown to affect staff behaviors in other human service organizations, for the study was not able to assess the organizational culture, the training and the funding resources. Further, the data used was cross-sectional; thus causal 
direction of the relationship between organizational factors and use of service-oriented practices was not assessed. Causal relationships can be only assessed with longitudinal data or experimental methods.

The next study conducted by Aarons \& Sawitzky (2006) examined the association of organizational culture and climate with attitudes toward adopting evidence-based practices in mental health organizations that provide services to youth and families. Previous studies have indicated that providers working with youth with more positive culture's have more positive attitudes toward the adoption of evidence-based practices, whereas those providers who worked negative cultures endorsed more negative attitudes toward adoption of evidence-based practices. Moreover, organizational culture has also been shown to impact organizational change by facilitating or hindering the change process during implementation of evidence-based practices. Participants in the study consisted of 301 public sector mental health service providers from 49 programs that provided mental health services for youths and families.

The participants answered a series of surveys at their work sites. Prior to administering the surveys a program manager was contacted at each site and the study was described in detail. The project coordinator or a trained research assistant administered the surveys and were available during the survey sessions to answer any questions that arose. A few surveys were left for completion for providers who were not in attendance at the survey sessions. Such surveys were either mailed back in a prepaid 
envelope or picked up by a research assistant. Participants received a verbal and a written description of the study and informed consent was obtained prior to the survey. The study participants were clinical and case management mental health service providers who participated in a larger study of organizational issues affecting mental health service for children and adolescent and their families in San Diego County, California. Eighty percent of respondents were full-time employees, and their primary disciplines included marriage and family therapy, social psychology, psychiatry, and psychology.

Participants answered two surveys that incorporated questions regarding provider demographics including provider age, sex, education level, professional status (intern vs. professional), job tenure, and questions regarding their organization's organizational characteristics. The first survey, the Evidence-Based Practice Attitude Scale (EBPAS) (Aarons, 2004), was used to assess mental health provider attitudes toward evidencebased practices. The EBPAS is a brief 15-item measure with four sub scales assessing attitudes toward adoption of evidence-based practices and one overall total scale. The EBPAS sub scales represent four theoretically derived dimensions of attitudes toward adoption of evidence-based practices: appeal, requirements, openness, and divergence.

The second survey, the Children's Services Survey (Glisson, 2002) was used to assess both organizational culture and climate. The organizational culture scales were derived from the Organizational Culture Inventory (Cooke \& Rousseau, 1988) and adapted for use in mental health services (Glisson \& James, 2002). The organizational 
climate scales in the Children Services Survey were based on organizational studies in diverse workplace settings (Cooke \& Rousseau, 1988). The organizational climate sub scales assess dimensions of depersonalization, emotional exhaustion, and role conflict and has been validated in previous work with reliabilities ranging from 0.69 to 0.92 (Glisson \& James, 2002). Lower scores on these scales indicate more positive climate and higher scores indicate a more negative organizational climate.

For the analysis Pearson product-moment correlation analysis were first conducted to examine zero-order correlations between the independent variables that included organizational and individual-level predictors, and the dependent variables (e.g. EBPAS scores representing attitudes toward evidence-based practices). Next, the study conducted five regression analyses to examine the associations of individual and organizational-level with each of the four EBPAS sub scales and the EBPAS total scale. Because providers were nested within mental health programs resulting in potential dependency of responses within program, multilevel hierarchical linear model analyses were conducted to control for the effects of the nested data structure.

The results suggested that a positive culture and climate are conducive for adoption of evidenced-based practices. A constructive culture was significantly positively associated with the Appeals scale scores $(r=.133)$ and Total $(r=.198)$ scales. The EBPAS Appeal scale found that providers who worked in constructive cultures and those with higher educational attainment endorsed positive attitudes toward adopting EBPS. 
The EBPAS openness scale regression analysis showed that constructive culture was significantly positively associated with the openness scale scores. This indicated that providers working in programs with more constructive cultures endorsed greater openness toward adoption of evidence-based practices. Additionally, age was significantly positively associated and job tenure was significantly negatively associated with openness. These results indicated that, although older providers were more open to adoption of evidence-based practices, those who worked at their program for longer periods scored lower on Openness to evidence-based practices. The EBPAS Requirements scale analysis showed that there were no statistically significant associations of organizational or individual-level predictors with the requirements scale scores. However, there were marginal effects suggesting that providers working in organizations with more defensive cultures and those with higher educational attainment were more likely to endorse adopting an EBP, given the requirement to do so. The EBPAS divergence scale regression analysis showed that organizational climate and provider age were both significantly positively associated with divergence scale scores. This indicated that providers. Particularly older providers, working in organizations with more negative (e.g. demoralizing) organizational climates endorsed a greater perceived divergence between EBP and their usual practice. Finally, EBPAS total scale regression analysis showed that constructive culture and intern status were both significantly positively associated with EBPAS total scale scores. These results generally support our 
hypothesis that providers working in organizations with more constructive cultures and providers earlier in their professional careers were more open to adopting evidence-based practices. Although older providers were more open to adoption of evidence-based practices, those who worked at their program for longer periods scored lower on the openness scale. The main finding of this study was that organizational culture and climate are associated with mental health service providers' attitudes toward adoption of evidence-based practices.

Some limitations of this work should be noted. First, because the EBPAS is a relatively recently developed scale, more studies should be conducted to support its reliability and validity. Second, five regression models were estimated; thus, inflation of Type I error rates could be a factor in this set of analyses. This study involved public sector mental health providers, and results may not generalize to other contexts or provider groups. Further this analysis was exploratory and additional studies including outcomes studies should be conducted.

Nevertheless, the implications of the present study are relevant for organized care settings outside of the specialty mental health care sector. For example, there is ongoing work in implementation of evidence-based practices in child welfare, social services, corrections, schools, and primary care settings, for all have organizational contexts that may facilitate or hinder implementation of innovation. As such, a better understanding of provider attitude in relation to organizational context should be considered when 
implementing evidence-based practices. The present work adds to the evidence that organizational context; including culture and climate are important considerations in the implementation of evidence-based practices. Essentially, fostering cultures that promote adoption of innovation may help to improve the ease and success of moving evidencebased practices in community settings.

Summary of Literature Review

The overall key points of the previous six studies in this literature review indicate that organizational and individual-level contexts must be addressed for the successful implementation and sustainability of evidence-based programs. Gordon \& Arbuthnot's (1988) study suggested that the lack of organizational and individual level (cynicism) support or support from the bench and management led to the eventual demise of FFT. However, individual-level factors, such as the warmth and empathy of the therapists facilitated training and FFT's implementation among the Atlanta juvenile probation officers. Despite the programs demise, the study demonstrated that probation officers could be trained in this community-based model. Similarly, Rowland's (2007) study on model adherence and competence of juvenile parole officers suggested that juvenile parole officers could be trained in a family-based intervention. Moreover, Rowland's (2007) suggested that the model adherence and competence of the juvenile parole officers was a moderator of youth outcomes. However, Rowland's study did not examine the individual and organizational-level factors that assist or preclude program fidelity, nor the 
advantages and disadvantages of a family-based intervention.

Duncan et al.'s (2011) study addressed the advantages and disadvantages of FFT. In in this present study, many of the advantages and disadvantages of FFPS as a case management model among experienced by the Multnomah County juvenile court counselors were similar to the experiences of family therapists delivering FFT to their youth and family clients in Duncan et al.'s (2011) study examining therapists delivering FFT.

As for the organizational and individual-level characteristics, such as a positive culture and climate, are instrumental in the implementation and sustainability of programs. Zazalli et al.'s (2008) study suggested that organizational characteristics that include an organization being innovative and believing in FFT approaches are necessary for the successful adoption and implementation of FFT. Farrell et al.'s (2011) study suggested that specific organizational and individual-level characteristics, such as favorable perceptions of supervisory leadership and lower levels of staff cynicism were significantly related to greater use of service-oriented practices among juvenile probation staff. Finally, Aaron and Sawitzky's (2006) study suggested that mental health providers adoption of an evidence-based practice are associated with a positive culture and climate. Positive attitudes among mental health provides toward adopting evidence-based practices, and poor climate (burnout, cynicism) among providers was associated with divergence from adopting and implementing evidence-based practices. 


\section{CHAPTER THREE}

\section{Method and Design}

This case study examined the Juvenile Court Counselors', the Community Justice Managers', and Senior Managers' experiences with the implementation of FFPS core components and their perceptions on the advantages and disadvantages of FFPS as a case management model at Multnomah County Juvenile Services Division in Portland, Oregon. Examining and assessing the participants' perceptions on the individual and organizational factors that facilitate and preclude program fidelity will assist informing change necessary to improve FFPS program fidelity. Further, studying their experiences may help other community corrections professionals to have a better understanding on the individual and organizational changes that better facilitate FFPS's implementation and sustainability.

In evaluation research, a case study allows an investigator to explore, describe, explain, and determine causal links of phenomena occurring among individuals or in an organization in greater depth (Patton, 2002; Yin, 2008). Yin (2008) explains that case studies answer the research questions "how" and "why" a program works or "how" and "why" an organization implements a program. Case studies call for direct observation of events being studied and interviews of the participants involved. To delve into greater depth, the case study allows for and recommends many methods of data collection-interviews, observations, documents, media, artifacts—-by the investigator or a 
participant/observer to assist corroboration, increasing the validity of the study (Yin, 2008).

This case study's goal is to provide a better understanding of the Juvenile Court Counselors', Community Justice Officers', and Senior Managers' experiences and their understandings of the implementation and delivery of the FFPS model in their own words. In this study, interviewing the Juvenile Probation Officers and soliciting their understandings, experiences, attitudes, and perceptions with FFPS provides a more indepth examination of the program's implementation and the many organizational and individual-level factors that assist or preclude program fidelity (Greene, 1998; Sharma, 2004; Slayton \& Llosa, 2005). Without a qualitative evaluation that assesses fidelity to the model during implementation, outcome results may be difficult to interpret (Altschuler \& Armstrong, 2002). Altschuler \& Armstrong (2002) warn that even the bestdesigned and documented model can fail due to implementation problems.

This study addresses the following research questions:

1. What are the Juvenile Court Counselors', Community Justice Managers', and Senior Managers' opinions and experiences with the implementation of FFPS?

2. What are the Juvenile Court Counselors', the Community Justice Managers', and the Senior Manager's experiences of what individual and organizational factors that assist or impede the implementation of FFPS core components? 
3. What are the Juvenile Court Counselors', the Community Justice Managers', and the Senior Managers' opinions on the advantages and disadvantages of FFPS as a case management model?

\section{Researcher Background}

The Juvenile Court Counselors, the Community Justice Managers, and Senior Managers were familiar with this researcher who served as an assistant Juvenile Court Counselor in the Sex Offender and Female Gender Units for two years for 20 to 25 hours a week. This investigator also worked with other Juvenile Court Counselors in the RISE and FSU unit. Further this investigator served as a co-evaluator for the Multnomah County Department of Community Justice in two program evaluations prior to the FFPS study. This researcher had received training in FFPS and has observed and participated in delivering FFPS to youth and families with the Juvenile Court Counselors located in the Sex Offender and Gender Units. Because this researcher worked with the Juvenile Court Counselors this researcher has an understanding of the culture and climate of Multnomah County Juvenile Services Division.

According to Marshall and Rossman (2011) and Bonner and Tolhurst (2002), the role this researcher has as a participant observer is very common in case study qualitative research. Essentially, the participant observer is already a "native" or "insider" within this group (Atkinson \& Hammersley, 1998). Yin (2008) writes that participant observers have a greater understanding and access to phenomena, and in the past the technique has been 
used in anthropological studies of different cultural or social groups. Bonner and Tolhurst (2002) state that being a participant observer gives the researcher the ability to understand the participants' thoughts, feelings, and actions in greater detail. Furthermore because the researcher is considered a group member, there will be little disruption in the normal activities of the Juvenile Court Counselors, the Community Justice Managers, and Senior Managers at the site.

\section{Setting}

This study was conducted at Donald E. Long Juvenile Services Division building located at 1401 N.E. 68th Avenue, Portland, Oregon 97201. This large complex built in 1995 houses the Detention Center, the secure residential alcohol and drug treatment center, the courthouse, the cafeteria, the probation offices, the accountability program center, data services, and the state Oregon Youth Authority probation and parole offices.

\section{Participants}

This case study utilized criterion sampling as its procedure. Criterion sampling involves selecting cases that meet predetermined criteria of importance (Patton, 2002). The criteria required that the Juvenile Court Counselors, the Community Justice Managers, and the Senior Managers be trained in FFPS. During this study not all Juvenile Court Counselors were trained in FFPS. Non-field Juvenile Court Counselors who worked in other departments not responsible for FFPS delivery were not trained. 
Out of a total of 23 FFPS trained, 17 FFPS trained participated in the study. All 17 of the participants all have offices located at the Donald E. Long Juvenile Services Division Complex. The participants included two Senior Managers, four Community Justice Managers, and 11 Juvenile Court Counselors (out of total of 16 JCCs trained in FFPS at the time of the study). Participants were recruited at the site by requesting their participation via email or asking them verbally in their offices. All staff interviewed in this study came from diverse ethnic backgrounds and were between the ages of 35 and 65. Five were African American; one was a Pacific Islander; three indicated their family origins were from Southeast Asia; and eight were Caucasian. There were four men and 14 women. All had at least 10 or more years' experience working with youth and families. Most staff acquired at least a four-year degree and a master's degrees in criminal justice, education, social work, and counseling.

The Juvenile Court Counselors work in five specialized units and are responsible for delivering FFPS. At JSD these five units serve approximately 300 to 340 youth. These units include the Sex Offender Unit, the Resource Intervention Services to Empower (RISE) unit, the Female Gender Unit, the Family Services Unit, and the Informal Unit. The descriptions of the five units:

1. The Resource Intervention Services to Empower (RISE) Unit provides probation supervision to high risk, gang-involved offenders using strategies that are tailored to each youth's issues, strengths, needs, culture and environmental influences. 
This unit partners with the police, Adult Parole/Probation Officers, and the community to gather intelligence that helps dismantle gang activity.

2. The Sex Offender Unit supervises youth who are adjudicated for sex offenses. These youth are predominantly males 12-18 years of age. They have appeared before a judge or referee and received special court ordered treatment conditions and sanctions pertaining to their sexual offending behavior.

3. The Female Gender Unit provides supervision to medium and high-risk females on probation. This program partners with the Department of Human Services, Wrap Around Oregon, and the Oregon Youth Authority to address the needs of these young women. This program works together to handle the assessment, adjudication, placement, case planning, and treatment services that are tailored to the specific needs of young women.

4. The goal of the Family Services Unit is to enhance community safety by reducing inter-generational criminal behavior by promoting positive change in offenders, and working with a family's relationships that affect recidivism and compliance with the conditions of supervision. Juvenile Court Counselors in the unit work in partnership with other service providers, including community health nurses and other human services agencies.

5. The Informal Unit goal is to work with medium to high-risk youth who have not been formally adjudicated through the criminal justice system. These youth are 
mostly first-time offenders and to keep youth from penetrating into the system, team members work with youth and families referring them to intervention services. (At the time of the study, this unit was delivering FFPS to youth and families. They are no longer delivering FFPS services).

\section{Data Collection}

Data collection began July 7, 2012 and ended October 5, 2012. The data collection included semi-structured interviews of the Juvenile Court Counselors, the Community Justice Managers, the Senior Managers, and observations of FFPS consultation sessions (See Appendices). During the FFPS observations this investigator took written notes and completed the FFPS observation checklist (created by this investigator; see Appendices). Fifteen observation notes and checklists and 17 semistructured interviews were generated. Interviews of the Juvenile Court Counselors, the Community Justice Managers, and the Senior Managers took from 45 to 90 minutes. The interviews were conducted in conference rooms, in restaurants, in parks, in staff offices, and in county vehicles while out in the field. Ten of the $11 \mathrm{JCC}$ interviews were recorded and then transcribed. Written notes were taken during the Community Justice Managers' and the Senior Managers' interviews.

Observations and interviewing are the most common form of qualitative case studies and the goal of qualitative data analysis is to uncover emerging themes, patterns, concepts, insights, and understandings (Patton, 2002). Interviewing provides a rich 
exploration of the Juvenile Court Counselors', the Community Justice Managers' and Senior Managers' experiences and understandings of FFPS implementation. So too, Marshall and Rossman (2011) and Denzin (1989) state that interviewing is the best method for gathering an individual's lived experiences and their interpretation of those experiences in their lives. The semi-structured interview questions were created by this investigator and were derived from program evaluation studies that examined counselors and family therapists delivering family-based interventions (e.g. FFT) and intervention programs in corrections and human services (Aarons \& Sawitzky, 2006; Duncan et al., 2011; Farrell, et al., 2011; Gendreau, et al., 1999; 2001; Glisson, 2002; Sharma, 2004, Slayton \& Llosa, 2005). The interviews were semi-structured to allow for possible probes that would allow the researcher to to ask more questions if needed to increase exploration of interviewees' understanding.

Qualitative strategies recommend that data collection continue until saturation is reached (Morse, 1994). Saturation refers to the idea that once the researcher finds that further interviews or observations are not adding additional relevant information then there is no need to continue interviewing additional participants (Denzin \& Lincoln, 1994; Marshall \& Rossman, 2011). Saturation of all 17 interviews was not reached. Out of the 23 staff that received FFPS training, 17 participated in the study. Most of the study participants, especially the Juvenile Court Counselors, provided similar information, but each interviewee had unique insights, experiences, and understandings. 
Observations of FFPS consultation meetings in all five units ranged from 45 to 120 minutes in conference rooms. This researcher was a non-participant observer (Adler \& Adler, 1994) during FFPS consultation sessions and sat to the side or the back of the room away from the conference tables in order to not interfere with the meeting. Written notes and the observation checklist (See Appendices) were utilized to gather information. The observation checklist was designed by this researcher and was derived from program evaluation and implementation studies found in the human services literature (see also Duncan et al., 2011; Mykota, 2008; Sharma, 2004, Slayton \& Llosa, 2005). The checklist was designed to keep track of discussions related to FFPS components and to document observations of resistance, burnout, and cynicism, as well as observations of positive worker morale or no indications of resistance. There were no previous validated instruments, such as an FFPS observation checklist that covered FFPS implementation among community corrections professionals. Saturation was achieved for the FFPS consultation sessions in all five units.

According to Patton (2002), observational study has four strengths that should be noted. First, direct personal contact gives the investigator the ability to better understand and capture the context within which people interact. Understanding the entire environment and all the simultaneous behaviors whether verbal or non-verbal allows the investigator to grasp the individual and organizational-level dynamics occurring during meetings. Second, being on-site the observer can discover inductively and not rely on 
prior biases and conceptualizations. Third, the investigator can see things that interviewees may not be aware of because certain practices are routinized or are not visible to the interviewee. One of the highlights of the participant observer is that the investigator can notice things that no one else noticed or thought to mention during interviews. For example, observations of the FFPS consultation sessions served to corroborate whether the Juvenile Court Counselors and Community Justice Managers were learning the model. Observations of the meetings that included discussions on remaining barriers to FFPS delivery served to provide critical information. Fourth, with direct observation the investigator can observe occurrences and behaviors that interviewees may not have wanted to discuss. For example, most of the Juvenile Court Counselors avoided interview probes regarding worker morale, resistance, cynicism, burnout, and union issues. A caveat is that field observers have selective perceptions; however, Patton (2002) argues that investigators can make their own perceptions as part of the data, combined with discipline, training, and self-awareness, observers can arrive at a more comprehensive view of the setting. Another caveat is that sometimes people behave differently when they are being observed (Posavac \& Carey, 2007).

\section{Analysis}

According to Merriam (2009), qualitative data analysis and collection occur simultaneously. Meaning, the findings from previous observations and transcripts guide the researcher to test theories and ideas in subsequent interviews and observations. During 
each interview and observation, the investigator analyzes, compares, and organizes all the data. After all the data is transcribed, the researcher searchers for patterns searching for themes. This is called conducting a thematic analysis which is the most common form of analysis in qualitative studies (Charmaz, 2006; Cresswell \& Clark, 2007; Denzin \& Lincoln, 1994; Patton, 2002; Shank, 2006). First the researcher coalesces the data that have commonalities into codes and then puts those codes into conceptual categories (Marshall \& Rossman, 2011). After the codes are created and put into categories, the next level of abstraction involves axial coding (Corbin \& Strauss, 2007). Axial coding is the grouping of open codes so that their categories (and properties) relate to each other in some analytical way. The guiding question in this step is, "How are these categories related?" "Might they reflect a causal chain?" "Do they interact?" "Are they instances of a broader context?" The coding method was used to organize the data into a limited number of themes and issues around the research questions. Both the codes and the categories for this study were derived inductively and deductively (using program evaluation literature). Boyatzis (1998) provides five guidelines of a good thematic code to increase the reliability and validity of a theme (p. 31).

1. A label (i.e. name)

2. A definition of what the theme concerns

3. A description of how to know when the theme occurs 
4. A description of any qualifications or exclusions to the identification of the theme.

5. Examples, both positive and negative, to eliminate possible confusion when looking for the theme.

From these coded categories, the investigator looks for patterns and combines these patterns into themes consistently to ensure data reliability (Boyatzis, 1998; Patton, 2002). Boyatzis (1998) states that a theme represents a level of patterned response or meaning from the data that is related to the research questions at hand. According to Braun and Clark (2006, p. 82), "A theme captures something important about the data in relation to the research question, and represents some level of patterned response or meaning within the data set." Themes are generated inductively and deductively (Boyatzis, 1998) similar to coding. Boyatzis (1998) explains that in an inductive approach the themes identified are strongly linked to the data that were generated from the study and similar to coding. Crabtree (1999) states these themes may also be theory-driven and may be limited to preconceived frameworks (for this study, the constructivist approach). The program evaluation and implementation literature in criminal justice and human services provided the framework for the themes. For instance, categories that were generated based on the evaluation and implementation literature found in human services and criminal justice organizations included facilitators and barriers to program fidelity, implementation, pros and cons of family-based interventions, organization and individual-level 
factors (e.g. buy-in, resistance, technology, resources, culture, leadership, and training, and climate). Furthermore, Braun and Clarke (2006) state that frequent themes are chosen mostly; ideally, a theme occurs numerous times. However, a higher frequency does not necessarily mean that the theme is more important to understanding participants' experiences and the purposes of the study (Braun \& Clark, 2006). Ultimately, it is the researcher's judgment that chooses which themes and quotations increase understanding of the phenomena being study.

\section{Limitations of the Design}

Qualitative studies that incorporate observations, documentation, interviews as the primary source for data collection have their own limitations in method and design. This case study had three limitations in the design and method. First, whenever an evaluator observes participants in a setting, it is likely that the presence of the evaluator will change the way that those being observed will act (Posavac \& Carey, 2007; Schwartz \& Schwartz, 1955). However, because this investigator spent two years with the participants, participants viewed this investigator as a colleague rather than a researcher, and this researcher noticed few alterations in participant behavior when this researcher was on site.

Second, for evaluation and case studies it is routine practice to use the observations, documents, and interviews to corroborate and triangulate in order to increase internal validity. Denzin (1989b) argues that no single method, theory, or observer can cap- 
ture all that is relevant or important in reality. Corroboration and sometimes called, triangulation, is a method used to increase validity of qualitative studies (Denzin, 1989b; Yin, 2008). Triangulation is defined as a method used in qualitative research that involves cross-checking multiple data sources and collection procedures to evaluate the extent to which all evidence converges (Denzin 1989b; Marshall \& Rossman, 2011; Yin, 2008). . Yin (2008) adds to this concept by stating that qualitative analysis of text is often supplemented with other sources of information to satisfy the principle of triangulation and increase trust in the validity of the study's conclusions. It would not be uncommon, for example, to analyze transcribed interviews along with observational field notes and documents authored by the respondents themselves. However, for this evaluation, written documentation in the form of Juvenile Justice Information System case notes and FFPS family service plans and FFPS notes were not included in the data collection because these documents were rarely current. Instead FFPS consultation sessions assisted corroboration and triangulation of recorded transcripts and written interview notes.

Third, being a participant observer or an "insider" provides an excellent opportunity to observe the integrity of program implementation as well as provide a better understanding of the opinions and experiences of the Juvenile Court Counselors, Community Justice Managers, and the Senior Managers. While there are some benefits of the participant observer's role, it also has limitations. Because of an "insiders" relationship with the participants, the risk of "going native" (Gold, 1958) and "over- 
rapport" with the subjects (Miller, 1952) is increased. Both of these suggest that the qualitative researcher could be unable to maintain objectivity.

\section{Researcher Trustworthiness}

Trustworthiness pertains to how much the evaluator or researcher has adhered to procedures specific to the chosen method and design that answer the research questions (Sharma, 2004). Trustworthiness in qualitative studies is referred to as 'validity' in quantitative studies. Trustworthiness is an essential component of qualitative research and according to Lincoln and Guba (1985) there are four criteria: credibility, transferability, dependability, and confirmability. For credibility, Lincoln and Guba (1985) state that in order to ensure credibility the researcher must spend a prolonged period of time at the site. A prolonged time at the site allows the researcher to become more oriented to the situation so that the context is appreciated and understood. In this case, the researcher was at the site for two years with the last four months devoted to conducting the FFPS study. Credibility will also be ensured by the researcher by allowing the participants to do member checks (Marshall \& Rossman, 2011). Member checks require that after the researcher writes their conclusion, he or she asks the participants if the summaries are correct and if the participant has any further insights or corrections to make. This step ensures that the data is grounded in the participants' understandings and experiences and not the researcher's understandings and biases (Marshall \& Rossman, 2011). Follow up member checks for half of the study participants were over the phone or 
via email after the study were conducted. Other participants did not respond to member checks.

Lincoln and Guba's (1985) second criteria of trustworthiness is transferability. Transferability is ensuring that the findings have applicability in other contexts. To do this, the researcher will interview and probe for thick descriptions of the Juvenile Probation Officers experiences and understandings of FFPS implementation. By describing a phenomenon in sufficient detail one can begin to evaluate the extent to which the conclusions drawn are transferable to other times, settings, situations, and people (Lincoln \& Guba, 1985; Sharma, 2004). It has been duly noted by this researcher that transferability receives criticism from quantitative researchers who argue that qualitative research is not generalizable or transferable.

Lincoln and Guba's (1985) third component of qualitative research is dependability, which is showing that the findings are consistent and could be repeated in other settings with similar participants. To ensure the data and analysis, this researcher will take Lincoln and Guba's (1985) suggestion of utilizing external audits, which involves having a researcher not involved in the research process examine both the process and product of the research study. The purpose is to evaluate the accuracy and determine whether or not the findings, interpretations, and conclusions are supported by the data.

Finally, Lincoln and Guba's (1985) fourth component of qualitative research is confirmability. Confirmability is meant to guarantee the degree to which the findings of a 
study are shaped by the respondents and not by the researcher's own bias and interpretations. According to Sharma (2004) confirmability is difficult to achieve in qualitative studies because it depends on values and is subjective. Boyatizis (1998) states that one of the major threats in conducting thematic analysis is the researcher contaminating the findings by projecting or attributing to the study their own emotions, values, and attitudes. Preventing contamination of projection is helped by sticking close to the raw written notes and transcripts, and code the raw data consistently. Lincoln and Guba (1985) also suggest that the researcher develop a reflexive journal. This type of journal has the researcher make regular entries about each step in the research process. In these entries, the researcher records methodological decisions and the reasons for them as well as the logistics of the study. In addition the researcher records reflections upon what is happening in terms of his or her own values and interests and possibly develops an awareness of his or her own biases and opinions. This diary keeping kept further of this type is often very private and cathartic (Lincoln \& Guba, 1985).

\section{CHAPTER FOUR}

Results 


\section{Introduction}

This case study examined the Juvenile Court Counselors' (JCCs), the Community Justice Managers'(CJMs), and Senior Managers' self-reports on their experiences with the implementation of FFPS core components and their perceptions on the advantages and disadvantages of FFPS as a case management model at Multnomah County Juvenile Services Division in Portland, Oregon. Observing and examining the self-reports of the participants' experiences with FFPS and the participants' perceptions on the individual and organizational factors that facilitate and preclude program fidelity will assist informing change necessary to improve FFPS program fidelity. Further, studying their experiences may help other community corrections professionals to have a better understanding on the individual and organizational-level factors that are necessary to facilitate successful implementation and sustainability of FFPS in other juvenile justice agencies.

The core components of FFPS include: JCC delivery of FFPS to county youth and families, JCCs complete FFPS service and case plans, JCCs ensure youth and families complete Balanced Alliance forms, JCCs and CJMs attend FFPS consultations sessions that are principally conducted by the CJMs, CJMs coach and mentor JCCs, CJMs complete GRM quarterly (30-item form that records the model adherence and competence of JCCs in all three phases), CJMs observe each unit JCC's family sessions, and CJMs listen to digital recordings of each unit JCC's family sessions. 
This study addresses the following research questions:

1. What are the Juvenile Court Counselors', the Community Justice Managers', and the Senior Managers' opinions and experiences with the implementation of FFPS core components?

2. What are the Juvenile Court Counselors', the Community Justice Managers', and the Senior Manager's experiences of what individual and organizational factors that assist or impede the implementation of FFPS?

3. What are the Juvenile Court Counselors', the Community Justice Managers', and the Senior Managers' opinions on the advantages and disadvantages of FFPS as a case management model?

\section{Discussion of Themes}

Five themes emerged from the aggregation of the observations of the consultation sessions, the observation checklist, and the transcripts and written notes of the JCCs, the CJMs, and the Senior Managers. Depending upon the purposes of a qualitative evaluation, themes can emerge inductively (Charmaz, 2000; 2006) and deductively from the program literature (Patton, 2002). The themes that emerged included the core components of FFPS implementation and the individual and organizational-level factors that assist and preclude FFPS components from the observations and self-reports of the JCCs, CJMs, and Senior Managers. The themes also include the JCCs opinions and perceptions on the advantages and disadvantages of FFPS as a case management model 
and their recommendations on how to address remaining individual and organizationallevel challenges. These themes were as follows:

1. FFPS implementation is facilitated by JSD's culture and its resources.

2. FFPS advantages as a case management model.

3. FFPS disadvantages as a case management model.

4. FFPS implementation of core components is challenging.

5. FFPS requires organizational and individual-level changes

FFPS implementation is facilitated by JSD's culture and resources. This theme revealed that Multnomah County Juvenile Services Division is a culture rooted in rehabilitative approaches and is always the "first" among juvenile justice agencies to try new innovations. The interviews and observations answered one of the research areas, “What organizational and individual-level factors assist or preclude implementation?" In discussing culture and innovation, some JCCs mentioned that the educational level and training of the staff helps FFPS implementation. Moreover, the JCCs' offices have numerous IT resources, such as laptops, computers and cell phones to complete FFPS paperwork and schedule appointments with families while out in the field.

CJMs, JCCs, and Senior Managers indicated during the interviews and during Functional Family Probation Services consultation sessions that they believe in applying rehabilitative approaches toward the county's youth and families. CJMs and JCCs indicated that despite their simultaneous role as a social worker and court officer 
accountable to public safety, most of them foremost consider themselves social workers and have bachelors and graduate degrees in social work, counseling, criminal justice, psychology, and education. Some JCCs indicated that they were already providing similar services to FFPS, such as using motivational interviewing skills with families in the home. One JCC confirmed:

I mean we have always been in the home, we have always worked with families, so this is not uncommon for us, but for some other folks [other JCCs] it is uncommon. Not much of a change for us with FFP. I'm still using a lot of MI [motivational interviewing]. We got trained on MI [motivational interviewing] a couple years ago. Honestly, it was just about learning the verbiage.

A few CJMs, JCCs, and Senior Managers indicated that because of tight budgets and the need to reduce recidivism, JSD needed to look toward "new" and "different ways of doing business." Staff indicated that FFPS was thought to be suited for JSD. One CJM indicated that the JCCs and CJMs have the education, background, and experience giving them the ability to deliver FFPS and to understand and grasp its goals and principles. In other words, JCCs and CJMs were expected to easily learn and acquire the skills of FFPS, and FFPS would easily be incorporated into JSD's culture and structure. One JCC pointed out:

We have a very talented, intelligent group of people here who have educations in counseling, psychology and social work. Most of us even have master's degrees. 
We are here because we know locking kids up doesn't work, what works are practices rooted in treatment. The county does provide us with laptops, phones, offices, and cars to reach out to these families. We just have some more kinks to work out.

A CJM affirmed:

The fact that we do not have an agency culture that is correctional ... Well, if you [an agency] are very correctional, it [FFPS] will not work. Like I have one family that is from California and that state is very correctional. I just can't imagine how they are doing FFPS in LA with guns, and to my understanding it is mandatory. FFP is not forced on our families. If they don't want it, we won't force it, it doesn't happen though. Still, in Caly, they go into people's homes with guns. We don't do that, we're really social workers and this is what we do.

Other JCCs and CJMs indicated that Multnomah County is forward thinking and most of the time is the first to implement and make changes. One JCC joked:

We always have to be the first. I get tired of it sometimes. I mean people from all over come to see how we do things. We not too long ago had people from New Jersey looking at how we do things here.

A few JCCs and CJMs indicated that to make changes strong, supportive leadership is crucial. CJMs and JCCs commented that their leader believes in FFPS and has hosted meetings for all staff at all levels to discuss how to improve FFPS 
implementation. Further, JSD leadership, and for some, staff belief in the model helps JCCs and CJMs who want to make it work. One CJM stated:

Christina [the Assistant Director of JSD] believes in FFP and has been a cheerleader for us. There a lot of us who agree with her, but it will take time and resistance from certain people we did expect to happen. So unfortunately, not all of us are on board, but they're not on board because they are just burnt and have been doing this a long time. It's not that they don't believe in it [FFPS]; it's just that FFP is extremely time consuming and demanding.

One Senior Manager added:

We have been suffering budget cuts for a long time, but it is time for us to do more, everyone is doing more these days to show we are worth our paycheck or we'll be let go. It's not as bad as some people claim, yes, it is more work. But my worth is how I can reduce recidivism and increase public safety that is how I view it. Those, which are few of us, only want to still only do check-ins.

One JCC explained:

We've been experiencing budget cut backs for the last ten years here [at JSD] also especially out in the community and in services. This is the county's way to try to give the youth and family, hopefully, something. You can't reduce recidivism without giving them [youth and families] help.

All JSD staff indicated that FFPS requires agencies to have the technology to keep 
up with FFPS case plans and paperwork requirements. FFPS paperwork helps JCCs stay focused on program fidelity and the goals and themes that each family needs to work on. Prior to implementation, JSD had desk top computers, laptops, county cars, and cell phones for all its JCCs, CJMs, and Senior Managers. These technologies save time and keep paperwork up-to-date. A JCC added:

Because at times I can't get everyone together, we've used our cell phones to include all family members. The laptops took time, but using the laptop is the only way I can keep up with my work. We wouldn't do well without them. Ya can't get back to the office to do your work; you're on the road a lot.

FFPS advantages as a case management model. This theme answers the research question, "What are the advantages of FFPS as a case management model?" Most JCCs explained that most families appreciate the JCCs helping them and it gets the parents to parent and be part of the court process. Other advantages include the JCCs can better address the needs of the youth and family by entering the environment and home that the youth comes from and who will be returning after detention or placement.

JCCs indicated that many of these families live in extreme poverty and realize they need help. JCCs and CJMs indicated that working with families in the home allows them to better address the youth's delinquency. A JCC elaborated that families do appreciate the services: 
Many of these families live in such extreme poverty and circumstances, experience joblessness, drug \& alcohol abuse, and domestic violence. It's hard for them to get out from under. With FFP, well most families love it, they like us coming in and helping them out in the home. They also like how they don't have to come to juvenile. It saves them time and money for them on gas and transportation. This also helps me know what's going on in the home and I can help the youth better that way ... the youth is more compliant because the youth knows I'll be coming around.

One JCC stated that all families are different, but for the most part FFPS is effective and has observed improvement in family communication and functioning. Many JCCs indicated that they do see the family relationships improving.

I do see a difference; I see family communicating and understanding each other more. If we can get them communicating, the family is not so defensive. When they do get defensive I can break that so they can hear each other more, that's great. With one family they are always arguing, so with this [FFPS] is getting them see a different kind of communication.

Moreover, JCCs and CJMs stated that FFPS is effective for families struggling with mental health and substance abuse issues. One JCC illustrated:

Everyone benefits from FFP. Even with families with high mental health and A \& D issues, our people still benefit. I have a case where the dad is an alcoholic and I 
couldn't engage him before 10:00 a.m. Now I get there before 10:00 a.m. And he can see the connection he has with his daughter and how and what he does impacts her. I also have other families with serious DV and mental health problems. The other benefit of FFPS is that it ensures parental accountability and engages parents in the court process. Often JCCs emphasized that they feel like they "parent" their kids on community supervision. At the same time, the juvenile justice system in the past pulled the youth away from the family or did not include the parent's in the court process. From this investigator's observations, most parents assume that probation officers and the bench remove the youth from the home or take sole responsibility of a youth. One JCC thought:

The system in the past separates families, and sometimes they do need to be separated, for example the youth was abused or mom and dad are junkies. Yet, what caused the delinquency comes from the home and I can address what's going on the home to prevent future delinquency. If ya just pull the kid out of the home and not deal with the problems in it ... well, the kid is only going to eventually go back into the home anyway, well at least sometimes. This gets parents engaged in the youth and in their problems. This also makes parents accountable to the youth and gets them involved in the legal process. Too often we are only working with the kids and parents expect us to raise their kid. No, we get the parents to parent. 
However, a family that is struggling and that doesn't get along is tougher to work with. It takes a lot of work on the part of the JCC to get the family communicating. A JCC explained:

The families are using the FFP language, it is helping them and I do see a difference; however, families that are more chaotic, hate each other, are constantly in crisis they eventually fall apart with or without FFP, there was already years of damage, but we are at least supposed to try, and my CJM understands, and that's o.k.

Another JCC briefly gave a story of a family:

This family did eventually fall apart, mom, living with a boyfriend and her kid [on probation] was trying to quit the drugs, but bio-dad admitting to liking his drugs still and was not willing to give it up. When mom could not take care of her kid anymore, she just split and abandoned her kid. FFP was not going to work with this family, they just either didn't want to or just couldn't.

One JCC expressed concern that she was not doing the model correctly when her families were still falling apart:

Ya know, we'll never say it, even to each other in this building, but we all know, even those of us who been here a long time that when families quit and can't get it together ... I think there is a sense of blame [JCCs blame themselves]. But at least being in the home I can better address the needs of the youth and family and 
hopefully keep the kid from trouble and get the parent to parent.

A few families have not wanted the JCC in the home, but were still open to see the JCC. Instead a few JCCs are meeting entire families in public locations, such as in a fast food restaurant, Burgerville. Some JCCs indicated that families are embarrassed by their home conditions. JCCs are advised to still work with the families even if it is not in the home. One JCC said:

It is rare that families don't let us in the home. I'm o.k. with meeting them in a public place if they want. We are allowed to do that and I find that delivering FFP is just as effective. I think it is effective because we are supposed to match to the family. [matching is a FFPS principle]. FFP won't work if they don't want me in the home, it works best when I meet them on what they need and want. That's just commonsense.

The FFPS model requires that all the JCCs use its skills and techniques. However, despite FFPS having its own skills and techniques, JCCs indicated that the model is flexible. JCCs and CJMs have been trained that they are a required to meet families a recommended amount of times at each stage. However, depending upon the needs of the family, the model allows for more or fewer meetings. One JCC stated:

Yes we do have more paperwork and I'm on the road more, but the model [FFPS] like our [FFPS trainer] says and my CJM says, the model is flexible, I mean we've got a lot of flexibility during the phases and as long as you use the skills. I 
don't feel that I've lost my independence with this model. I am still using some of my own style I use along with FFP. I got more work, but I still got my flexibility and independence. Our CJMs know we need that.

FFPS disadvantages as a case management model. This theme answers the research question, "What are the disadvantages of FFPS as a case management model?" A few JCCs and CJMs have mentioned the disadvantages of delivering FFPS, such as lack of family responsivity, concern over safety issues out in the field, concern over increased workload, increasing burnout, and concern about working late nights. JCCs that were cynical and who deemed their responsibility primarily correctional expressed concerns that some families are so chaotic that FFPS is not enough and they expressed they are too busy to deliver a intervention that is too short to help families who suffer from years of multiple problems. JCCs added that it would be a waste of much needed time, and even so, not all families want to change or be responsive to the model or any model delivered by a JCC, a family therapist, or anyone. JCC poignantly stated, "I think it is really a neat model, family models we are told are supposed to work, well, good, then give these families to someone who is not as spread thin as me, they deserve more than just a PO to give them counseling." Other JCCs were concerned that some families are just not willing to change, but stated that management and CJMs are aware that FFPS is not for everyone. One Senior Manager stated, "We know it will not be for everyone, but we at least we got to try." 
One disadvantage is that the dual nature of JCCs as court officers and social workers delivering FFPS in the home can be conflicting. Many JCCs like the dual role that is encouraged at Multnomah County Juvenile Services Division. However, for some, there is conflict especially for those who work in the RISE, formerly the gang unit. A couple JCCs indicated that their primary role is enforcement of court mandates and public safety. One JCC complained:

Family counseling is a whole new ball game. They tell us it's not family counseling that it's a case management model ... If I have to go into the home and get people to talk. Well, THAT is family counseling. THAT takes a lot of time, which I don't have. My job is public safety. If my kid goes out and shoots someone or is on the news, I feel awful. If the county wants youth in family counseling, then the court needs to make it mandatory that kids and their moms and dads go [to family counseling] to an experienced family counselor.

Another JCC understood the conflict that the other JCCs suffered:

I like the model, I see improvement with my families, but for those who work in the gang unit, that gonna be harder for them because those kids and families are high-risk and high-needs. AND ya got safety to think about. Using FFP with those families, I don’t know, time will tell.

One JCC insisted: 
I understand why FFP was chosen. We understand that family-based interventions 'work.' We had to do something that was better than before. However, FFP appears to be a great model, but when you go into the home and deliver a model similar to therapy and gain the trust of the family, but when the family is so chaotic, and bad for the child and you have to pull the kid out of the home. Well, you just destroyed their trust. The JCCs are struggling with that. We are just told that the JCC failed because the kid gets pulled out of the home. Nonsense, the purpose of FFP is to make parents more accountable and for them to learn how to deal with their own problems. Still these families are the highest of the high, sometime no matter what we do, the family just can't get it together, with or without FFP.

JCCs knowledgeable of other JCCs who find that applying FFPS and then having to pull the youth out of the home could destroy the trust of the family suggested that it's possible to continually keep the trust of the youth and family if the JCC applies the balanced alliance technique and be upfront with the family initially. A JCC explained:

If you explain to the family up front your role and that you are there to help, and are up front with them that you are also there to increase public safety ... well, that is where you begin building trust.

A JCC commented that the model is meant to make youth and parents more accountable, but no matter what skills and techniques used, some JCCs think that the 
model makes the family more dependent on the JCC and not accountable and responsible to the youth or the Court. One JCC lamented:

It's a shame; the odds are piled so high against them. Still, I feel no matter what I do I am enabling poor parenting. By me going to their home, it means they don't get out of bed to get to JSD, I gotta do it. Also, the parents are dealing with their past abuse and their own criminal history. It is like an uphill battle. I think the kids are getting more out of it than the parents. Occasionally kids do embrace making positive choices, so the FFP model is helping them. The kids realize that they don't want to be like their family. I'm worried that no matter how much I improve on FFP, I'm still not making parents accountable for his or her youth's behavior. We are going into the homes, so parents can stay at home and not get out of bed. I've even noticed the other JCCs get discouraged when families still fall apart after doing months of FFP with them. We're exhausted and FFP doesn't work for all families, especially these really high need people.

Another JCC pointed out that not only had the workload increased but now the JCCs have to work with both the parents and the youth, which increased the workload and caseload more. Also, JCCs indicated that not all parents want to be included in the process. A JCC said: 
Now I have to deal with both kid and parent. Before I only had to work with the youth, even I said to the parents before 'they are not the ones on probation.' Now parents complain that they are not the ones on probation so why do they have to deal with it. What I learned was to get the parents involved as my second set of eyes. I mean it can be hard working with nasty parents, but it beats me searching for kids. It's still more work though.

Interviews and observations of FFPS consultation sessions revealed that using the skills sets and principals of FFPS, such as maintaining a balanced alliance and facilitating communication between the youth and parent, parental dependence on the JCCs will diminish. However, staff indicated that FFP is not for all families. Some JCCs during FFPS consultation sessions stated that the home environment is unhealthy and a youth keeps running away from home. A JCC complained:

I'm told that as long as I do what FFP says for me to do with these families they will get better, well, it doesn't work for as many as we were told it would work for. Same ole, same ole. I'm told I ain't doing it right, well, fine. I'll work with families where it does work.

Another JCC agreed:

The balanced alliance and getting them to work together and work out their stuff does help, but it's still hard to get these parents who struggle for so long and who have developed poor parenting skills, which is generational. To get them to 
change, and parent effectively ... well they learn from their parents how to parent poorly, it takes a long time, but I still don't think FFP is for all families.

This investigator noted during FFP consultation sessions and interviews with JCCs that family functioning and communication is improving, but some JCCs stated that anecdotally they are still doing the same amount of probation violations. One JCC stated: My families do appear to be functioning better. Still, I think I am doing the same amount of probation violations. I'm not sure. R \& E will figure that out for us. Well, they're not getting worse. The ones that do really well, well, you just don't see them or they don't need you as much anymore. That is the purpose of FFP is to get them to solve their own problems, make parents accountable to their kids, and not rely so much on the JCC.

One JCC expressed concerns about non-FFP youth:

I have both FFP and Non-FFP youth. My non-FFP kids are getting "shortchanged." I'm seeing families whose kids are still in their homes, but kids who are in placement or in a proctor or DHS homes I'm having a harder time getting to them. They need help too. We've been depending on our interns to see our kids [who are not with their families].

Another JCC expressed that they have less time to get to know the youth individually and find out what's really going on with the kids.

One JCC lamented: 
I used to have the kid come in and talk. I'd find out what was going on with him, especially working with gang youth, ya need information, intel on what's going on, now that I go in the home it's hard to bring up what's going on with the kid in front of the parents, the kids just clams up. . . Also, for boys they're not gonna talk about boy things in front of their moms. No way. I still gotta see these kids without mom, especially an over-bearing mom.

Another JCC said:

I have this obnoxious parent that won't let the kid talk. FFP is to get everyone to talk to each other, but she won't shut up, and I don't think the family is truthful on what is going on. When I get the kid alone in my office I can find out what the kid is struggling with and what their problems are. Me going into the home with this family is not helping this kid. I'm supposed to do the balanced alliance, but this woman is VERY obnoxious. FFP won't do.

Another JCC commented:

I know right now we're trying it out and all families are different, but really, it's not really that effective for the really, really high risk families we've got. The parents just continue poor parenting and enable their kids. There are continual uses of drugs and alcohol among the parents. Parents are dealing with their own past abuse and some their own criminal history. It's an uphill battle. It's not beneficial across the board. We need to better assess who will be good for FFPS 
and who won't. I want to help youth, but this is NOT making parents accountable like people want, We go in and parents think it is great, 'I don't have to parent my kid.

From the interviews and observations sessions, JCCs indicated that few families, but not most families on their caseload are not responsive. During FFPS consultation sessions, the CJM and other JCCs brainstorm what other skills and techniques the JCC can employ. CJMs encourage the JCC to try again and other JCCs offer emotional support. The purpose of these sessions is for the CJMs and other JCCs to offer feedback and coaching so as to improve model competence of the unit. What also has been noted by this investigator during these sessions is that these sessions give JCCs time to vent, relax, and seek support from other JCCs delivering FFPS in the unit. Yet, JCCs express that their families are still struggling.

Another issue with FFPS is that it requires JCCs to visit the youth and families in their homes. Sometimes these homes are in areas where a JCC may perceive as unsafe. Several JCCs and a few CJMs expressed that for some FFPS raises safety concerns. JCCs explained that several of them are women, who have limited knowledge of the use of force, and because of FFPS they are working in the night late. JCCs and CJMs stated that self-defense training should be mandatory. JCCs suggested that JCCs should go to family homes in teams, but said because of workload they would not be able to visit homes in teams frequently. A two-day self-defense training was mandatory during the first year of 
implementation. This investigator attended the two-day trainings. One JCC expressed concern:

We go into homes and neighborhoods that are unsafe; we are too busy to go out as a team. Because we go into the homes we should have similar adult side safety protocols.

Several JCCs indicated that safety protocols that are required in the adult probation side should be mandatory across all units at JSD. One JCC indicated:

My CJM does not make me go into homes that are unsafe and my CJM goes with me if I feel unsafe. However, this should be mandatory across all the units, other CJMs don't take safety seriously and are jeopardizing the safety of their JCCs by denying that this job is not dangerous.

Those who work primarily with gang-affiliated youth expressed the highest concerns.

One JCC stated:

Of course we have to worry about safety. For example, I got a youth's mother that was shot at. I have a whole family involved in a gang and they have had their homes attacked. When I'm in the home doing FFP I'm in my vest. I am sitting on the couch facing the door and looking for exits. Our unit only got vests two years ago and we don't have many of them. Now that this unit is growing we need more vests and more protection. 
Other CJMs and JCCs argued that going into the home is part of the job and that none of the clients have ever harmed a JCC. It is part of the job and they know the risks.

One JCC reasoned:

It's part of the job, we go into the homes, we take risks, I'm not afraid; I just don't think about it. There is more of a likelihood of getting mugged out in the street than being harmed by one of my kids and families. They are not going to hurt me. They WILL go to the Oregon Correctional Facility if they harm any of us. Many JCCs with smaller caseload sizes have managed to deliver FFPS with the time and dosage that is required. However, JCCs with larger caseload sizes, especially in the RISE unit have stated that it is difficult for them to provide the dosage and the time required for each phase. Moreover, because of court responsibilities and scheduling JCCs state that the only time to get all family members together especially when youth are in school is at night. One JCC stated:

We don't do FFP as often as we can and we are working into late nights, for that is the only time you can get all family members together. Kids don't come home from school until after 2 or 3 during the school year. During the summertime it is easier. Still, with my workload, travel time, court hearings, court paperwork, FFP paperwork, and school visits, there is just not enough time in the day to do FFPS the way FFP requires. I maybe at most I can do 1 to 2 FFP a day.

Another JCC indicated: 
Biggest issues is time with FFP. The paperwork and you're on the road a lot. It's crazy, got to get the car, got to check it out, get gas, and then some days you're going to Troutdale, some days going to Northeast then Southeast. I think that is probably where people feel so stressed. In the old days we had centralized caseloads, where one would cover the northeast, or in just in one area. Now we are spread out everywhere.

One JCC suggested:

I'm really organized, yes, and I get to use the skills. There are some units where their caseload sizes are too big, but they also need to get more organized. I got some weeks I'm running around like a crazy, and then other weeks I got a handle on it. Just depends on organizational skills.

Another issue is that at times, it is difficult to get all family members together at the same time. FFPS requires that all family members be together during FFPS sessions. One JCC stated:

You know it is still hit or miss, sometimes its only one mom or one dad, and more or less the siblings have not been invited, sometimes a sibling will still sit in. As long as you have a kid and a parent. We can count it. No matter what or who is at a session, I use the FFPS techniques.

FFPS implementation of core components is challenging. This theme answers the research question, "What are the JCCs and CJMs experiences with implementing 
FFPS core components?" For organization and reading ease, this theme was divided into sub themes that provide data on each FFPS component. The sub themes include: JCC self-reports on competency, CJM coaching and monitoring during consultation sessions, FFPS case plans and paperwork, Global Rating Measure (GRM), and Balance Alliance Questionnaire (completed by youth and parents). As written in Chapter One, JCCs and CJMs learned FFPS skill sets and techniques during training and the JCCs deliver the model the recommended required amount of time and dosage. The JCCs have the youth and family complete the Balance Alliance form after the first 30 days of FFPS services. CJMs provide coaching at consultation sessions, rate JCCs quarterly on the GRM, and CJMs are required to observe and listen to recorded family sessions.

\section{JCC Self-reports on Competency}

One of the limitations to this study is that this investigator does not have experience in FFPS to observe and rate the JCCs' delivery of FFPS during family sessions. The literature recommends that experienced FFT/FFPS counselors can rate the competency of counselors delivering FFT/FFPS during consultation sessions (Sexton, 2011). This theme uses the self-reports of the JCCs on their perceived competency. Other JCCs indicated they forget the stages and have to refer back to the manual sometimes. One JCC stated that working in teams helps:

I sometimes work with another JCC and while we drive to the home we talk together about the family's theme and we discuss what FFP techniques we'll use. I 
mean, using 'noble intent' and 'reframing' is easy, just other stuff can be hard on knowing when to use it. I sometimes use must MI, MI gets them to talk.

JCCs that still struggle with learning the model blame the infrequent consultation sessions and the resistance from others who do not attend the sessions or contribute to the sessions. A JCC stated: I can't get it when we're all not there to talk about it so we can get coaching and train each other, these FFP meetings are just turning into case staff meetings ... if we need to learn FFP why don't we just combine FFP and case staff meetings. I feel like they are a waste of time." Few JCCs indicated FFPS is just motivational interviewing and state the CJMs are running their FFPS consultation sessions differently and because of that the JCCs are learning it differently. One JCC opined, "It's just motivational interviewing, not a big deal, and the 4 CJMs do it different. I am still required to go and I go."

This investigator observed that two of the units out of the four have meetings weekly and are attended by most unit JCCs and the CJM. This investigator prior to this study, attended FFPS trainings, FFPS consultations in the first year of implementation, and delivered FFPS with fellow JCCs. Despite this investigator still learning FFPS, other JCCs indicated and this investigator noted that when CJMs are present they do have different approaches to running their FFPS consultation sessions, yet their coaching is helpful and effective. A CJM was aware that JCCs consider FFPS as only motivational interviewing and stated, "FFP is not MI, MI is a great technique and can be used, but FFP 
has phases, matching and balanced alliance is one of the essences of FFP."

When this investigator probed further on the JCCs perceptions on how they were

performing using the "balanced alliance" principle statements were mixed. As defined in Chapter One, the balanced alliance principle requires the JCC to not take sides with the youth or parent. One JCC stated:

I think I'm alright, but when the kid is screwing up and bossing the parent around, or the parent is verbally abusive to the youth, it can be hard, but it can be done. I talk to the other JCCs or my CJM on how to do it better. Our ratings that we saw last year showed we were doing pretty good, but a lot of us are still working on the balanced alliance.

\section{CJM Coaching and Monitoring during Consultation Sessions}

Within this theme the JCCs and the CJMs indicated that the JCCs and CJMs are progressing and learning the model, but a huge barrier was the lack of the ability for all JCCs to attend the FFPS consultation sessions. Moreover, this investigator noted from observing FFPS consultation sessions CJM differences in mentoring and coaching FFPS skills and principles. Further lack of attendance of JCCs in some units occurred and other units were not having the sessions at all. However, as noted earlier, when CJMs were present, CJMs demonstrated they grasped the skills and techniques and were effective. One CJM said: 
Yes, my staff is progressing. At first it took time to get used to some of the techniques. I even had to tell the JCCs not to spend so much time with families, telling them exactly what they should be doing in the home to save time. Though, I have noticed that in other units some still need to hone their skills in reframing, pointing, sequencing. Reframing can be difficult because if the family is fighting, it's hard to do a correct reframe to get them to stop fighting. Others just don't seem to be getting it. We did know that this would happen.

\section{A JCC added:}

I feel like I got a handle on it, but FFP takes time to learn. Most of us got it, they're just a few that are struggling, we all gotta help them get here.

This investigator asked how the JCCs could improve their competence in delivering FFPS.

One JCC suggested:

FFPS is hard work and everybody is learning it at different speeds, but that's because we need our CJMs to be consistent on providing coaching and feedback. People need to go to the FFPS consultations sessions. I know some CJMs are not holding weekly sessions. People are getting pulled to do other duties and not going to the sessions. Ya can't learn it unless if you don't get feedback and talk about your cases. I even need to go back into the manual to check what I am supposed to be doing. The CJMs need their workload reduced too. 
For some JCCs the change had been difficult, for many JCCs have grown accustomed to applying their own techniques. Further, this investigator noted from observations that CJMs and JCCs are struggling to find the time to attend weekly FFPS consultation sessions, and sometimes FFPS consultation sessions are canceled because of lack of attendance or the CJM cannot attend. CJMs indicated they struggle with finding the time to coach and mentor JCCs as well. Sometimes units held meetings without the CJM present and JCCs that were regarded by the unit as the unit JCC superior at delivering FFPS would run the meetings. Some JCCs indicated that the sessions are important to improve upon the model; others indicate they are too busy and that the sessions are not helping because CJMs who are required to provide mentorship and coaching are still learning it themselves. As one JCC said:

FFPS is hard work and everybody is learning it at different speeds, but that's because we need our CJMs to be consistent on providing coaching and feedback. People need to go to the FFPS consultations sessions. I know some CJMs are not holding weekly sessions. People are getting pulled to do other duties and not going to the sessions. Ya can't learn it unless if you don't get feedback and talk about your cases. I even need to go back into the manual to check what I am supposed to be doing. The CJMs need their workload reduced too. 
CJMs suggest that their workload be diminished to focus more on FFPS mentoring and monitoring. To date, JCCs highly skilled at FFPS are assisting CJMs coach and mentor other JCCs. One CJM said:

I am not perfect I am doing the best I can to give JCCs feedback and coaching. They're getting it. I get to see how they are doing. We're all getting there.

A CJM worried:

Besides providing coaching and feedback during the consultation sessions, I have to listen to the tapes of JCCs during family sessions, I have to observe family sessions, and keep up with the JCCs paperwork, I just don't have that kind of time. I've got too many other duties that are non-FFPS. The County should cut out some CJM responsibilities and make FFPS a priority so I can get the JCCs delivering the model consistently.

\section{FFPS Case Plans and Paperwork}

The purpose of the FFPS family service plans and paperwork are for the JCCs to track and document their model adherence. JCCs during the engagement and motivation phase work on the family service plan. JCCs are to meet the family within the first 10 days of the engagement and motivation phase two to three times. The family service plan is to keep the JCCs focused on a family's themes and goals.

A JCC said: 
You go to the house you have one to two meetings in the engagement and motivation stage and work on the service plan. This keeps the family motivated and so they understand that meeting the goals of the plan will reduce conflict and chaos. I treat it like a contract with my families. From what I hear others [JCCs] use it differently.

JCCs stated that there is too much FFPS paperwork that does not help them. One JCC stated that they do not have time because of other duties to complete the FFPS paperwork in a timely manner.

One JCC indicated:

Now I gotta do both FFPS paperwork and case notes in JJIS. Both don't help me. Just fill it out, when I get around to it or when my boss [CJM] tells us she'll be looking for them. I get behind. It helps you adhere to the model, and what to do for each session, but I just fill it out. It doesn't tell me what to do next. The consultation sessions help me figure that out. My CJM is a great coach and gives me feedback, that's what we really need.

Another JCC stated that the paperwork does have them adhere to the model better and stick to the goals and themes of what the family needs.

A JCC said:

FFPS family plans help me stick to the goals of the family at hand. It reminds me of the themes and goals I need to work on for each session. Once ya get your 
time skills down, it doesn't take long to do while on the road or in a coffee shop. For me my plans help me stick to the model. Can't remember all the tools I have. But, no I can't keep up with it, I'm constantly behind.

Global Rating Measure (GRM)

The GRM is filled out by the CJM to keep track and ensure JCC model adherence and competence. JCCs expressed concerns that CJMs are not skilled enough and are not accurate in how they rate each JCC. One JCC said:

My unit is fine, but we JCCs in all units have noticed that the CJMs are not providing consistent feedback and coaching. Well, they're still learning it themselves. That's another thing, the Global Rating Measure. Only some of the other unit JCCs know how they are being rated on the GRM by their CJM. I don't even know how I am rated. It bothers some us being rated on how we are doing when the CJMs are still learning it.

Another JCC indicated that they do know how they rated, "Oh, I know my rating, my CJM tells me. Because of the GRM I know that I am improving."

The CJM's were asked what they thought about the GRM. One CJM stated: The GRM is a great way to keep track, monitor, and keep a record of the feedback that we give to the JCCs. It's an excellent tool. The JCCs should not worry, the GRM is meant to be used as a learning tool. The CJMs have had training on how 
to become more consistent when we rate our unit JCCs to be fair. We're getting there.

\section{Balance Alliance Questionnaire}

Another form that is used to monitor JCC model adherence and competence is the Balance Alliance Questionnaire. Two Balance Alliance Questionnaires are given, one to the youth and the other to the parents to complete after the JCC begins FFPS services after the first 30 days. One JCC indicated, "the parents and the family don't get it, they don't understand why they are rating us.” Another JCC complained:

It's very awkward to have them fill this form out while they are in front of you [me, the JCC]. I wish we could just mail them out or have someone like interns just call the families. Well, I know if we mailed them out, we'd probably never get them back. Also 30 days is too soon, it takes quite a few weeks to build trust and rapport with a family.

Another JCC, who is bilingual, said that we need them written in Spanish for parents who can read Spanish.

Sometimes I have to read the questionnaire to them because they cannot speak and read in English. Well, not all of them can read and write in Spanish either. Still I have to interpret for them. It may take an hour for me to explain the questionnaire to Hispanic parents, it's really uncomfortable. Of course they are 
going to say I am doing a great job because I'm sitting there interpreting right in front of them.

Another JCC said and made a recommendation:

It's weird when English speakers [English speaking parents on caseload] don't even understand the point of it. Sometimes, they don't understand a question and I have to explain it to them ... I guess this is the only way we can get real feedback from the families. We need someone to interview the families and see how the families feel.

Another JCC stated what his CJM told him:

My CJM tells me that the balance alliance questionnaire form is helpful and the county needs them, but I haven't seen my results yet. I think some JCCs just stopped giving it to the youth and families. They just forgot about it. I'd like to get feedback on how my families are rating me. What's the point of asking clients to rate me and then I don't know how I am rated. Last time at a meeting, we did see the balance alliance results, but they put all the JCCs together. I want to know mine.

The CJMs were provided more training in September 2012 on how to better complete the GRM to increase CJM inter-rater reliability. CJMs attended one day training on how to complete the GRM and how to coach and mentor more effectively. To date, JCCs highly skilled at FFPS are assisting CJMs coach and mentor other JCCs during FFPS consultation sessions. 
FFPS requires organizational and individual-level changes and challenges. This theme answers the research question, "What organizational and individual-level factors are assisting or precluding FFPS implementation?" This theme also includes additional recommendations from JSD staff and provides the changes that JSD made during implementation to increase FFPS fidelity. JCCs, CJMs, and Senior Managers as said in the first theme, FFPS implementation is facilitated by JSD's culture and its resources, despite some of the resistance. Resistance was not a result of lack of belief in the model, but that a model based on therapeutic skills and principles should be delivered by family therapists and counselors, not delivered by those responsible for public safety. Nor delivered by JCCs who already have a lot of work they have to do. A few found no need for the model. One JCC stated:

This model cost a lot of taxpayer money for us to be trained and to pay FFT, Inc. I don't know why they did this, juvenile crime was going down anyway. Really, now I just don't have to only deal with the youth, but know I gotta deal with the whole family, it is more work that is not necessary.

Observations and the collective interviews from most JCCs did not reveal role conflict. Many of them like the dual role that is afforded to them. For example, they have the option and the ability to counsel youth and families and teach parenting skills if they have the time and if they deem the youth and family would benefit. Many found that 
FFPS was another tool they could use as part of their dual role, but some found FFPS to be too time consuming and not enough for what the families really needed.

Another JCC said:

Before we were all referring treatment services to youth, but some JCCs had kids come to JSD, other JCCs like going in the home. We all did different things. Some say we all were doing the same thing. I don't think so. Hmmm . . now we all gotta do the same thing and show it.

One JCC indicated:

I mean we, our unit, have always been in the home [FSU unit], we have always worked with families, so this is not uncommon for us, but for some other folks, in other units, such as the RISE unit, it is uncommon. The RISE unit deals with kids who live violently, it is different for them [the JCCs].

Another JCC stated that there was some resistance to the change at first, but later more staff became convinced that FFPS was a more effective approach, but there would always be those who won't do it. A JCC explained:

I signed up for the job to help kids and families make changes. If you want to work with kids and see changes; you got to work also with where they come from. CJMs and other JCCs just got to make the other JCCs realize that working with the families is how to do it. As said earlier, there was resistance from some JSD staff and these resistant staff 
demonstrated explicit and implicit resistance during the FFPS consultation sessions and during the JCC and CJM interviews. During the FFPS consultation sessions, JCCs that were resistant frequently forgot the FFPS phases and skill sets, or kept giving the CJMs excuses on why FFPS would not work with a family. Although it could be possible that based on the JCCs years of expertise, FFPS was not suited for the family. However, the frequency of excuses made it difficult for an observer to determine if in fact FFPS was not suitable for a family, or the JCC was not using the model's suggested approaches and skill sets. Other forms of resistance were revealed when JCCs did not participate during the session's discussions. CJMs tried to convince the JCCs to try harder with families and offered new ideas on helping the families.

The JCC and CJM interviews also revealed a poor climate among some in the form of burnout and cynicism. Some JCCs kept forgetting to complete FFPS paperwork, did not take the paperwork seriously, forgot the phases, or the JCCs were picking the “easy" families to work with. Others indicated that FFPS was not as effective for the level of workload especially those with heavy caseloads. One JCC indicated, "we were convinced to choose this model, but we were never told about the level of paperwork and how much time this model would take. It seems that there was an expectation that this model can do more then what it does." Another JCC lamented, "this model and me doing it is not enough, and these families are not going to change, they have been having problems for so many years that it is ingrained. Only doing this for a couple of months is 
not enough.” Another JCC stated, "I am tired of running around after these kids, now I gotta do FFP, no way. Plus, are caseload sizes will always creep up.”

Overall a poor climate of resistance, burnout and cynicism from staff was not throughout the whole entire juvenile services division offices, for those who demonstrated and verbalized cynicism had long tenure working at Multnomah County Juvenile Services Division. However, other JCCs and CJMs with long tenure did not demonstrate cynicism, resistance, or burnout.

Another predominant issue was caseload size. Many JCCs with smaller caseload sizes have managed to deliver FFPS with the time and dosage that is required. JCCs with larger caseload sizes have stated that it is difficult for them to provide the dosage and the time required for each phase. It is common for a JCC to have a caseload size of 20 to 25 youth total, particularly in the RISE and Family Services Unit.

A JCC commented:

I'm at 13 [FFPS families], and I have 5 more that are not FFPS families, which is the lowest I have ever been, yet I am running around like a chicken with my head cut off. I know people were talking about us having 25 FFPS families or mixing both regular and FFPS families up to 25 . How am I going to do 25 when I can barely deal with under 15 . There is no way, and our supervisors know it. Fifteen to 20 is reasonable for a highly organized JCC. 
One JCC agreed that caseload size is an issue, but stated that it just takes getting better organized.

I'm really organized, yes, and I get to use the skills. There are some units where their caseload sizes are too big, but they also need to get more organized. I got some weeks I'm running around like a crazy, and then other weeks I got a handle on it. Just depends on organizational skills.

Another JCC said:

Caseload size should be no more than 15 to 20, especially with my kids [gang]. People are getting burnt out. A JCC works with a family for a month and a half, the family is still a mess and you're exhausted, and ya got more FFP families to deal with, so JCCs get discouraged. I think going in the field together to talk about the cases while on the road will reduce burn out and increase safety.

Another recommendation was that technology should be kept up-to-date and all FFPS paperwork should be integrated into JJIS and online to save time. Some said the laptops are still too slow and that desktop computers need to be upgraded. One CJM stated:

Because of FFPS paperwork, it should all be integrated into the JSD computer system for easy access. Still, our computers are slow and JCCs say that the laptops are dropping out on them while out in the field. We've been discussing what we need to do next to make FFPS paperwork and case notes accessible. 
Another CJM indicated that FFPS requires more reliability on technology that JCCs need to get used to. A CJM commented:

The purpose of providing laptops was so JCCs while on the road, which they are now on the road a lot, can work on their FFPS paperwork and case notes, at a coffee shop in between FFPS meetings, school meetings, court hearings, and treatment meetings. Management knows FFPS is very time consuming so we have provided technology to save time, the only problem is the old-timers have to get used to it. They complain that they don't have enough time. Really? If they use the technology right, they'd have more time.

The implementation of FFPS also required JSD to make changes in the Juvenile Justice Information System (JJIS) to better suit FFPS. One CJM reported:

For us to keep our case notes and FFPS case plans updated, we are talking about making adjustments in JJIS, so it tells me which cases are FFPS and which are not. We also integrated FFPS case plans into JJIS for quicker access.

JCCs and CJMs provided recommendations on how to improve FFPS fidelity. One CJM's quote captures what most other CJMs and JCCs stated:

We just need to prioritize FFPS a little more and make a couple more changes to so we reach 100 percent program fidelity. We need to keep caseload sizes down, negotiate with the union so our schedules are more flexible to the families schedule and the court schedule. We also need better computers, and get our CJMs 
on the same page.

Another recommendation was to combine FFPS consultation meetings and case staff meetings. The rationale for combining the meetings was to further reinforce FFPS knowledge and adherence and to reduce the number of meetings per week. One CJM stated, "I am in so many meetings, put me in less meetings and I can get more work done and help these JCCs."

After the completion of this summer study, in December 2012, JCC adjudicators, were trained on how to complete FFPS service/case plans. The intention was for the adjudicators to help the JCCs with paperwork and to introduce the youth and families to FFPS during the adjudication process prior to the youth and family case being transferred to the field JCC (email via Multnomah County Department of Community Justice listserve in December 2012). In addition, JCCs were provided the option to work eight hours, five days a week or work ten hours, four days a week (anonymous personal communication, December 2012). Most JCCs stated that working four ten-hour shifts was more helpful given FFPS's workload and the need to sometimes meet families in the early to late evenings. 


\section{CHAPTER FIVE}

Looking for alternative ways to reduce costs policymakers in criminal justice agencies have begun investigating ways to implement evidence-based practices. A review of the criminal justice and human service's literature provides guidelines and studies that discuss the individual and organizational features critical for the successful implementation of programs (Aarons \& Sawitzky, 2006; Fixsen et al., 2009; Farrell, Young, \& Taxman, 2011; Gendreau, Goggin, \& Smith, 1999, 2001; Joplin et al., 2004; Mihalic et al., 2004; White, 2006). However, Latessa (2004) warns that an agency that lacks effective quality controls and implements programs poorly will not have the ability to maintain program fidelity of an intervention. A qualitative process evaluation is helpful in determining which changes to to be made to ensure a model's program fidelity during implementation (Welsh, 2006).

This was a qualitative case study during the second year of FFPS's implementation at Multnomah County Juvenile Services Division. The purpose of this study was threefold. First, this project recorded the opinions and experiences of the JCCs delivering and implementing FFPS core components. Second, this project recorded the JCCs and CJMs opinions on the pros and cons of FFPS as a case management model. Third, this project recorded the JCCs, CJMs, and Senior Managers self-reports on the individual and organizational-level factors that assist and preclude FFPS implementation. 
Only two studies have examined juvenile community corrections professionals delivering and implementing FFPS and FFT. Rowland's (2007) study examined the model adherence and competence of juvenile parole officers delivering FFPS in the state of Washington. Rowland's (2007) study suggested that model adherence and competence is a moderator of paroled youth criminal behavioral outcomes. The second study by Gordon \& Arbuthnot (1988) examined the training and implementation of FFT among juvenile probation officers in Atlanta, Georgia. The study's primary results suggested that lack of buy-in from the chief probation officers and the additional workload brought on by FFT lead to FFT's elimination.

The intent of this study was to fill this gap and add to the body of literature a study recording the juvenile probation officers experiences implementing FFPS and FFPS's advantages and disadvantages as a case management model, and examine the organizational and individual-level factors that assist and preclude FFPS implementation.

This study addressed the following research questions:

1. What are the Juvenile Court Counselors', Community Justice Managers', and Senior Managers' opinions and experiences with the implementation of FFPS?

2. What are the Juvenile Court Counselors', the Community Justice Managers', and the Senior Manager's experiences of what individual and organizational factors that assist or impede the implementation of FFPS core components? 
3. What are the Juvenile Court Counselors', Community Justice Managers', and Senior Managers' opinions on the advantages and disadvantages of FFPS as a case management model? 


\section{Discussion}

The CJMs', the JCCs', and the Senior Managers' self-reports illuminated the individual and organizational features that facilitated and impeded FFPS program fidelity, as well as revealed their opinions on the pros and cons of FFPS as a case management model. The following discussion is an overall review and analysis of the results from Chapter Four. The following themes that emerged as the results of this study included:

1. FFPS implementation is facilitated by JSD's culture and its resources.

2. FFPS advantages as a case management model.

3. FFPS disadvantages as a case management model.

4. FFPS implementation of core components is challenging.

5. FFPS requires organizational and individual-level changes and changes JSD staff indicated that they believe in applying rehabilitative approaches to youth and families and understood that family-based interventions are evidence-based practices. Most staff said that Multnomah County JSD as a Juvenile Detention Alternative Initiative Site (JDAI) was highly innovative and supportive of trying "new" interventions based on evidence-based practices. Most JSD staff suggested that the workload required of FFPS should receive greater priority indicated to this investigator that FFPS's adoption fit JSD's philosophy and mission. 
Over half of the JCCs and CJMs were comfortable with the dual role afforded to them in their profession and indicated that FFPS increases that dual role. However, some JCCs and CJMs who struggled with FFPS found the dual role conflicting because they deemed their primary responsibility was toward public safety or they believed that delivering a family-based intervention was not the role of a community corrections professional. For example, a couple JCCs and CJMs lamented that a counselor role is based on building trust with the youth and family and this is difficult because a JCC might have to remove a youth from the home. JCCs indicated that this may break the trust of the family and threaten the counselor/client relationship. It could be possible that because of this conflict they may lack of belief in the model, or they may never have became skilled in applying FFPS. This investigator noticed that the JCCs and CJMs who indicated this conflict rarely held FFPS family sessions, rarely attended FFPS consultation sessions, or they frequently forgot the phases and skill sets of the intervention.

Some of these JCCs indicated that FFPS is not for all youth and family and they did not show initiative with families they deemed not suited for the intervention. However, it should be noted that some JCCs and CJMs during the first year of implementation stated during their interviews that they were not convinced of the intervention but later became more convinced of FFPS's efficacy going into the second year of implementation. These JCCs and CJMs may be the ones who mastered delivering 
FFPS to families or have families on their caseload who would be responsive to any intervention.

As for the remaining resistant, it could be possible that they did not want to believe in the intervention because of the increased workload required of FFPS or they may be burnout and cynical, which can occur among long tenured community corrections professionals.

JSD's IT resources such as cell phones, blackberries, laptops and desktop computers were already available to JSD staff. After implementation JSD staff realized IT resources besides model adherence and competence were critical for program fidelity. Prior to implementation CJMs and JCCs who chose FFPS were not aware of the high amount of paperwork required of FFPS. JCCs, CJMs, and Senior Managers realized after implementation that the paperwork needed to be integrated into the JJIS system and it needed to be readily available via the internet or among shared folders in office desktop computers. Because of the high level of bureaucracy at the Department of Community Justice, integrating the FFPS paperwork into JSD's already existing IT resources took months. What further exacerbated this problem was that some JCCs were not accustomed to using JSD's IT resources effectively and efficiently and most of them fell behind substantially in their FFPS paperwork. Moreover, JCCs indicated that while they were out in the field some of their laptops were "dropping" off the internet, keeping them from doing their paperwork while on the road. 
The following discusses the advantages and disadvantages of FFPS, as well as the individual and organizational challenges during FFPS implementation. The self-reports of the JCCs and CJMs indicated that a JCC's access into the home allows them to understand the problems and issues in the home. It also gives the JCC an opportunity to help facilitate and increase family communication while inadvertently teaching parents parenting skills. Frequently, JCCs indicated that the youth does not always disclose the problems in the home and this makes it difficult for the JCC to refer the youth to appropriate services or counsel the youth on predominate issues in the home. Further, some JCCs did notice some families communicating better. It could be possible that those who perceive increased family functioning were skilled in FFPS delivery or the youth and family showed better behavior when a JCC is in their home.

The JCCs and CJMs opinions on the disadvantages of FFPS included many factors: increased workload, working late nights, working with families who are not willing to change, safety issues, and the lack of time to work with youth individually. JCCs complained that they did not have enough time in the day to deliver FFPS with the required dosage and frequency along with their own court duties. Some CJMs indicated that they did not have the time to hold frequent FFPS consultation sessions, nor compel JCCs to attend the FFPS consultation sessions when a JCC was required to attend a court hearing. Sometimes FFPS consultation sessions held by the CJMs devolved into a case staffing session. This may be because of the CJMs and JCCs increased workload, but also 
because some JCCs and CJMs were not skilled at conducting FFPS sessions or not bought into the importance and need of the FFPS consultation sessions. On the other hand, other CJMs held frequent FFPS consultation sessions and demonstrated skill in providing coaching and feedback to their unit JCCs. Overall, those who were aware of the need of the FFPS consultation sessions and were "bought" into the FFPS model held and attended FFPS consultation sessions.

The other disadvantage reported by the JCCs was that FFPS is not for all families and their FFPS workload and caseload size was keeping them from working with nonFFPS youth. The JCCs expressed concern that families that are too chaotic and don't want to change are not suitable for FFPS and that they needed to work with the youth individually. It could be possible that these JCCS have not mastered the skills of FFPS to convince the families to change, or maybe the families don't need to change. Observations of family sessions would have helped with understanding this phenomena and what the JCC means by needing the family to change. The goals of FFPS is to reduce costs by keeping the youth in the home, increase problem solving, increase family functioning and communication (many forms of family functioning), and decrease youth recidivism. Another concern indicated by some JCCs was that they had little time to work with their non-FFPS youth. Non-FFPS youth were youth who were in foster homes or in proctor homes and not in the care of their immediate families.

Besides the additional workload, a few JCCs and CJMs indicated concerns over 
safety. They mentioned that because they were mostly women traveling into unsafe neighborhoods, particularly in the evenings that CJMs should encourage them to work in teams. Some suggested that they should have safety protocols similar to probation officers on the adult side. Some JCCs indicated that they will not have family sessions if they fear the area or the family. Most JCCs are unable to work in teams and because the JCCs are not sworn probation officers they do not carry sidearms and are not proficient in self-defense. This issue may affect FFPS dosage and frequency which JSD may need to address.

The other remaining individual and organizational-level "growing pains" included some resistance, cynicism, and burnout, as well as the CJM's inability to monitor the JCCs FFPS model adherence and competence and infrequent consultation sessions. Some CJMs said they did not have time to hold FFPS consultation sessions and expressed concerns about finding the time to observe or listen taped FFPS family sessions. It could be possible that more coaching and monitoring from CJMs could help reduce JCC resistance and help the JCCs who were still struggling learn the model. CJMs are also required to complete the GRM, which is a rating of the JCCs model adherence and competency. The JCCs expressed concerns over their personal GRM ratings because CJMs who were not observing family sessions or holding frequent FFPS consultation sessions would not be familiar with the JCC's competency yet they were still rating the JCCs. Further, the fact that the all FFPS paperwork was not fully integrated into the JJIS 
system or the JJIS system was not set up to track the JCCs dosage and frequency made it difficult to enforce the dosage and frequency required of FFPS. One Senior Manager had an intern manually track the dosage and frequency of the JCCs FFPS delivery; however, the work was time-consuming.

\section{Policy Implications}

JSD leadership has made efforts to increase FFPS program fidelity; however, there are still remaining individual and organizational-level barriers that need to be addressed to increase FFPS program fidelity. Based on the results and the previous discussion, the following are recommendations that may help increase model integrity.

FFPS consultation sessions should be mandatory and held weekly. To reduce resistance, encourage emotional support, increase monitoring of the JCCs, and increase JCC competency, everyone must attend these sessions. Resistance may be reduced if JCCs hear some of the benefits of FFPS and realize that the agency will be taking FFPS implementation seriously. This investigator observed that those who did not have or attend FFPS consultation sessions did not take FFPS seriously and were "hoping the county would stop the implementation." Further, JCCs and CJMs together can provide coaching and feedback for those who have tough cases, as well as provide emotional support needed for those who are cynical and burnout. These sessions may also help those who are struggling with the increased workload and dual role required of FFPS. If the CJMs and JCCs do not see the cost/benefit of FFPS there may be continual resistance 
and those who support the model may get discouraged.

Integrate all FFPS paperwork into the shared computer drives and on laptops.

Allow the the JJIS system to be accessible via online. The JCCs can complete their paperwork while on the road in between family sessions and court hearings to save time.

Maintain caseload sizes below 20 youth. The JCCs workload increased. Keeping their caseload size below 20 will allow them to provide the required frequency and dosage of FFPS. The manual and the research suggest that the higher the model adherence and competence, the more likely juvenile recidivism will decrease and family functioning will increase (Barnoski, 2002, 2004, 2005). Further, JCCs who see improvements in the youth and family may be less resistant and may buy into the benefits of FFPS.

The JCCs' FFPS frequency of family sessions in each of the three phases must be tracked in the JJIS system. In addition, FFPS paperwork for each session should include signatures of the youth and family. Senior Management and CJMs will not know if JCCs are conducting family sessions if there is no record of these sessions.

JCCs must be notified of their GRM ratings. The GRM is meant to provide feedback to the JCC on their FFPS skills sets. JCCs should be made aware in writing areas they need to improve and areas they have shown improvement.

The CJM workload increased. CJMs requested that their FFPS duties be prioritized. To accomplish this CJMs recommended that Multnomah County Department 
of Community Justice hold fewer meetings. CJMs indicated that issues and decisions covered in these meetings should be sent via email to the CJMs. Other recommendations included that the CJMs complete fewer paperwork required by the county and possibly promote another employee to a CJM position.

\section{Limitations}

There were several limitations to this study. First, at the time of the study not all of the JCCs at JSD were trained in FFPS. Many JCCs located in other departments (e.g. Youth Development Services) during FFPS training were not trained. This investigator was asked to not interview the non-trained JCCs. This investigator believes that nontrained JCCs delivering FFPS should have been included, for the success of FFPS's implementation depends on the entire organization. However, JSD management would not allow me to interview the non-trained JCCs because management did not understand the value of the non-trained JCCs' input and opinions on the implementation of FFPS. Second, the families and youth who received this case management model were not included in this study. Interviews of the youth and family would have given a better understanding of the model and provided critical information on their perceptions on FFPS being delivered by a JCC. It is to be noted too that interviews of the youth and family would have provided a more in-depth understanding if they found FFPS helpful. Unfortunately because this study needed to be completed by October, this investigator did not have the time to interview the youth and family. Third, observations of family FFPS sessions 
should have been included in the study. Many JCCs and CJMs expressed concern over their competency. Unfortunately, this investigator does not have enough FFPS experience to observe FFPS family sessions; nor does JSD contract an independent experienced evaluator. The literature on family-based interventions recommends that only experienced, independent FFT/FFPS trainers and counselors ascertain community corrections professional's model adherence and competence (Rowland, 2007; Sexton, 2011). This investigator only had been trained in FFPS and had attended and performed on a part time basis for one year.Fourth, data was not available to indicate the dosage and frequency the JCCs were delivering FFPS. Data collecting the number of times and the dosage of JCC delivery may have corroborated whether and how often the JCCs were delivering FFPS. JSD CJMs and Senior Managers were aware of this problem and recommended that a data collection system indicating FFPS dosage and frequency be integrated into the JJIS system. Fifth, because this was a process evaluation and not an outcome study, this did not compare FFPS and non-FFPS youth recidivism. In the future quantitative outcome studies may provide additional information on FFPS's efficacy in reducing recidivism. Sixth, many African American and Latino youth began receiving additional services through the Community Healing Initiative (CHI) during the time of the study. $\mathrm{CHI}$ is a family and community-centered collaboration program outside of JSD that provides culturally specific services to African American and Latino youth. The goal of CHI is to prevent high-risk youth of color from committing new crimes and from penetrating into the 
system further. Because these youth were receiving services in addition to FFPS it may be difficult to determine FFPS's efficacy. The JCCs perceptions that family functioning is increasing may be due to $\mathrm{CHI}$ or to other services youth are referred, such as substance treatment, sex offender treatment, and individual counseling services. Some of the JCCs self-reports perceived increased family functioning during FFPS family sessions and were unable to discern if increased family functioning was because Seventh, as Mashall and Rossman (2011) argue interviewers sometimes may tend to lie and not tell the truth about their own perceptions and perceived behaviors. Eighth, it must be noted in all qualitative research that the themes and results generated from this study are filtered by the biases and perceptions of this investigator. As a participant/observer during this case study, this investigator consulted with Portland State University sociology professors to keep this investigator as objective as possible. Finally, due to the nature of small sample sizes inherent to qualitative studies this study may not be generalizable to other juvenile justice agencies. Though the study is relevant and it can be reasonably said that this study may have possible applications to other juvenile justice organizations considering FFPS as a case management model.

\section{Recommendations for Future Research}

Based on the results of the study there are several recommendations for future research. First, interviews of the youth and parents will provide a better understanding of the JCC's model adherence and competence. Moreover, interviews of the youth and 
parents can provide information on if they think FFPS is effective and how they feel about a community corrections professional delivering FFPS to them. Second, past studies suggest a counselor's model adherence and competence moderates program outcomes (Barnoski, 2002, 2004, 2005; Rowland, 2007). Observations of family sessions and FFPS consultation sessions by an experienced, independent FFT/FFPS counselor would elucidate the JCCs and CJMs model adherence and competency. Rowland's (2007) study found that when both juvenile managers and independent FFT/FFPS trainers were conducting the GRM ratings of the juvenile parole officers in Washington, Rowland (2007) found that juvenile parole managers were more lenient than the independent, experienced FFT/FFPS trainers and counselors. Thirdly, future studies should include a quantitative outcome study examining youth recidivism. Because the Department of Community Justice will be providing FFPS to most youth and families, the youth and families will not be randomly assigned. It has been suggested that a time-series analysis compares the recidivism levels of matched medium and high-risk FFPS youth with medium and high-risk historical non-FFPS youth (Posavac \& Carey, 2007). A time-series design specifically for FFPS is a quasi-experimental research design in which monthly measurements would collect recidivism data on youth during the the time and after the youth and family receive the FFPS intervention. A time-series design is a way of meeting some of the internal validity challenges inherent to pre and post-test designs. Because maturation effects can be traced during the time periods before and after the intervention, 
the likelihood of confusing a program's effect with maturation effects is greatly reduced when using a time-series design rather than a pretest-postest design. 


\section{References}

Aarons, G. (2004). Mental health provider attitudes toward adoption of evidence-based practice: Evidence-based practice attitude scale (EBPAS). Mental Health Services Research, 4, 61-74.

Aarons, G., \& Sawitzky, A. (2006). Organizational culture and climate and mental health provider attitudes toward evidence-based practice. Psychological Services, 3, 6172.

Adler, P.A., \& Adler, P. (1998). Observational techniques. In Denzin, N. \& Lincoln, Y. (Eds.), Collecting and interpreting qualitative materials (pp. 79-109). London: Sage.

Alexander, J., Barton, C., Gordon, D., Grotpeter, J., Hansson, K., Harrison, R., Mears, S., Mihalic, S., Parson, B., \& Pugh, C. (1998). Functional family therapy: Blueprints for violence prevention, book three. Boulder, CO: Center for Study and Prevention of Violence, Institution of Behavioral Science, University of Colorado.

Retrieved December 14, 2012 from http://www.colorado.edu/cspv/blueprints/modelprograms.html.

Alexander, J., \& Parsons, B. (1973). Short-term behavioral intervention with delinquent families: Impact on family process and recidivism. Journal of Abnormal Psychology, 81, 219-225. 
Aos, S., Lee, S., Drake, E., Pennucci, A., Klima, T., Miller, M., Anderson, L., Mayfield, J., \& Baily, M. (2011). Return on investment: Evidence-based options to improve statewide outcomes. (Document No.: 11-07-1201). Olympia: WA. Washington State Institute for Public Policy.

Altschuler, D., \& Armstrong, T. (2002). Juvenile corrections and continuity of care in a community context: The evidence and promising directions. Federal Probation, 66, 72-78.

Atkinson, P., \& Hammersley, M. (1998). Ethnography and participant observation. In N.K. Denzin \& Y.S. Lincoln (Eds.), Strategies of qualitative inquiry (pp. 110136). Thousand Oaks, CA: Sage.

Backer, T. (2002). Finding the balance: Program fidelity and adaptation in substance abuse prevention. A state-of-the-art review. Washington, D.C.: U.S. Department of Health and Human Services, Substance Abuse and Mental Health Services Administration, Center for Substance Abuse Prevention. Retrieved December 2011 from http://www.samhsa.gov.

Bar-Gal, D., \& Schmid, H. (1992). Organizational change and development in human service organizations. New York: Hawthorne Press.

Barnoski, R. (2002). Washington state's implementation of functional family therapy for juvenile offenders: Preliminary findings. (Doc No. 02-08-1201). Olympia: WA.: Washington State Institute for Public Policy. Retrieved July 2011 
http://www.wsipp.wa.gov/pub.asp?docid=02-08-1201.

Barnoski, R. (2004). Outcome evaluation of Washington state's research-based programs for juvenile offenders. (Document No: 04-01-1201). Olympia: WA.:Washington State Institute for Public Policy. Retrieved November 2011 from http://www.wsipp.wa.gov/pub.asp?docid=04-01-1201.

Barnoski, R. (2005). Washington state's experience with research-based juvenile justice programs. [Powerpoint slides]. Presented at the Los Angeles County Juvenile Justice Conference.CA. Retrieved September 2011 from http://www.wsipp.wa.gov/pub.asp?docid=05-19-2005.

Bogdan, R., \& Biklen S. (1998). Qualitative research for education: An introduction to theory and methods (3rd ed.) Boston: Allyn and Bacon.

Bogdan, R., \& Biklen, S. (2003). Qualitative research in education: An introduction to theory and methods (4th ed.). Needham Heights, MA: Allyn \& Bacon.

Bonner, A. \& Tohurst, G. (2002). Insider-outsider perspectives of participant observation. Nurse Researcher, 9, 7-19.

Boyatzis, R. (1998). Transforming qualitative information: Thematic analysis and code development. Thousand Oaks, CA: Sage.

Braun, V., \& Clarke, V. (2006). Using thematic analysis in psychology. Qualitative Research in Psychology, 3, 77-101.

Burr, V. 1995: An introduction to social constructionism. New York, NY: Routledge. 
Byrne, J., Lurigio, A., \& Baird, C. (1989). The effectiveness of the new intensive supervision programs. Research in Corrections 2, 1-48.

Byrne, J., \& Kelly, L. (1989). Restructuring probation as an intermediate sanction:

Evaluation of the Massachusetts intensive probation supervision program.

Washington, DC: National Institute of Justice

Casey Family Programs. (July 2010). Stories of practice change in juvenile probation:

Using flexible funds in Los Angeles county to support youth and their families.

Retrieved December 2011 from

http://www.colorado.edu/cspv/blueprints/modelprograms.html.

Center for the Study and Prevention of Violence. (2012). Blueprints program guide.

Retrieved December 2012 from http://www.colorado.edu/cspv/.

Charmaz, K. (2000). Grounded theory: Objectivist and constructivist methods. In N.

Denzin \& Y. S. Lincoln (Eds.), Handbook of qualitative research (2nd ed.). Thousand Oaks, CA: Sage.

Charmaz, K. (2006). Constructing grounded theory: A practical guide through qualitative analysis. London: Sage Publications.

Clandinin, J., \& Connelly, M. (1998). Personal experience methods. In N. K. Denzin \& Y. S. Lincoln (Eds.), Collecting and interpreting qualitative materials (pp. 150-178). Thousand Oaks, CA: Sage.

Clear, T. \& Latessa, E. (1993). Probation officer roles in intensive supervision: 
Surveillance versus treatment. Justice Quarterly, 10, 441-462.

Cohen, L., \& Manion, L. (1994). Research methods in education (4th ed.) London: Routledge.

Cooke, R., \& Rousseau, D. (1988). Behavioral norms and expectations: A quantitative approach to the assessment of organizational climate. Group \& Organization Studies, 13, 245-273.

Cooke, R., \& Szumal, J. (1993). Measuring normative beliefs and shared behavioral expectations in organizations: The reliability and validity of the organizational culture inventory. Psychological Report, 72(3), 1299-1330.

Cooke, R., \& Szumal, J. (2000). Using the organizational culture inventory to understand the operating culture of organizations. In N.M. Ashkanasy \& C. Wilderom, Peterson, J. (Eds.), Handbook of organizational culture and climate (pp. 147-162). Thousand Oaks, CA: Sage.

Corbin, J., \& Strauss, A. (2007). Basics of qualitative research: Techniques and procedures for developing grounded theory (3rd ed.). Thousand Oaks, CA: Sage.

Crabtree, B (1999). Doing Qualitative Research. Newbury Park, CA: Sage.

Creswell, J. (1998). Qualitative inquiry and research design: Choosing among five traditions. Thousand Oaks, CA: Sage.

Creswell, J. (2003). Research design: Qualitative, quantitative, and mixed methods approaches (2nd ed.). Thousand Oaks, CA: Sage. 
Creswell, J., \& Clark, V. (2007). Designing and conducting mixed methods research. Thousand Oaks, CA: Sage.

Crosnoe, R., Erickson, K., \& Dornbusch, S. (2002). Protective functions of family relationships and school factors on the deviant behavior of adolescent boys and girls. Youth \& Society, 33, 515-544.

Crotty, M. (1998). The foundations of social research: Meaning and perspective in the research process. London: Sage.

Cullen, F., \& Gendreau, P. (2001). From nothing works to what works: Changing professional ideology in the $21^{\text {st }}$ Century. The Prison Journal, 81, 313-338.

Dane, A., \& Schneider, B. (1998). Program integrity in primary and early secondary prevention: Are implementation effects out of control? Clinical Psychology Review, 18, 23-45.

Denzin, N. (1989). Interpretive interactionism (2nd ed.). Thousand Oaks, CA: Sage.

Denzin, N. (1989b). The research act. Englewood Cliffs, NJ: Prentice-Hall.

Denzin, N., \& Lincoln, Y. (Eds.) (1994). Handbook for Qualitative Research. Thousand Oaks, CA: Sage.

Department of Community Justice Juvenile Services Division. (2011). Impact of Implementation of FFP in Yolo Co. Probation, CA. [PowerPoint slide]. Retrieved September 2011 from http://multnomah.granicus.com/MetaViewer.php? meta_id=13492\&view=\&showpdf $=1$. 
Dodge, K., Dishion, T., \& Lansford, J. (Eds.). (2006). Deviant peer influences in programs for youth. New York, NY: Guildford.

Duncan, T., Davey, M., \& Davey, A. (2011). Transporting functional family therapy to community-based programs. The Family Journal, 19, 41-46.

Durlak, J. (1997). Successful prevention programs for children and adolescents. New York, NY: Plenum.

Durlak, J., \& DuPre, E. (2008). Implementation matters: A review of research on the influence of implementation on program outcomes and the factors affecting implementation. American Journal of Community Psychology, 41, 327-350.

Elliott, D., \& Mihalic, S. (2004). Issues in disseminating and replicating effective prevention programs. Prevention Science, 5, 47-54.

Fagan, A. (1990). Treatment and reintegration of violent juvenile offenders: Experimental results. Justice Quarterly, 7, 233-263.

Fagan, A., \& Mihalic, S. (2003). Strategies for enhancing the adoption of school-based prevention programs: Lessons learned from the Blueprints for Violence Prevention Replications of the Life Skills Training Program. Journal of Community Psychology, 31, 235-253.

Farrell, J., Young, D., \& Taxman, F. (2011). Effects of organizational factors on use of juvenile supervision practices. Criminal Justice and Behavior, 38, 565-581.

Feldman, S. (1993). How organizational culture can affect innovation. In L. Hirschhorn 
\& C.K. E. Barnett (Eds.), The psychodynamics of organizations: Labor and social change (pp. 85-97). Philadelphia: Temple University Press.

FFT, Inc. (2012). FFT: Functional Family Therapy. Retrieved August 15, 2012 from http://www.fftinc.com/sites.html.

Fixsen, D., Maoom. S., Blase, K., Friedman, R., \& Wallace, F. (2005). Implementation research: A synthesis of the literature. Tampa, FL: University of South Florida, Louise De la Parte Florida Mental Health Institute, The National Implementation Research Network. Retrieved December 12, 2012, from http://nirn.fmhi.usf.edu/resoureces/publications/Monograph/pdf/monograph_full.p df.

Fulton, B., Latessa, E., Stichman, A., Travis, L., Corbett, R., \& Harris, M. (1997). A review of research for practitioners. Federal Probation, 61, 65-76.

Fulton, B., Stichman, A., Travis, L., \& Latessa, E. (1997). Moderating probation and parole officer attitudes to achieve desired outcomes. The Prison Journal, 77, 295312.

Functional Family Services Probation Manual (2011). Initial Probation Training. Seattle, WA: FFT Inc.

Gendrea, P. Goggin, C., \& Smith, P. (1999). The forgotten issue in effective correctional treatment: Program implementation. International Journal of Offender Therapy \& Comparative Criminology, 43(2), 180-187. 
Gendreau, P., Goggin, C., \& Smith, P. (2001). Implementation guidelines for correctional program in the "real world." In G. A. Bernfeld, D. P. Farrington \& A.W. Leschied. (Eds.), Offender Rehabilitation and Practice: Implementing and Evaluating Effective Programs (pp. 87-112). New York: John Wiley \& Sons, Ltd.

Glisson, C. (2002). The organizational context of children's mental health services. Clinical Child and Family Psychology Review, 5, 233-253.

Glisson, C. (2007). Assessing and changing organizational culture and climate for effective services. Research on Social Work Practice, 17(6), 736-747.

Glisson, C., \& Hemmelgarn, A. (1998). The effects of organizational climate and interorganizational coordination on the quality and outcomes of children's service systems. Child Abuse and Neglect, 22, 401-421.

Glisson, C., \& James, L. (2002). The cross-level effects of culture and climate in human service teams. Journal of Organizational Behavior, 23, 767-794.

Gordon, D., \& Arbuthnot, J. (1988). The use of paraprofessionals to deliver home-based family therapy to juvenile delinquents. Criminal Justice and Behavior, 15, 364378.

Gordon, D., Graves, K., \& Arbuthnot, J. (1995). The effect of functional family therapy for delinquents on adult criminal behavior. Criminal Justice and Behavior, 22, $60-73$.

Greene, J. (1998). Qualitative program evaluation. In Denzin, N. \& Lincoln, Y. (Eds.), 
Collecting and interpreting qualitative materials (pp. 372-399). London: Sage. Greenwood, P. (2008). Prevention and intervention programs for juvenile offenders. The Future of Children, 18, 185-210.

Hardyman, P. (1988). No frills: A study of probation resources, activities and outcome. (Doctoral dissertation). Rutgers University, New Jersey.

Hawkins, D., Catalano, R., \& Miller, J. (1992). Risk and protective factors for alcohol and other drug problems in adolescence and early adulthood: Implications for substance-abuse prevention. Psychological Bulletin, 112, 64-105.

Hedeker, D., \& Gibbons, R. (1996). MIXREG: A computer program for mixed-effects regression analysis with autocorrelated errors. Computer Methods and Programs in Biomedicine, 49, 229-252.

Hemmelgarn, A., Glisson, C., \& James, L. (2006). Organizational culture and climate: Implications for services and interventions research. Clinical Psychology: Science and Practice, 13, 73-89.

Henggeler, S., Melton, G., Brondino, M. , Scherer, D., \& Hanley, J. (1997).

Multisystemic therapy with violent and chronic juvenile offenders and their families: The role of treatment fidelity in successful dissemination. Journal of Consulting and Clinical Psychology, 65, 821-833.

Henggeler, S., Pickrel, S., \& Brondino, M. (1999). Multisystemic treatment of substance abusing and dependent delinquents: Outcomes, treatment fidelity, and 
transportability. Mental Health Services Research, 1, 171-184.

Henggeler, S., \& Schoenwald, S. (2011). Social policy report evidence-based interventions for juvenile offenders and juvenile justice policies that support them. Social Policy Report, 25, 1-35. Retrieved February 2011 from http://mstservices.com/files/SPR.pdf.

Huey, S., Henggler, S., Brondino, M., \& Pickrel, S. (2000). Mechanics of change in multisystemic therapy: Reducing delinquent behavior through therapists adherence and improved family and peer functioning. Journal of Consulting and Clinical Psychology, 68, 451-467.

Jessor, R., Van Den Bos, J., Vanderryn, J., Costa, F., \& Turbin, M. (1995). Protective factors in adolescent problem behavior: Moderator effects and developmental change. Developmental Psychology, 31, 923-933.

Jones, R., Jimmieson, N., \& Griffiths, A. (2005). The impact of organizational culture and reshaping capabilities on change implementation success: the mediating role of readiness for change, Journal of Management Studies, 42: 361-386.

Joplin, J., Bogue, B., Campbell, N., Carey, M., Clawson, E., Faust, D., Florio, K., Wasson, B., \& Woodward, W. (Eds). (2004). Using an integrated model to implement evidence-based practices in corrections. What works and why: Effective approaches to reentry (pp. 109-146). Lanham, MD: Author. Juvenile Rehabilitation Administration. (2008). Intensive parole model for high-risk 
juveniles offenders. Retrieved July 2010 from

http://www.dshs.wa.gov/pdf/main/legrep/Leg1208/IntensiveParoleModelforHighRiskJuvOffenders.pdf

Juvenile Rehabilitation Administration. (2012). Intensive parole model for high-risk juvenile offenders. Retrieved July 2010 from http://www.dshs.wa.gov/pdf/main/legrep/Leg0912/Intenisve\%20Parole \%20Legislative\%20Report\%20-\%202012.pdf

Kazdin, A. (2001b). Psychosocial treatments for conduct disorder in children and adolescents. In P.E. Nathan \& J.M. Gorman (Eds.), A guide to treatments that work. (pp. 57-85). New York, NY: Oxford University Press.

Kazdin, A., Mazurick, J., \& Bass, D. (1993). Risk for attrition in treatment of antisocial children and families. Journal of Clinical Child Psychology, 22, 2-16.

Klein, K., \& Forehand, R. (1997). Delinquency during the transition to early adulthood: Family and parenting predictors from early adolescence. Adolescence, 32, 61-81. Kogan, L. (1980). A family systems perspective on status offenders. Juvenile \& Family Court Journal, 31, 49-53.

Latessa, E. (2004). The challenge of change: Correctional programs and evidence-based practices. Criminology \& Public Policy 3, 547-560.

Latessa, E., \& Holsinger, A. (1998). The importance of evaluating correctional programs. Assessing outcome and quality. Corrections Management Quarterly, 2(4), 22-29. 
Lerch, J., Viglione, J., Eley, E., James-Andrews, S., \& Taxman, F. (2011). Organizational readiness in corrections. Retrieved August 2012 from http://www.uscourts.gov/uscourts/FederalCourts/PPS/Fedprob/201106/02 organizational.html

Lipsey, M. (1992). Juvenile delinquency treatment: A meta-analytic inquiry into the variability of effects. In T.D. Cook, H. Cooper, D.S. Cordray, H. Hartmann, L.V. Hedges, R.J. Light, T.A. Louis, \& F. Mosteller (Eds.), Meta-analysis for explanation. A casebook. (pp. 83-128). New York: Sage Publications.

Lipsey, M. (2009). The primary factors that characterize effective interventions with juvenile offenders: A meta-analytic overview. Victims and Offenders:

A International Journal of Evidence-based Research, Policy, and Practice, 4(2), 124-147.

Lipsey, M., Howell, J., Kelly, M., Chapman, G., \& Carver, D. (2010). Improving the effectiveness of juvenile justice programs: A new perspective on evidence-based practice.

Lipsey, M. \& Wilson, D. (1998). Effective interventions for serious juvenile offenders: A synthesis of resarch. In R. Loeber \& D.P. Farrington (Eds.), Serious and violent offenders: Risk factors and successful interventions. (pp. 313-345). Thousand Oaks, CA: Sage Publications, Inc.

Lowenkamp, C., Latessa, E., \& Smith, P. (2006). Does correctional program quality 
really matter? The impact of adhering to the principles of effective intervention. Criminology \& Public Policy, 5, 575-594.

Lynch, M. (1998). Waste managers? The new penology, crime fighting, and parole agency identity. Law \& Society Review, 32(4), 839-869.

Madden-Dirdich, D., Leonard, S., \& Gunnell, G. (2002). Parents' and childrens' perceptions of family processes and inner-city families with delinquent youth: A qualitative investigation. Journal of Marital and Family Therapy, 28, 355-369.

Marion, N., \& Oliver, W. (2006). The Public Police of Crime and Criminal Justice. Upper Saddle River, NJ: Prentice Hall.

McGuire, J. (2010). Comparing coercive and non-coercive interventions. Centre for Crime And Justice Studies. London: King's College. Retrieved February 3, 2012 from http://www.crimeandjustice.org.uk/opus1755/T2A coercive.pdf.

Marshall, C., \& Rossman, G. (2011). Designing Qualitative Research. London: Sage Publications.

McNeil, F., Burnett, R., \& McCulloch, T. (2010). Culture, Change, and Community Justice. The Scottish Centre for Crime and Research Report No. 02/2010. Retrieved January 2013 from http://www.sccjr.ac.uk/wp-content/uploads/2012/10/SCCJR\%20report \%20No_02:2010.pdf.

Merriam, S. B. (2009). Qualitative research: A guide to design and implementation. San 
Francisco: Jossey-Bass.

Mertens, D.M. (2005). Research methods in education and psychology: Integrating diversity with quantitative and qualitative approaches (2nd ed.). Thousand Oaks: Sage.

Mihalic, S. (2002). The importance of implementation fidelity. Retrieved January 2012 from http://incredibleyears.comwww.incredibleyears.com/Library/items/fidelityimportance.pdf.

Mihalic, S., \& Irwin, K. (2003). Blueprints for violence prevention: From research to real-world settings_-factors influencing the successful replication of model programs. Youth Violence and Juvenile Justice, 1, 307-329.

Mihalic, S., Irwin, K., Fagan, A., Ballard, D., \& Elliott, D. (2004). Successful program implementation: Lessons from blueprints. Washington, D.C.: Office of Juvenile Justice and Delinquency Prevention, Office of Justice Programs, U.S. Department of Justice. Retrieved December 2011 from https://www.ncjrs.gov/pdffiles1/ojidp/204273.pdf.

Mintzberg, H. (1993). Structures in fives: Designing effective organizations. Upper Saddle River: NJ: Prentice-Hall.

Mykota, D. (2008). Implementing paraprofessionals strength-based early intervention home visitations. Evaluation and Program Planning, 31, 266-276.

Onwuegbuzie, A. (2002). Why can't we all get along? Towards a framework for unifying 
research paradigms. Education. Retrieved June 2010, from http://findarticles.com/p/articles/mi_qa3673/is_/ai_n28923153.

Patton, M. (2002). Qualitative research and evaluation methods (3rd ed.). Thousand Oaks, CA: Sage.

Petersilia, J. (1990). Conditions that permit intensive supervision programs to survive. Crime and Delinquency, 36, 126-145.

Pollard, J., \& Hawkins, J. (1999). Risk and protection: Are both necessary to understand diverse behavioral outcomes in adolescence. Social Work Research, 23, 145-158.

Posavac, E., \& Carey, R. (2007). Program evaluation: methods and case studies. Upper Saddle River, NJ: Pearson Education, Inc.

Przybylski, R. (2008). What works: Effective recidivism reduction and risk-focused prevention programs. Retrieved October 10, 2011 from https://www.ncjrs.gov/App/abstractdb/AbstractDBDetails.aspx?id=245481.

Puzzanchera, C., Stahl, A., Finnegan, T., Tierney, N., \& Sunder, H. (2004). Juvenile court statistics 2000. Washington, D.C.: National Center for Juvenile Justice.

Puzzanchera, C., \& Kang, W. (2010). Easy access to juvenile court statistics: 1985-2007. Retrieved December 14, 2011 from http://ojjdp.ncjrs.gov/ojstatbb/ezajsc/.

Rhine, E., Mawhorr, T., \& Parks, E. (2006). Implementation: The bane of effective correctional programs. Criminology and Public Policy, 5, 347-358.

Rhule, D. (2005). Take care to do no harm: Harmful interventions for youth problem 
behavior. Professional Psychology: Research and Practice, 6, 618-625.

Rowland, M. (2007). Family-focused reintegration for youth on parole: Evaluation of a state-wide program. (Doctoral Dissertation). Retrieved from Proquest Database. (UMI No. 3274254).

Sampson, R., \& Laub, J. (1993). Crime in the making: Pathways and turning points through life. Cambridge, MA.: Harvard University Press.

Schein, E. (2004). Organizational culture and leadership. San Francisco, CA: JosseyBass.

Schoenwald, S., \& Hoagwood, K. (2001). Effectiveness, transportability, and dissemination of interventions: What matters when? Psychiatric Services, 5, 11901197.

Schwartz, M., \& Schwartz, C. (1955). Problems in participant observation. American Journal of Sociology, 60, 343-354.

Sexton, T., \& Alexander, J. (2002). Family-based empirically support interventions. The Counseling Psychologist, 30, 238-261.

Sexton, T., Alexander, J., \& Kopp, D. (2002). Functional Family Parole Training Manual FFP LLC.

Sexton, T. (2011). Functional family therapy in clinical practice: An evidence-based treatment model for working with troubled adolescents. London: Routledge, Taylor, \& Francis Group. 
Sexton, T., Ostrom, N., Bonomo, J., \& Alexander, J. (2000). Functional family therapy in a multicultural, multiethnic urban setting. Paper presented at the annual conference of the American Association of Marriage and Family Therapy, Denver, CO.

Shank, G. (2006). Qualitative research: A personal skills approach (2nd ed.). Upper Saddle River, NJ: Merrill Prentice Hall.

Sharma, M. (2004). Viable methods for evaluation of community-based rehabilitation programs. Disability and Rehabilitation. 26, 326-334.

Sickmund, M. (2006). Juvenile residential facility census, 2002: Selected findings. OJJDP National Report Series. Washington, D.C.: U.S. Department of Justice, Office of Juvenile Justice and Delinquency Prevention. Retrieved June 2010 from www.ojp.usdoj.gov/ojjdp.

Simon, J. (1993). Poor discipline: Parole and the social control of the underclass, 1890-1990. Chicago: University of Chicago Press.

Slayton, J., \& Llosa, L. (2005). The use of qualitative methods in large-scale evaluation: Improving the quality of the evaluation and the meaningfulness of the findings. Teachers College Record, 107, 2543-2565.

Smith, C., \& Stern, S. (1997). Delinquency and antisocial behavior: A review of family processes and intervention research. Social Service Review, 71, 382-420. 
Synder, H., \& Sickmund, M. (2006). Juvenile offenders and victims: 2006. National Report. U.S. Department of Justice. OJJDP. Retrieved June 2011 from http://www.ojjdp.gov/ojstatbb/nr2006/downloads/nr2006.pdf.

Szabla, D. (2007). A multidimensional view of resistance to organizational change: exploring cognitive, emotional, and intentional response to planned change across perceived change leadership strategies. Human Resource Development Quarterly $18,525-558$.

Tarolla, S., Wagner, E., Rabinowitz, J., \& Tubman, J. (2002). Understanding and treating juvenile offenders: A review of current knowledge and future directions. Aggression and Violent Behavior, 7, 125-143.

Ward, G., \& Kupchick, A. (2010). What drives juvenile probation officers?: Relating organizational contexts, status characteristics, and personal convictions to treatment and punishment orientations. Crime \& Delinquency, 56, 35-69.

White, T. (2005). Evidence-based practice in probation and parole: The implementation challenge. Perspectives, Summer.

Wilson, D. (1992). A strategy of change: Concepts and controversies in the management of change. London: Routledge.

Zazzali, J., Sherbourne, C., Hoagwood, K., Greene, D., Bigley, M., \& Sexton, T. (2008). The adoption and implementation of an evidence-based practice in child and family mental health services organizations: A pilot study of functional family 
therapy in New York State. Administration and Policy in Mental Health and Mental Health Services Research, 35, 38-49.

Yin, R. (2008). Case study research: Design and methods ( $4^{\text {th }}$ Ed.). Thousand Oaks, CA :Sage. 
Appendix A

\section{JUVENILE COURT COUNSELOR INTERVIEW QUESTIONS}

1. What is your educational background?

2. What is your understanding on why the department chose the Functional Family

Probation Services model instead of any other? Possible probe: How does this model impact your specific unit?

3. Functional Family Probation Services caseload size? Regular caseload size?

4. How do you think the youth and family are reacting to the delivery of this new model? Possible probe: The characteristics of the youth and family who are benefiting from Functional Family Probation Services? Those not benefiting? Probe: Do the youth and family like the service or is their resistance?

5. How do you use the Functional Family Probation Services Service Plan? Do you use the balanced alliance forms?

6. Tell me about your Functional Family Probation Services training? Probe: How did it help you? Not help? Probe: How change it?

7. What were you told about model adherence? Probe: Session length, dosage, how often meetings occur during the Engagement and Motivation Phase, during the Support \& Monitor, and Generalization phase.

8. Any safety issues out in the field?

9. What are Functional Family Probation Service's strengths and benefits? 
Appendix A (continued)

10. What are Functional Family Probation Service's weaknesses?

11. What are the barriers that preclude model adherence? Issues with implementation?

12. What are the facilitators that assist in model adherence? Issues with implementation?

13. What organizational culture and climate best suits Functional Family Probation

Services? Issues with implementation?

14. Do the Functional Family Probation Services Community Justice Manager consultation sessions ensure quality improvement? Probe: getting feedback, the GRM? 15. If you were to recommend this model to other agencies, why would you? Probe: Do you perceive it's working? You like or dislike it? 


\section{Appendix B \\ COMMUNITY JUSTICE MANAGER INTERVIEW QUESTIONS}

1. What is your understanding of your role in regards to Functional Family Probation Services? Probe: Any thoughts on the Functional Family Probation Services consultation meetings?

2. What are the quality controls and monitoring techniques used? Are they helpful?

3. How do you think Juvenile Court Counselors are progressing in the model? Probe: What has been helping them? Probe: What do you think they need more?

4. How are you using the global rating measure? Probe: How often? When?

5. What additional or needed support could you use as a manager? Any thoughts on implementation?

6. How has this new case management model changed your job?

7. How has this model changed the organizational culture and climate? Probe: structural changes?

8. Any barriers to program fidelity?

9. Any issues regarding the safety of JSD employees?

9. What are the facilitators within the organization to program fidelity?

10. Would you recommend this model to other juvenile justice jurisdictions? 


\section{Appendix C \\ SENIOR MANAGEMENT INTERVIEW QUESTIONS}

1. What is your understanding of your role in regards to Functional Family Probation Services? Probe: Any thoughts on the Functional Family Probation Services case staffings?

2. What is your understanding on why the department chose the Functional Family Probation Services model?

3. How has this model changed your job?

4. How has the culture and climate changed?

5. What are the quality controls and monitoring techniques used? Are they helpful?

6. How do you think the Juvenile Court Counselors and the Community Justice Managers progressing in the model? Probe: What has been helping them?

7. What do you think about safety?

8. Any barriers to program fidelity?

9. What are the facilitators within the organization to program fidelity?

10. What additional or needed support does JSD need to help with the changes?

11. Would you recommend this model to other juvenile justice jurisdictions? 
Appendix D

\section{FFPS CONSULTATION MEETING OBSERVATION CHECKLIST}

1. Name of unit and the number of Juvenile Court Counselors in attendance

2. Juvenile Court Counselors discussing Functional Family Probation Services phases (i.e. engagement and motivation)

3. Community Justice Manager discussing Functional Family Probation Services phases

4. Juvenile Court Counselors discussing Functional Family Probation Services skills (i.e. phases, reframing, balanced alliance, matching)

5. Community Justice Manager discussing Functional Family Probation Services

6. Indications of Global Rating Measure

7. Indications of Functional Family Probation Services case plans

8. Indications of Functional Family Probation Services tapes

9. Juvenile Court Counselors model adherence and competence

10. Community Justice Manager model adherence and competence

11. Indications of safety issues

12. Indications of technological issues

13. Indications of logistics (time, dosage)

14. Indications of Balance Alliance Questionnaires

15. Any indications of Community Justice Managers stating Functional Family Probation Services working or not working?

16. Any indications of Juvenile Court Counselors stating Functional Family Probation 


\section{Appendix D (continued)}

Services working or not working? Are there indications Juvenile Court Counselors are "giving up on a family?" or "keep trying?" Is there indications of burnout, cynicism, or dual role conflict? 
Research and Strategic Partnerships

Post Office Box 751 (RSP) Portland, Oregon 97207-075 rsp@pdx.edu
503-725-3423 tel 503-725-8170 fax

\section{S P Portland State}

\section{Portland State University HSRRC Memorandum}

To: Denise Austin

From: Mary Oschwald, Chair, HSRRC 2012

Date: May 2, 2012

Re: Your HSRRC application titled, "Bringing Functional Family Probation Services to the Community: A Mixed-Methods Approach" (HSRRC Proposal \#122118)

In accordance with your request, the Human Subjects Research Review Committee has reviewed your proposal referenced above for compliance with DHHS policies and regulations covering the protection of human subjects. The committee is satisfied that your provisions for protecting the rights and welfare of all subjects participating in the research are adequate, and your project is approved.

Please note the following requirements:

None

Changes to Protocol: Any changes in the proposed study, whether to procedures, survey instruments, consent forms or cover letters, must be outlined and submitted to the Chair of the HSRRC immediately. The proposed changes cannot be implemented before they have been reviewed and approved by the Committee.

Continuing Review: This approval will expire 05/02/2013, one year from the approval date. It is the investigator's responsibility to ensure that a Continuing Revien Report (available in ORSP) of the status of the project is submitted to the HSRRC approximately two months before the expiration date, and that approval of the study is kept current.

Adverse Reactions: If any adverse reactions occur as a result of this study, you are required to notify the Chair of the HSRRC immediately. If the problem is serious, approval may be withdrawn pending an investigation by the Committee.

Completion of Study: Please notify the Chair of the Human Subjects Research Review Committee (campus mail code ORSP) as soon as your research has been completed. Study records, including protocols and signed consent forms for each participant, must be kept by the investigator in a secure location for three years following completion of the study. 
April 18, 2013

If you have questions or concerns, please contact the HSRRC in the Office of Research and Strategic Partnerships, Market Center Building, Suite 620,1600 SW Fourth Ave, Portland OR 97207 (503) $725-2243$.

cc: Andrea Haack 


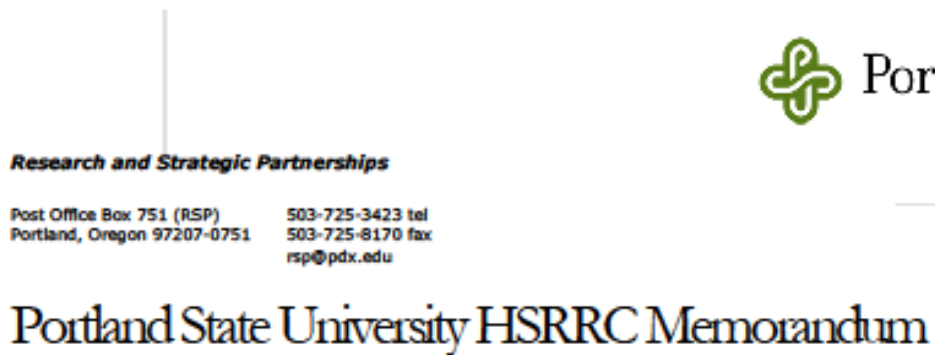

To: Denise Austin

From: Todd Bodner, HSRRC Chair, 2012

Date: July 25, 2012

Re: Approval of changes to your project entitled, "Bringing Functional Family Probation Services to the Community: A Mixed-Methods Approach" (HSRRC Proposal \#122118)

In response to your request for an approval of change in your original HSRRC application, the Human Subjects Research Review Committee has reviewred your above referenced project for compliance with Department of Health and Human Services policies and regulations on the protection of human subjects. The Committee is satisfied that your provisions for protecting the rights and welfare of all subjects participating in the research continue to satisfy federal requirements, and the changes are approved.

The following changes have been approved:

Changes submitted 7/18/2012: Addition of commnnity justice managers as interview subjects

Please be reminded that this project is due for continuing review two months before the expiration date of May 22, 2013. Please submit a continning review report at that time. The form is available at our website at http://www.pdx.edu/research/.

If you have questions or concerns, please contact the HSRRC in the Office of Research and Strategic Partnerships (RSP), (503) 725-2243, Market Center Building Suite 620, 1600 SW $4^{\text {th }}$ Ave, Portland OR 97207.

ec: Andrea Hanek 